\title{
Analysis of early steps in Assembly of Cytochrome $c$ Oxidase
}

\author{
Dissertation
}

for the award of the degree

"Doctor rerum naturalium"

Division of Mathematics and Natural Sciences of the Georg-August-Universität Göttingen

submitted by

Bettina Bareth

from Balingen

Göttingen 2013 

Member of the Thesis Committee (Reviewer):

Prof. Dr. Peter Rehling

Department of Cellular Biochemistry

Center for Biochemistry and Molecular Cell Biology

Georg-August-Universität Göttingen

Member of the Thesis Committee (Reviewer):

Prof. Dr. Stefan Jakobs

Mitochondrial Structure and Dynamics Group

Department of NanoBiophotonics

Max Planck Institute for Biophysical Chemistry, Göttingen

Member of the Thesis Committee:

Prof. Dr. Henning Urlaub

Bioanalytical Mass Spectrometry Group

Max Planck Institute for Biophysical Chemistry, Göttingen

Date of oral examination: 26.02.2014 
Affidavit:

I herewith declare that this thesis has been written independently and with no other sources and aids than quoted.

Bettina Bareth

Göttingen, 23.12.2013 


\section{LIST OF PUBLICATIONS}

Parts of this thesis have been published previously:

\section{Scientific research articles (peer-reviewed)}

The heme a synthase Cox15 associates with cytochrome $c$ oxidase assembly intermediates during Cox1 maturation.

Bareth B, Dennerlein S, Mick DU, Nikolov M, Urlaub H, Rehling P.

Mol Cell Biol. 2013 Oct; 33(20):4128-37.

Oxa1-ribosome complexes coordinate the assembly of cytochrome $c$ oxidase in mitochondria.

Keil M, Bareth B, Woellhaf MW, Peleh V, Prestele M, Rehling P, Herrmann JM.

J Biol Chem. 2012 Oct 5; 287(41):34484-93.

Mimicking a SURF1 allele reveals uncoupling of cytochrome $c$ oxidase assembly from translational regulation in yeast.

Reinhold R, Bareth B, Balleininger M, Wissel M, Rehling P, Mick DU.

Hum Mol Genet. 2011 Jun 15; 20(12):2379-93.

\section{Review article (non peer-reviewed)}

\section{Mitochondriale Logistik: Assemblierung der Cytochrom-c-Oxidase.}

Deckers M, Bareth B, Rehling P.

BioSpektrum. 08/2013; 19(4). 


\section{TABLE OF CONTENTS}

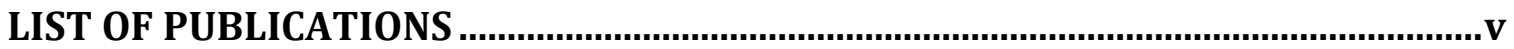

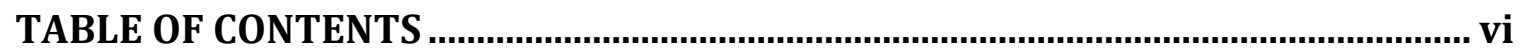

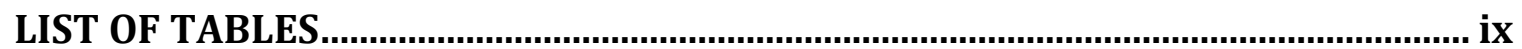

LIST OF FIGURES

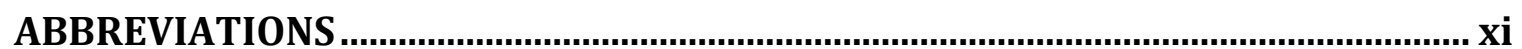

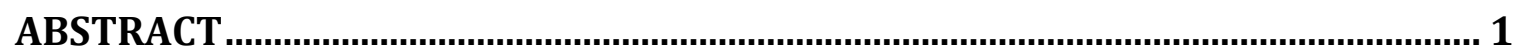

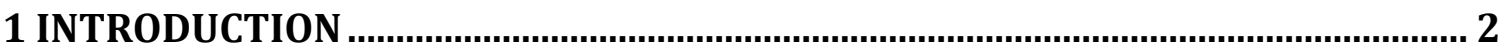

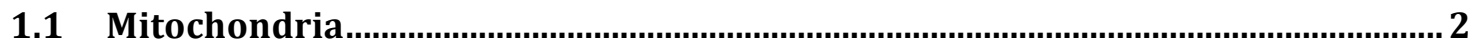

1.1.1 Origin, evolution and morphology of mitochondria ..................................................... 2

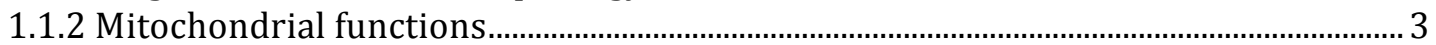

1.1.3 Oxidative Phosphorylation system ....................................................................................... 4

1.1.4 Mitochondrial genome/ dual genetic origin of mitochondrial proteome ..................... 7

1.1.5 Mitochondrial protein biogenesis..................................................................................... 9

1.1.5.1 Import pathways for nuclear-encoded proteins .......................................................................

1.1.5.2 Mitochondrial protein export ............................................................................................... 11

1.1.6 Mitochondria associated diseases ………….............................................................. 12

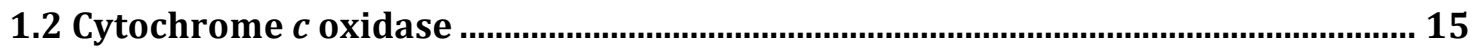

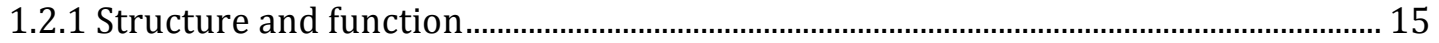

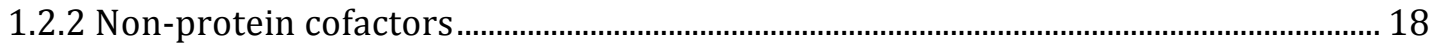

1.2.2.1 Heme $a$ biogenesis and insertion ......................................................................................... 18

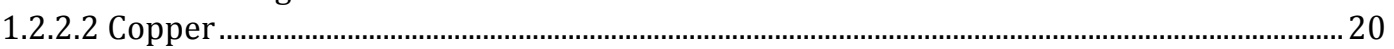

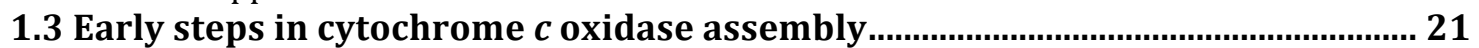

1.3.1. Membrane insertion of Cox1 by Oxa1 ....................................................................... 22

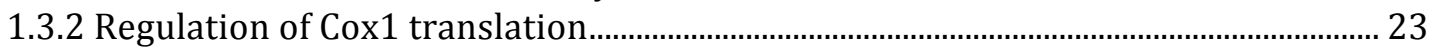

1.3.3 Coa3 and Cox14 stabilize newly synthesized Cox1 and regulate COX1 translation

1.3.4 Coa1 is required for progression of COX assembly ................................................... 24

1.3.5 Additional function of assembly factor Shy1 in heme transfer to Cox1?................... 25

1.3.6 Early COX assembly steps in higher eukaryotes ............................................................... 26

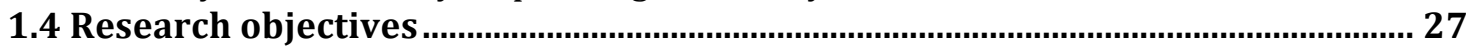

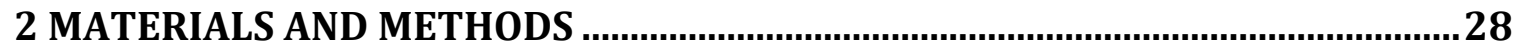

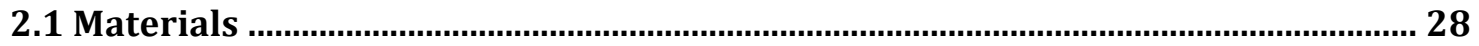

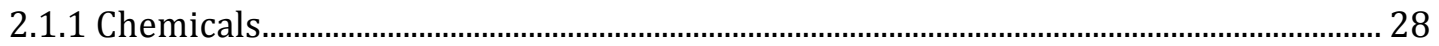

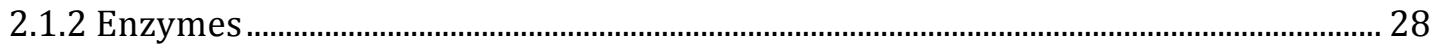

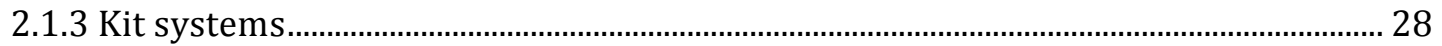

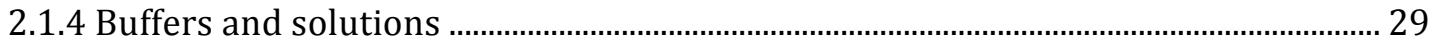

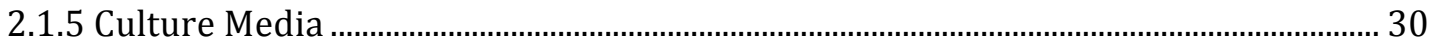

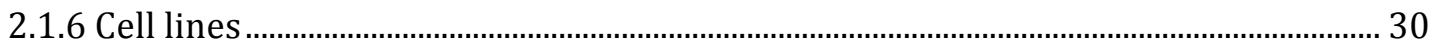

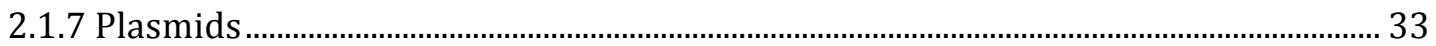

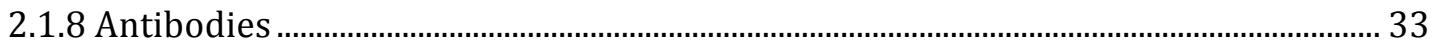

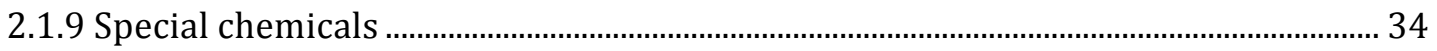




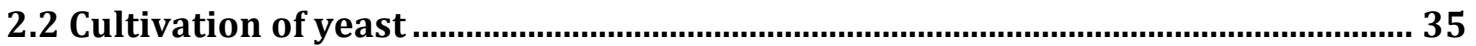

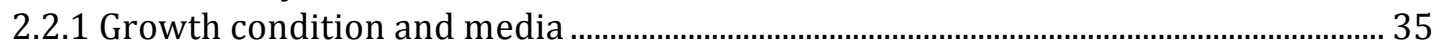

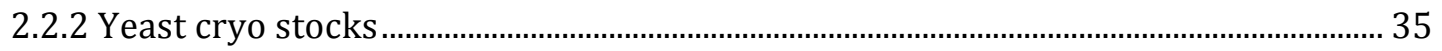

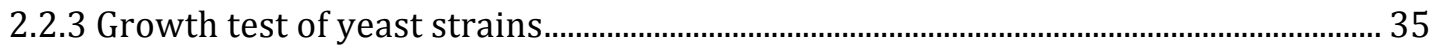

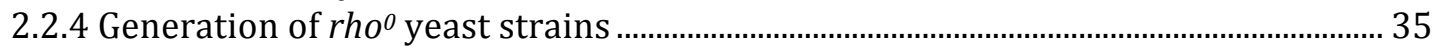

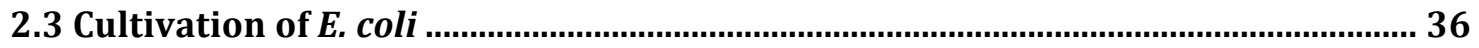

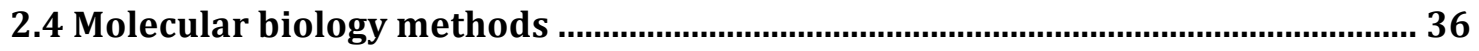

2.4.1 Amplification of DNA fragments by PCR....................................................................... 36

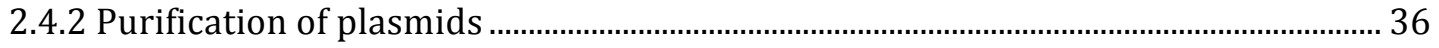

2.4.3 Preparation of yeast genomic DNA ……………............................................................. 37

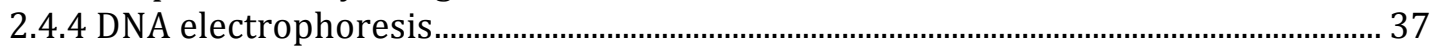

2.4.5 Determination of nucleic acid concentrations ……………………............................. 37

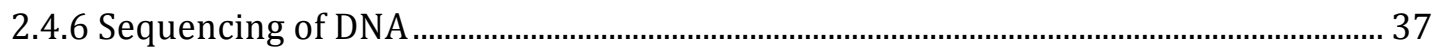

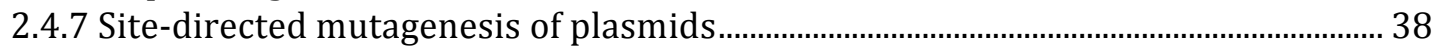

2.4.8 Chromosomal deletion and tagging of yeast genes ...................................................... 38

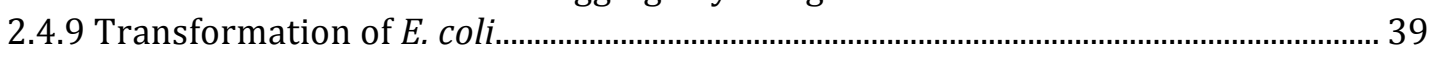

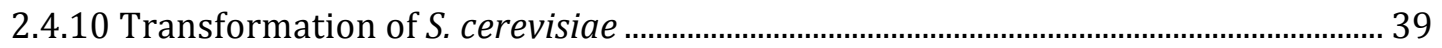

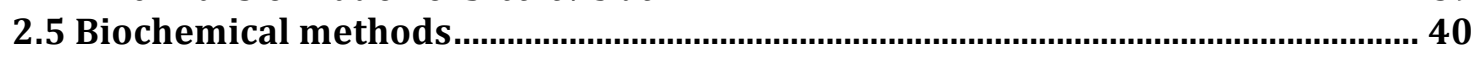

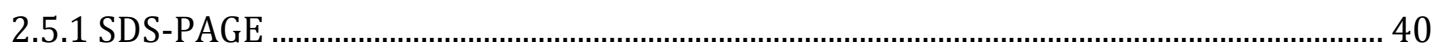

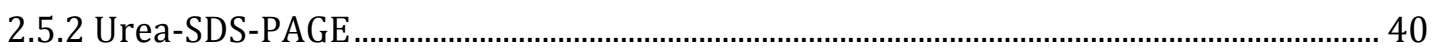

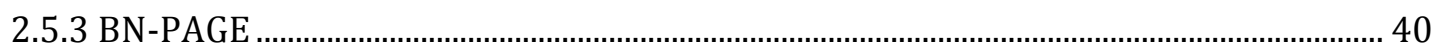

2.5.4 Determination of protein concentrations ....................................................................... 41

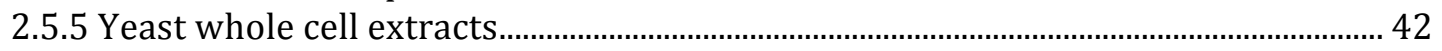

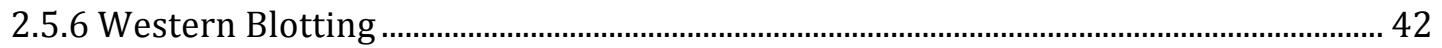

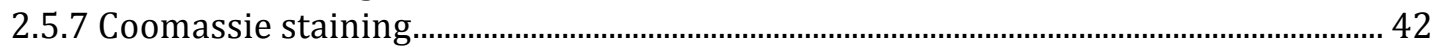

2.5.8 Immunodecoration of proteins on PVDF membranes …………………….................. 43

2.5.9 Detection of radiolabeled proteins by autoradiography ............................................... 43

2.5.10 Steady state protein analyses …………….................................................................. 43

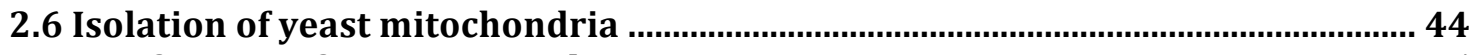

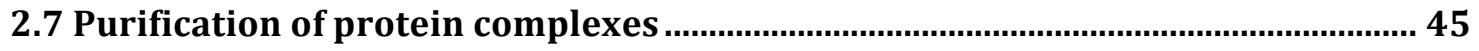

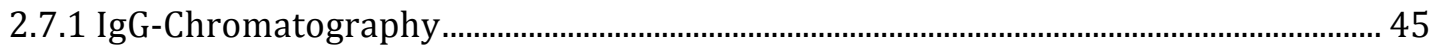

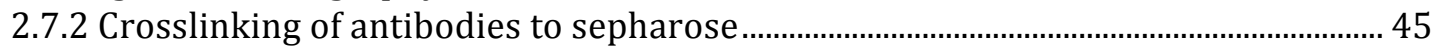

2.7.3 Co-immunoprecipitation .......................................................................................... 46

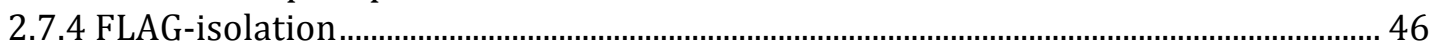

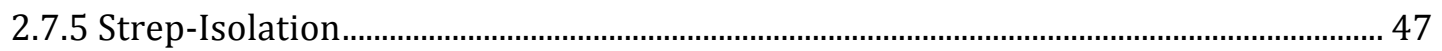

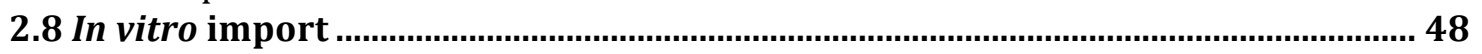

2.8.1 In vitro labeling of precursor proteins................................................................................ 48

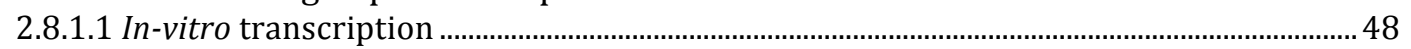

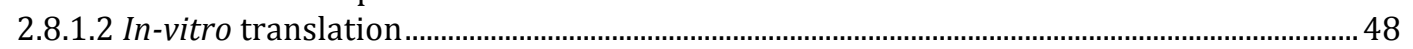

2.8.1.3 Coupled in vitro transcription/translation ................................................................................ 48

2.8.2 In vitro import into isolated mitochondria ................................................................... 49

2.9 Stable isotope labeling with amino acids in cell culture (SILAC) .............................49

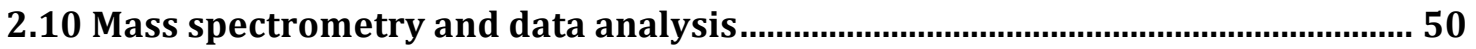

2.10.1 Mass spectrometry of native protein complexes ………………………………..... 50

2.10.2 Mass spectrometry of SILAC samples ………............................................................. 50

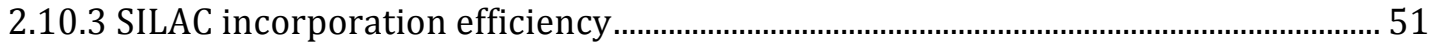


3 RESULTS

3.1 Translational regulation and early steps of COX assembly ..................................52

3.1.1 COX assembly in Oxa1 linker mutants.......................................................................... 52

3.1.1.1 Steady state protein levels are affected in Oxa1 linker mutants ..........................................52

3.1.1.2 Assembly of Cox5a/Cox13 is disturbed in 0xa1 mutants....................................................5 54

3.1.2 Isolation of new interaction partners of Mss51 ……………………........................... 56

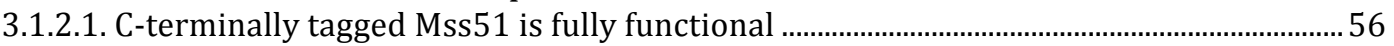

3.1.2.2 SILAC-labeling efficiency................................................................................................... 57

3.1.2.3 Interaction partners of Mss51 by SILAC ……………………………………….................. 58

3.1.2.4 Confirmation of SILAC results by Western Blot analysis ........................................................59

3.1.3 Nuclear-encoded subunits Cox5a and Cox6 in early COX assembly ...........................61

3.1.3.1 Import and assembly of radiolabeled Cox5a into COX assembly mutants.............................. 61

3.1.3.2 Requirement of Cox6 for recruitment of Shy1 to COA complexes ......................................... 63

3.1.4 Studying defects in COX assembly in Leigh syndrome: Characterization of a SHY1

point mutation (transferred from patient SURF1 mutation) ................................................. 65

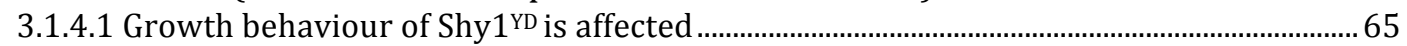

3.1.4.2 Accumulation of COA complexes in Shy1(YD)FLAG mitochondria...........................................67

3.2 Mapping Shy1-containing COA complexes: Association of heme $a$ synthase with Shy1

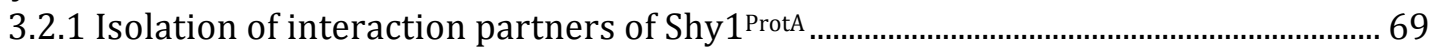

3.2.2 A C-terminal tag on Cox15 affects its association with COA complexes .................... 73

3.2.2.1 Growth behaviour of strains with C-terminally tagged Cox15 …………………………...... 73

3.2.2.2 COA components are not co-isolated with Cox15 ProtA .............................................................. 74

3.2.2.3 Co-Immunoprecipitation of Coa3 from wild type vs. Cox15 $5^{\mathrm{FLAG}}$..........................................75

3.2.3 COX assembly in strains with affected heme biogenesis ............................................... 76

3.2.3.1 Cox15 enzymatic function is not required for association to COA complexes .................. 76

3.2.3.2 Heme $o$ supply by Cox10 is not required for Cox15 interaction with COA complexes 80

3.2.4 Association of Cox15 and Shy1 with COA complexes .................................................... 82

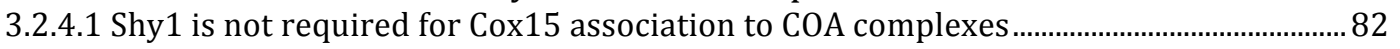

3.2.4.2 Cox15 is not required for Shy1 association to COA complexes............................................. 83

3.2.4.3 Cox15 associates with COA complexes in absence of Cox1 .................................................... 84

3.2.4.4 Cox15 and Shy1 form complexes in absence of mitochondrial DNA .................................. 85

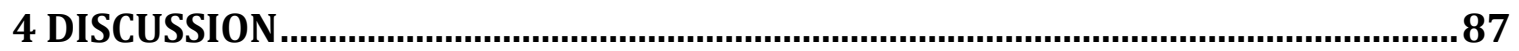

4.1 0xa1-Ribosome complexes coordinate the assembly of cytochrome $c$ oxidase 87

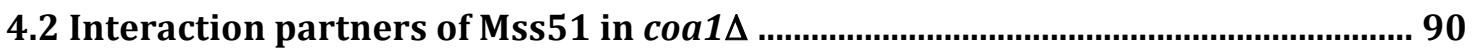

4.3 New order in the assembly process of COX: Cox5a and Cox6 .................................. 92

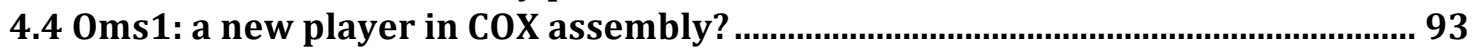

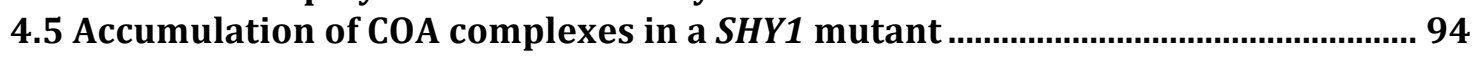

4.6 The heme $a$ synthase Cox15 associates with cytochrome $c$ oxidase assembly

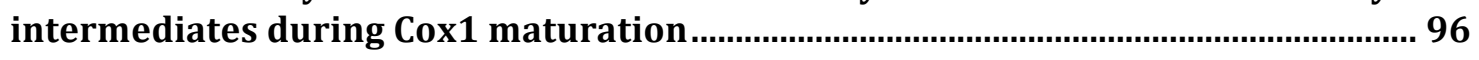

4.7 Association of Shy1 and Cox15 with COA complexes .............................................. 97

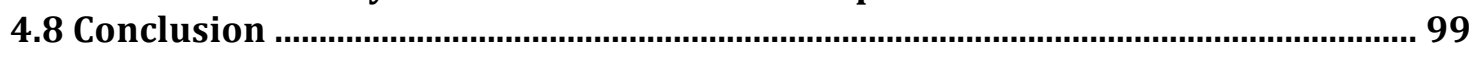

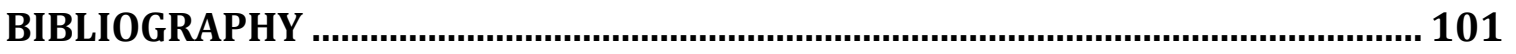

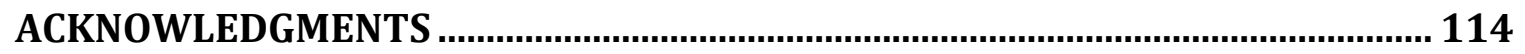




\section{LIST OF TABLES}

TABLE 1: Enzymes and suppliers.

TABLE 2: Kits used in this study...

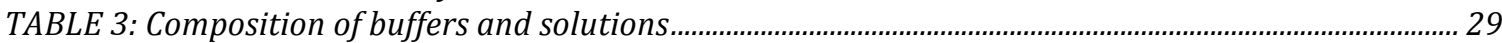

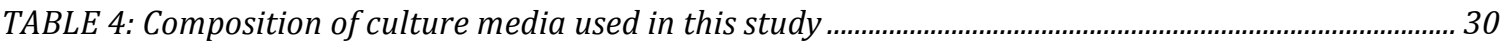

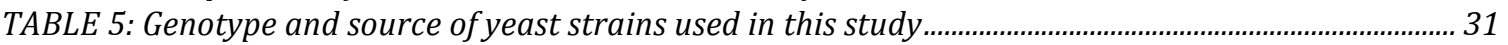

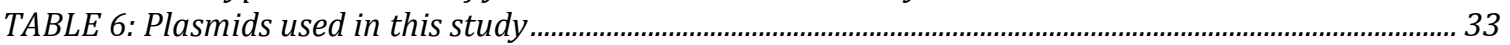

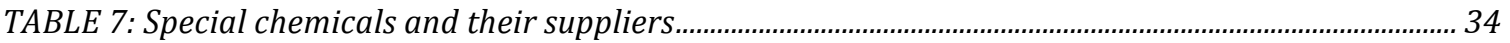




\section{LIST OF FIGURES}

FIG 1.1 Oxidative Phosphorylation system in mitochondria .......................................................................... 5

FIG 1.2 Biogenesis of respiratory chain complexes ............................................................................................ 8

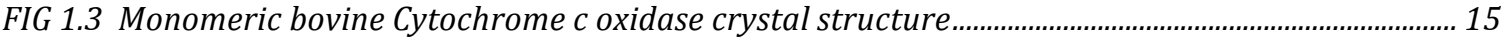

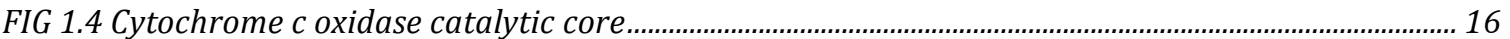

FIG 1.5 Biosynthesis of heme $o$ and heme a ............................................................................................... 18

FIG 1.6 Early steps of cytochrome c oxidase assembly and feedback regulation of Cox1 translation ........ 22

FIG 3.1 Analysis of steady state protein levels and OXPHOS complexes in Oxa1 linker mutants.................53

FIG 3.2 Import and assembly of radiolabeled Cox5a and Cox13 into Oxa1 linker mutants......................... 54

FIG 3.3 Analysis of growth behavior and steady state protein levels of strains with tagged Mss51 ...........56

FIG 3.4 Efficiency of incorporation of heavy amino acids into yeast proteins................................................. 57

FIG 3.5 Stable isotope labeling with amino acids in cell culture (SILAC)...................................................... 58

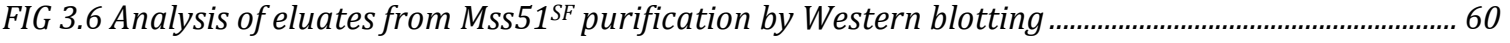

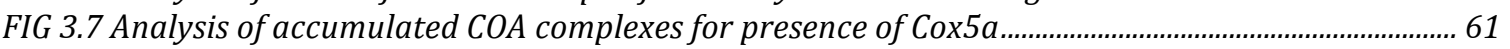

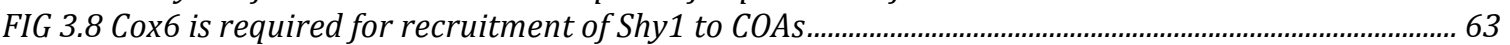

FIG 3.9 Analysis of growth behavior and steady state protein levels of strains with a Shy1 point mutation

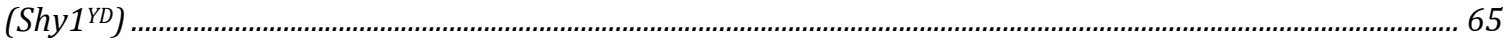

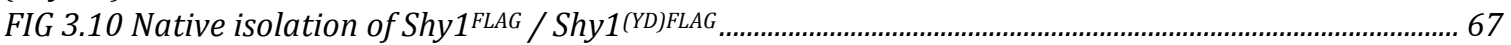

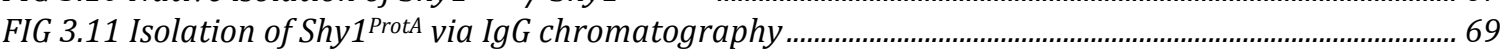

FIG 3.12 Mapping of Shy1 containing complexes by mass spectrometry ........................................................ 70

FIG 3.13 Mapping of Shy1 containing complexes by Western blotting ......................................................... 72

FIG 3.14 Analysis of growth behavior of strains with C-terminally tagged Cox15.................................... 73

FIG 3.15 Native isolation of Cox15 ProtA interaction partners via Ig G-chromatography.............................. 74

FIG 3.16 Influence of a C-terminal tag on Cox15 on interaction with COA complexes.................................. 75

FIG 3.17 Analysis of a Cox15 variant, defective in heme a biogenesis.............................................................. 76

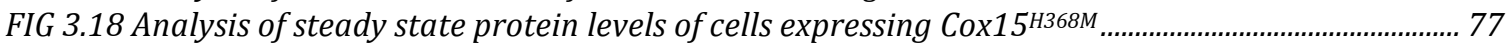

FIG 3.19 Association of Cox15 H368M with COA complexes........................................................................... 78

FIG 3.20 Composition of COA complexes in absence of heme o synthase (Cox10) or heme a synthase

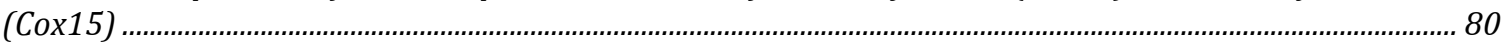

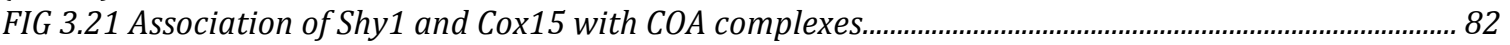

FIG 3.22 Association of Shy 1 and Cox15 with COA complexes in absence of Cox1 or Cox 2 .......................... 84

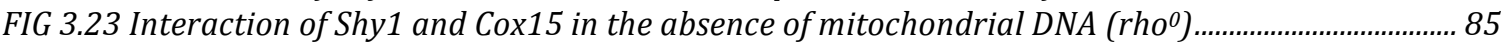

FIG 4.1 Mitochondrial ribosomes serving as a binding platform for COX assembly factors .........................89

FIG 4.2 The heme a synthase Cox15 forms complexes with Shy1 and associates with COA complexes...... 96

FIG 4.3 Interaction of Cox15 and Shy1 with COA complexes...................................................................... 98 


\section{ABBREVIATIONS}

ATP

BN

BSA

${ }^{\circ} \mathrm{C}$

CSM

DMP

DMSO

DNA

EDTA

FADH2

g

h

HCl

HRP

IgG

IM

IMS

kDa

l

LS

M

mA

mg

$\min$

ml

$\mathbf{m M}$

MOPS

mRNA

NADH

nm

OD

OM
Adenosine triphosphate

Blue Native

Bovine serum albumin

Degree Celsius

Complete supplement mixture

Dimethyl pimelimidate

Dimethyl sulfoxide

Deoxyribonucleic acid

Ethylenediaminetetraacetic acid

Flavin adenine dinucleotide

Gram

Hour(s)

Hydchloric acid

Horseradish peroxidase

Immunoglobulin G

(Mitochondrial) inner membrane

Intermembrane space

Kilodalton

Liter

Leigh syndrome

Mol per liter

Milliampere

Milligram

Minute(s)

Milliliter

Millimol per liter

3-(N-morpholino)propanesulfonic acid

Messenger ribonucleic acid

Nicotinamide adenine dinucleotide

Nanometer

Optical density/absorbance

(Mitochondrial) outer membrane 
PAGE

PBS

PCR

PMSF

PVDF

RNA

rpm

RT

SDS

sec

SF

SILAC

TBS

TCA

TIM

TOM

Tris

UTR

V

$\Delta \psi$

$\mu \mathbf{l}$

$\mu \mathrm{g}$

$\%$
Polyacrylamide gel electrophoresis

Phosphate buffered saline

Polymerase chain reaction

Phenylmethylsulfonyl fluoride

Polyvinylidene fluoride

Ribonucleic acid

Revolutions per minute

Room temperature

Sodium dodecyl sulfate

Second(s)

Streptavidin FLAG

Stable isotope labeling by/with amino acids in cell culture

Tris buffered saline

Trichloroacetic acid

Translocase of the inner membrane

Translocase of the outer membrane

Tris(hydroxymethyl)aminomethane

Untranslated region

Volt

Membrane potential

Microliter

Microgram

Percent 


\begin{abstract}
Cytochrome $c$ oxidase (COX) is the terminal enzyme of the respiratory chain. Due to its multimeric nature, assembly and maturation requires the concerted action of over 20 assembly factors in yeast. COX is composed of both nuclear- and mitochondria-encoded protein subunits. Its catalytic core is formed by three mitochondria-encoded subunits (Cox1, Cox2 and Cox3) harboring essential prosthetic groups. This study analysed different stages during early cytochrome $c$ oxidase assembly steps and extends the understanding of this process.

Upon separation of the ribosome-binding and membrane-insertion domains of Oxa1, defects in COX assembly were observed while membrane integration of mitochondrial translation products remained unaltered. These findings indicate that the Oxa1ribosome complex serves as a platform for binding of COX assembly factors and is therefore required for effective assembly of cytochrome $c$ oxidase.

By using SILAC analysis, Mss51 interaction partners from a complex, accumulating in a COX assembly mutant $(\operatorname{coa} 1 \Delta)$, were identified. This analysis demonstrated that the first nuclear-encoded subunits (Cox5a/Cox6) are added to assembly intermediates before the release of Mss51, suggesting a new sequence of COX assembly events. Additionally, the Mss51 interactome identified Oms1, a potential novel factor involved in COX assembly.

Interestingly, COX1 translational regulation is uncoupled from COX assembly in cells, expressing Shy1 ${ }^{\mathrm{YD}}$, a Leigh syndrome patient SURF1 mutation, transferred to yeast. I showed here that COX assembly intermediates accumulate in this mutant, explaining the mechanism behind the uncoupling phenomenon. In an unbiased characterization of Shy1-containing complexes, the heme $a$ synthase Cox15 was found to form complexes with Shy1. Furthermore, Cox15 associates with COX assembly intermediates, allowing insights into its role in the heme transfer mechanism during Cox1 maturation. Additional experiments suggested that Cox15 displays a role in COX assembly that is independent of its functions as the heme $a$ synthase.
\end{abstract}




\section{INTRODUCTION}

\subsection{Mitochondria}

\subsubsection{Origin, evolution and morphology of mitochondria}

The term "mitochondria" was first used in 1897, describing an intracellular structure that was first observed in the 1840s. According to the endosymbiont theory, mitochondria originate from the symbiosis of an aerobe bacterium ( $\alpha$-proteobacterium) (Sicheritz-Pontén et al. 1998; Gray et al. 2001) with an eukaryotic ancestor cell. The vast majority of the genetic information was transferred from the symbiont to the host nucleus during evolution (Adams \& Palmer 2003; Bowles et al. 2007), however the exact mechanism for this phenomenon remains unclear. As a consequence, mitochondria have to take up proteins, lipids and RNAs in order to fulfill their different biochemical functions in the cell. Thus, mitochondria were transformed from a symbiont to an organelle that got fully integrated into the cellular physiology (Dyall et al. 2004). Within cells, mitochondria form highly dynamic tubular networks that undergo permanent fusion and fission processes (Hoppins et al. 2007).

Mitochondria are enclosed by two phospholipid membranes with specific composition, resulting in four mitochondrial sub-compartments. The matrix is enclosed by the mitochondrial inner membrane and the intermembrane space separates the inner and outer membrane. Each compartment has features that allow them to carry out specialized functions. The outer mitochondrial membrane encloses the entire organelle and contains large numbers of integral proteins called porins that form channels allowing small molecules to freely diffuse from one side of the membrane to the other. Larger proteins are actively transported across the outer mitochondrial membrane by the translocase of the outer membrane (Endo \& Yamano 2010). For the exchange of e.g. lipids and calcium, the outer mitochondrial membrane can form contact sites with other organelles, such as the endoplasmic reticulum membrane (de Brito \& Scorrano 2010). 
Unlike the outer membrane, the inner membrane is highly impermeable to most molecules. In order to fulfill its functions, the mitochondrial inner membrane is extremely rich in proteins, harboring for example the oxidative phosphorylation system and the translocase of the inner membrane. Furthermore, this membrane is rich in the unusual phospholipid cardiolipin that is also found in bacterial plasma membranes (Hoch 1992). In order to increase the membrane surface and therefore the ability to produce energy, the inner membrane forms characteristic invaginations, called cristae (Frey et al. 2002). The matrix, enclosed by the inner membrane, contains metabolic enzymes but also the mitochondrial DNA and mitochondrial ribosomes.

Even though most genetic information of the endosymbiont was transferred to the nucleus, mitochondria maintained their own genome and translational machinery. The reason is speculated to be the high hydrophobicity of the encoded proteins which would complicate the transport across two membranes (Wallace 2007). The mitochondrial DNA is an extra-chromosomal element that is inherited in a non-mendelian, maternal fashion.

Due to the dual genetic origin of mitochondrial proteins, coordination of the mitochondrial protein expression and import during biogenesis is essential to maintain a functional organelle.

\subsubsection{Mitochondrial functions}

Mitochondria are best known for the production of ATP and therefore are often described by the popular term "powerplant of the cell". Thus, mitochondria carry out oxidative phosphorylation, the controlled burning of nutrients, coupled to the synthesis of ATP with its high-energy phosphate bonds (Mitchell \& Moyle 1968) that is used by the majority of cellular processes. In addition to their central role in ATP synthesis, mitochondria fulfill a number of other vital cellular functions. Central metabolic pathways, like the citric acid cycle and the $\beta$-oxidation of fatty acids are accommodated in mitochondria. Besides these functions, mitochondria also provide the cell with a number of metabolites such as amino acids and steroids. 
In contrast to the general believe, the ATP production by oxidative phosphorylation is not essential in many organisms; however, iron-sulfur cluster biogenesis in mitochondria is an essential process for all life even in non-respiring organisms (Kispal et al. 2005). In many organisms the respiratory function of mitochondria is dispensable or has been lost, e.g. the yeast S. cerevisiae is able to ferment sugar to produce ATP. Ironsulfur clusters are essential co-factors for a variety of cytosolic enzymes but also for mitochondrial proteins, involved in electron-transfer processes (Lill 2009).

Mitochondria also execute functions related to cell death in higher eukaryotes (Martinou \& Youle 2011), calcium signaling and storage (Rimessi et al. 2008) and are also the main source of reactive oxygen species (ROS) as inevitable by-products of cellular respiration (Pan 2011).

Due to central roles of mitochondria in energy production, reactive oxygen species biology, apoptosis and other cellular processes, their dysfunction is implicated with a diverse set of human mitochondrial disorders and diseases (see section 1.1.6).

\subsubsection{Oxidative Phosphorylation system}

Mitochondria oxidize nutrients via oxidative phosphorylation (OXPHOS) in order to generate energy. The mitochondrial OXPHOS system consists of two sub-systems, the electron transport chain (ETC), composed of complexes I-IV, and the ATP synthase (or complex V). The respiratory chain transfers electrons from reducing equivalents $\left(\mathrm{NADH}, \mathrm{FADH}_{2}\right)$, produced by e.g. the citric acid cycle within mitochondria, to molecular oxygen. The electron flux is coupled to proton pumping and therefore generates a proton gradient across the inner mitochondrial membrane $(\Delta \psi)$ (Hosler et al. 2006). This gradient drives the ATP synthesis via the $\mathrm{F}_{1} \mathrm{~F}_{0}$-ATP-synthase (Mitchell \& Moyle 1968; Yoshida et al. 2001) (FIG 1.1). 


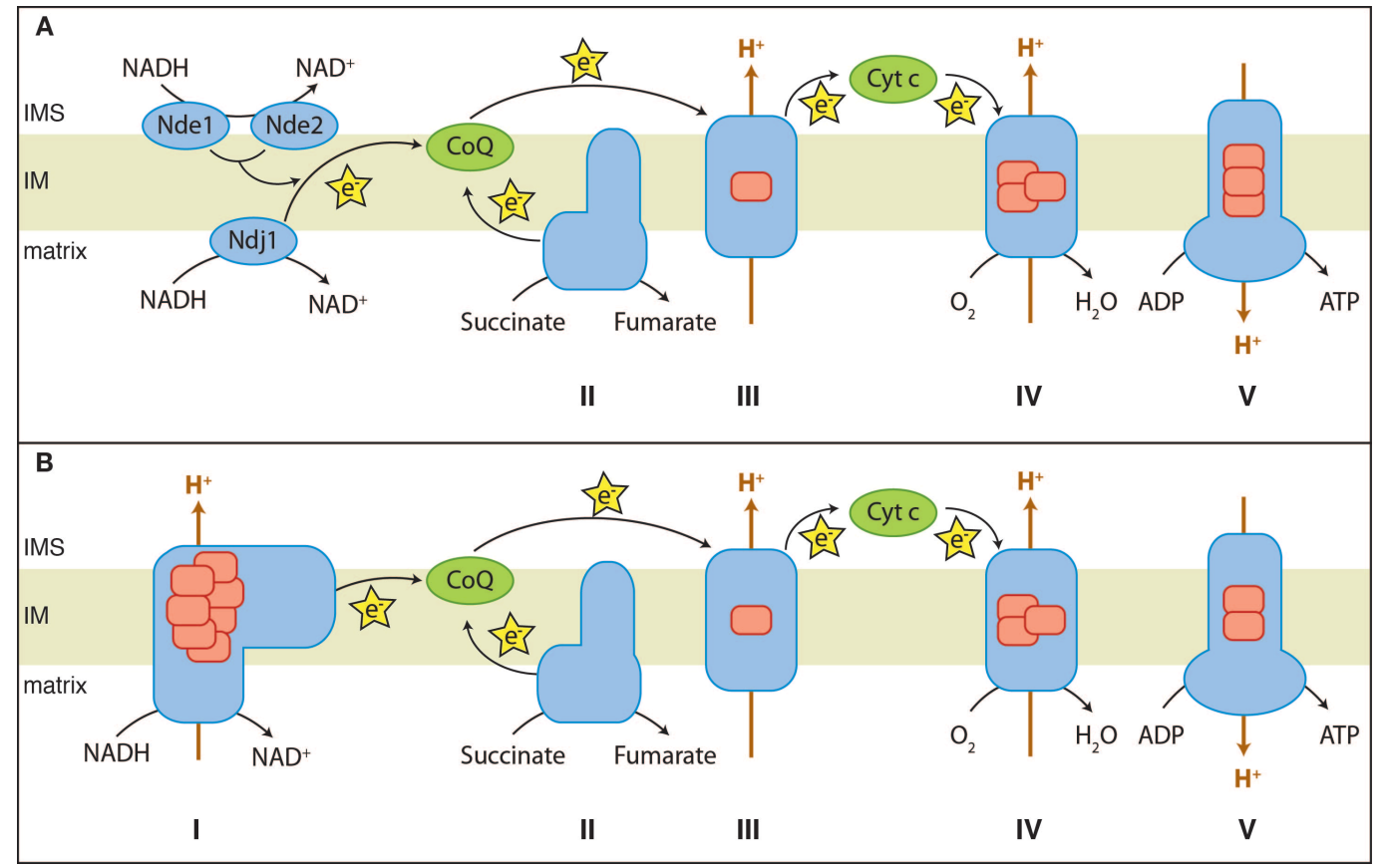

FIG 1.1 Oxidative Phosphorylation system in mitochondria

(A) Shown are the respiratory chain complexes II-IV and the $\mathrm{F}_{1} \mathrm{~F}_{0}$-ATP synthase (V) in the inner mitochondrial membrane of $S$. cerevisiae mitochondria (B) Respiratory chain complexes I-IV and the F1 Fo-ATP synthase (V) in the inner mitochondrial membrane of mammalian mitochondria. Electron flux (e) along the respiratory chain is illustrated. Brown arrows indicate proton $\left(\mathrm{H}^{+}\right)$flux across the inner membrane. Coenzyme $\mathrm{Q}(\mathrm{CoQ})$, cytochrome $c$ (Cyt c), intermembrane space (IMS). Mitochondria-encoded subunits are depicted in red

In detail, electrons are passed from the NADH dehydrogenase (complex I) and the succinate dehydrogenase (complex II) to coenzyme Q (CoQ). Coenzyme Q shuttles the electrons further to the cytochrome $b c_{1}$ complex (or cytochrome $c$ reductase; complex III). Subsequently, electrons are transferred to the mobile carrier cytochrome $c$ and to the cytochrome $c$ oxidase (complex IV). This terminal oxidase uses the electrons to reduce molecular oxygen to water. All OXPHOS complexes are multi-subunit complexes that are, with the exception of complex II, composed of mitochondrial- and nuclearencoded subunits.

Despite the functional conservation of the mitochondrial oxidative phosphorylation system, the composition of the complexes varies between species. FIG 1.1 illustrates the S. cerevisiae (A) and the mammalian (B) OXPHOS systems and their differences. In S. cerevisiae, no complex I but small proteins, fulfilling a similar function in electron transfer to CoQ, are found (Grandier-Vazeille et al. 2001). However, in contrast to the mammalian system, these proteins do not contribute to the proton gradient across the inner mitochondrial membrane. The yeast and the mammalian OXPHOS complexes also show differences in the total number of subunits and the number of mitochondriaencoded subunits (compare FIG 1.2). 
Although respiratory chain complexes II, III and IV have been crystallized as individual complexes, they can be visualized by BN-PAGE after mild solubilization of mitochondrial membranes as higher homo- and heterooligomers, called supercomplexes or respirasomes (Schägger \& Pfeiffer 2000; M. Zhang et al. 2002). It is however still under debate whether respiratory chain complexes are free moving entities, linked by the mobile electron carriers (fluid model) (Hackenbrock et al. 1986) or if they are indeed organized in supercomplexes (solid model) (Schägger \& Pfeiffer 2000). The solid model is challenged by the lack of evidence for the existence of supercomplexes in vivo. Furthermore the functional role of supercomplexes is not clear. The rational behind the formation of these supercomplexes is believed to be the optimization of electron transport and the reduction of distances for the mobile electron carriers between the complexes (Acín-Pérez et al. 2008). Using BN-PAGE analysis of solubilized yeast mitochondria, complex III is found as a dimer $\left(\mathrm{III}_{2}\right)$ as well as in complex with one or two copies of complex IV ( $\left.\mathrm{III}_{2} \mathrm{IV}, \mathrm{III}_{2} \mathrm{IV}_{2}\right)$. In mammals, complex III dimers associate with complex I and complex IV in various supercomplexes. The $\mathrm{F}_{1} \mathrm{~F}_{0}$-ATP-sythase (complex V) does not form hetero-oligomers and is found in its monomeric and (homo-)dimeric form ( $\mathrm{V}, \mathrm{V}_{2}$ ) (Arnold et al. 1998). The dimeric form of complex $\mathrm{V}$ is formed at a fixed angle and leads to membrane curvature (Paumard et al. 2002; Zick et al. 2009).

A recent study in mouse fibroblasts suggests that the organization into higher supercomplexes defines dedicated CoQ and cytochrome $c$ pools and that supercomplex assembly is dynamic and organizes electron flux to optimize the use of available substrates (Lapuente-Brun et al. 2013). The same study suggests that the solid and fluid state are just the very extremes of an equilibrium that the cell uses to adapt to varying carbon sources.

Supercomplex assembly factors are a matter of recent research. The yeast proteins Rcf1 and Rcf2 are proposed to be supercomplex III:IV assembly factors (Vukotic et al. 2012; Y.-C. Chen et al. 2012; Strogolova et al. 2012) In mammals, a factor required for the correct assembly of supercomplexes (SCAF1, originally termed Cox7A21), was recently identified (Lapuente-Brun et al. 2013). 


\subsubsection{Mitochondrial genome/ dual genetic origin of mitochondrial proteome}

Prior to the symbiosis of the proto-mitochondrion and the host cell, both had genomes sufficient for their independent lifestyles (Wallace 2007). However, upon transfer of genes to the nucleus, mitochondria were no longer independent but kept the core machineries for their biogenesis: Mitochondria retained their own genome, encoding for tRNAs, rRNA and 8 proteins in yeast (13 proteins in human). The proteins encoded by the $m t$ DNA are mainly the hydrophobic subunits of the respiratory chain complexes. In most species the mitochondrial genome is a circular DNA molecule, containing very compact genetic information. The mitochondrial DNA is inherited in a non-mendelian, maternal fashion. In humans and animals this is achieved, in part, by the dilution of the sperm $m t$ DNAs by ovarian $m t$ DNAs at fertilization, the oocyte having over 100,000 $m t$ DNA copies whereas the sperm only has hundreds (X. Chen et al. 1995) (residual paternal $m t$ DNAs are selectively removed in animals).

Although the genes encoded by the mitochondrial genome vary between species for not yet fully understood reasons, there is a minimal set of proteins that is mitochondriaencoded in all eukaryotes: All $m t$ DNAs analysed to date contain $C O B$ and $C O X 1$ genes, which are central to coupling electron transport to proton pumping in complexes III and IV, respectively (Wallace 2007).

In the yeast Saccharomyces cerevisiae, eight proteins are synthesized in the mitochondrial matrix: cytochrome $b$ (Cob) of the $b c_{1}$ complex; subunits 1-3 (Cox1, Cox2, and Cox3) of cytochrome $c$ oxidase; Atp6, Atp8, and Atp9 of the $\mathrm{F}_{1} \mathrm{~F}_{0}$-ATP-synthase; and the ribosomal protein Var1. In order to express these proteins, mitochondria retained a complete expression machinery.

Mitochondria contain around 1000 mitochondrial proteins in yeast (Sickmann et al. 2003; Reinders et al. 2006; Premsler et al. 2009). 99\% of these proteins are of cytosolic origin, translated at cytosolic ribosomes as precursor forms and subsequently imported into mitochondria. In contrast, the mitochondria-encoded subunits are translated on membrane-bound mitochondrial ribosomes and inserted into the inner mitochondrial membrane in a co-translational manner by the Oxa1 machinery (FIG 1.2). The number of nuclear and mitochondria-encoded subunits of the OXPHOS complexes (of mammals and yeast) are depicted in FIG 1.2. The dual genetic origin of mitochondrial proteins requires a coordination of the mitochondrial protein expression and import during organellar biogenesis. 


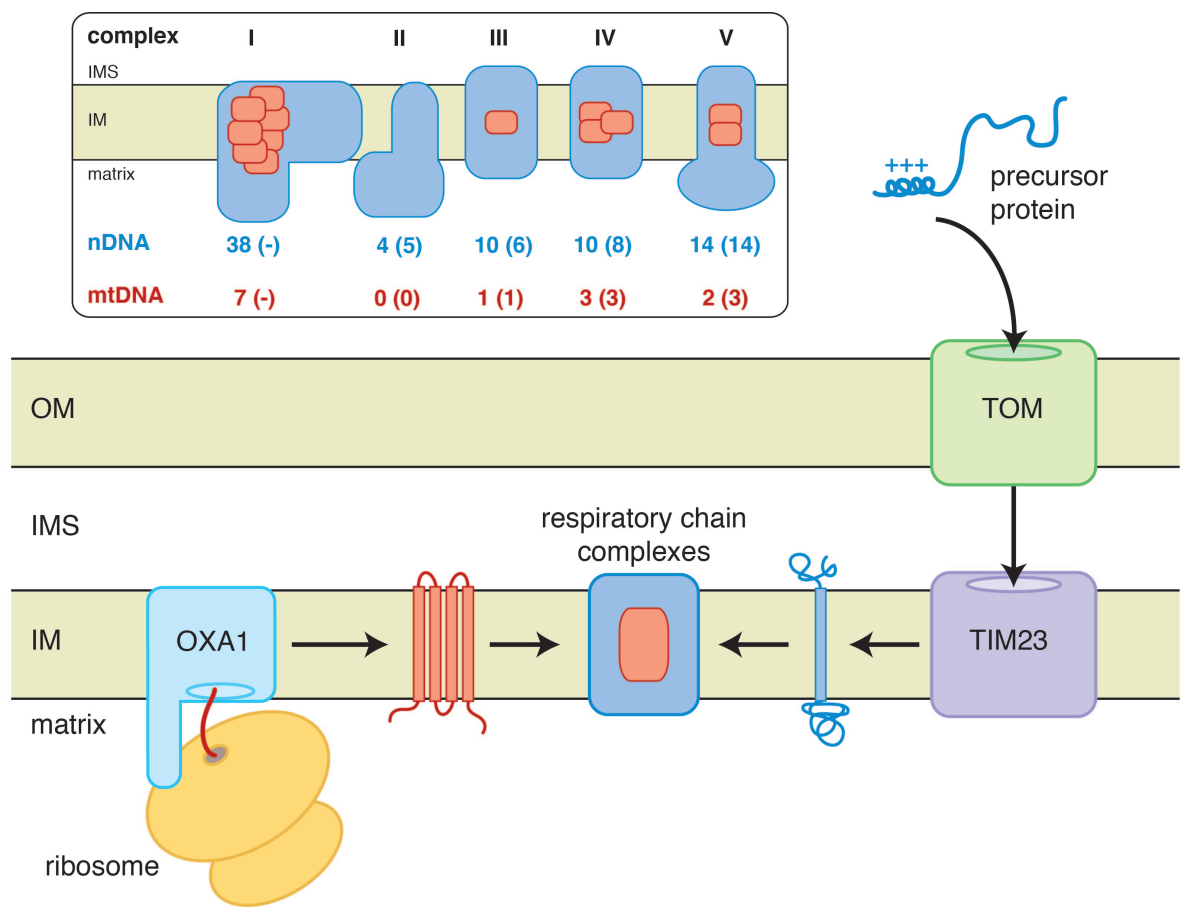

\section{FIG 1.2 Biogenesis of respiratory chain complexes}

Respiratory chain complexes are composed of subunits of dual genetic origin. Number of subunits per complex, encoded by the mitochondrial genome (mtDNA) are depicted in red, number of subunits, encoded by the nuclear genome (nDNA) in blue. Numbers are indicated for mammalian OXPHOS complexes (for S. cerevisiae in brackets). Cytosolic precursor proteins (blue) contain information that targets them to mitochondria (+++). Precursors are directed to the inner mitochondrial membrane by the translocase of the outer membrane (TOM) and the presequence translocase of the inner membrane (TIM23) complexes. Mitochondria-encoded proteins (red) are inserted into the inner mitochondrial membrane in a co-translational manner by the translocase oxidase assembly (Oxa1) machinery and assemble together with imported proteins into mature respiratory chain complexes. Outer membrane (OM), intermembrane space (IMS), inner membrane (IM) 


\subsubsection{Mitochondrial protein biogenesis}

\subsubsection{Import pathways for nuclear-encoded proteins}

Following translation on cytosolic ribosomes, precursor proteins are bound by cytosolic chaperones (Young et al. 2003) and imported into mitochondria along various pathways (Becker et al. 2012). It is widely accepted that import of precursor proteins into mitochondria occurs in a post-translational manner.

In general, precursor proteins are transported across or into the outer membrane by the TOM complex (Hill et al. 1998; Künkele et al. 1998; Model et al. 2008). After traversing the outer membrane, transport mechanisms diverge, depending on the targeting information present in the precursor proteins. A precursor protein is further transported to its destination (outer membrane, IMS, inner membrane or matrix) by specialized import machineries (Neupert \& Herrmann 2007; Chacinska et al. 2009; Dudek et al. 2013).

The most prevalent mitochondrial import signal is an $\mathrm{N}$-terminal extension termed presequence that is present in about $70 \%$ of mitochondrial precursors (Vögtle et al. 2009) and forms an amphipathic $\alpha$-helix with a net positive charge (Heijne 1986). Presequence-containing proteins are inserted into the inner membrane or imported into the matrix by the translocase of the inner membrane 23 (TIM23) (Becker et al. 2012; Dudek et al. 2013).

Complete matrix translocation of pre-proteins depends both on $\Delta \psi$ as well as on the additional activity of the presequence translocase-associated import motor, PAM (reviewed by Van Der Laan et al. 2010). Most N-terminal presequences are proteolytically removed after import by the mitochondrial processing peptidase and other proteases, resulting in an N-terminal truncation of the mature protein. (Taylor et al. 2001; Mossmann et al. 2012). However, some proteins contain presequences that are followed by a hydrophobic sorting signal. This sorting signal induces translocation arrest and the lateral release of preproteins (lateral sorting) into the inner mitochondrial membrane by a stop- transfer mechanism (Van Der Laan et al. 2006; Bohnert et al. 2010). Lateral membrane integration of proteins depends on $\Delta \psi$ as the sole energy source. The inner membrane protease (IMP) cleaves off the hydrophobic sorting signal of some of these proteins, which subsequently remain soluble in the intermembrane space (Koppen \& Langer 2007; Mossmann et al. 2012; Teixeira \& Glaser 2013). 
Another type of inner membrane proteins is initially imported to the matrix in a PAMdependent manner and subsequently membrane-inserted with the help of the evolutionary conserved export translocase Oxa1 (Hell et al. 1998; Jia et al. 2007; Bohnert et al. 2010). This mechanism is known as "conservative sorting" (Neupert \& Herrmann 2007). Oxa1 closely cooperates with the TIM23-PAM machinery for the biogenesis of nuclear-encoded multi-spanning inner membrane proteins (Reif et al. 2005; Bohnert et al. 2010). The term "conservative sorting" was initially used for the sorting pathway of the Rieske iron-sulfur protein (Rip1). This protein consists of a large C-terminal IMS domain that contains the iron sulfur cluster. In vitro import experiments indicate that the entire precursor is initially imported into the matrix (Nett \& Trumpower 1996) where the iron-sulfur cluster is incorporated into the protein. The catalytic domain is subsequently exported into the IMS and only then assembled into the $b c_{1}$ complex (Golik et al. 2003). 


\subsubsection{Mitochondrial protein export}

Mitochondria-encoded membrane proteins that are translated on membrane bound mitochondrial ribosomes have to be integrated into the inner membrane in a co-translational manner. This process has been termed export and is facilitated by the mitochondrial export machinery (OXA - for oxidase assembly). The OXA complex consists of Oxa1 as core subunit (Nargang et al. 2002; Kohler et al. 2009). Oxa1 belongs to a conserved protein family known as YidC/Alb3/Oxa1 family, the bacterial, mitochondrial and plastid members of which assist the membrane insertion of proteins (Bonnefoy et al. 2009; Wang \& Dalbey 2011). Oxa1 is an integral inner membrane protein that contains five trans-membrane domains and presents $\mathrm{N}$ out-C in orientation (Luirink et al. 2001; Stoldt et al. 2012). By cooperation with the membrane protein Mba1, which acts as a ribosome receptor, 0xa1 orients the ribosome exit site towards the inner membrane insertion machinery (Ott et al. 2006; Hell et al. 2001). However, so far uncharacterized additional membrane anchors apparently exist, as both ribosomal subunits remain membrane-bound even in the absence of Oxa1 and Mba1.

Oxa1 is particularly involved in Cox 2 biogenesis: Cox 2 is synthesized as a precursor protein (pCox2) with a cleavable amino terminal extension. Prior to the cleavage, pCox2 interacts with the 0xa1 machinery which facilitates membrane insertion of the first pCox2 transmembrane domain and concomitant export of its $\mathrm{N}$-terminal domain across the inner membrane (reviewed in Soto et al. 2012).

However, Oxa1 is required not only for the membrane insertion of mitochondriaencoded proteins but is also a crucial factor for the export of nuclear-encoded subunits of respiratory chain complexes: 0xa1 mediates the conservative sorting pathway of presequence-containing inner membrane proteins that are initially transported into the matrix before they are inserted into the inner membrane (described in section 1.1.5.1). 


\subsubsection{Mitochondria associated diseases}

Mitochondrial diseases are a diverse group of disorders caused by dysfunctional mitochondria. These diseases may be the result of spontaneous or inherited mutations in the mitochondrial genome or in nuclear genes that code for mitochondrial components. In about $15 \%$ of the cases, mitochondrial function is affected by defects in the mitochondrial DNA (Dimauro \& Davidzon 2005). Diseases related to $m t$ DNA mutations are transmitted by non-mendelian, maternal inheritance. In addition, mitochondrial dysfunction may also be caused by secondary effects of drugs, infections, or other environmental causes. Mitochondrial disorders in humans are not rare: when studies in children and adults are combined and both nuclear DNA and mitochondrial DNA mutations are considered, the minimum prevalence is at least 1 in 5000 (Schaefer et al. 2004). Deficiencies in mitochondrial function often affect multiple tissues, leading to multi-systemic diseases that present with pleiotropic phenotypic features (Scharfe et al. 2009). The effects of mitochondrial disease can be quite varied since the distribution of the defective mitochondrial DNA varies from organ to organ within the body and each mutation is modulated by other genome variants. Defects in nuclear-encoded mitochondrial genes are associated with hundreds of clinical disease phenotypes including anemia, dementia, hypertension, lymphoma, retinopathy, seizures, and neurodevelopmental disorders. However, since heart, skeletal muscle, and brain are among the most energy-dependent tissues of the body, many mitochondrial disorders present themselves as encephalo-cardiomyopathies. Encephalomyopathies have traditionally been described as defects of the respiratory chain, however other important mitochondrial functions including protein import, organellar dynamics and programmed cell death can also be affected (Dimauro \& Schon 2008).

Mitochondrial encephalomyopathy, lactic acidosis, and stroke-like episodes (MELAS) is a family of mitochondrial disorders that are caused exclusively by defects in the mitochondrial genome. The MELAS family includes also the MERRF syndrome (Myoclonic Epilepsy with Ragged Red Fibers) and Leber's hereditary optic neuropathy (LHON). All pathogenic LHON point mutations affect genes in the mitochondrial DNA, encoding for subunits of complex I of the respiratory chain (Wallace et al. 1988; Dimauro \& Schon 2008). The MERRF syndrome is mainly caused by a point mutation in the mitochondrial gene for tRNA-Lys thereby disrupting the synthesis of proteins essential for oxidative phosphorylation (Dimauro et al. 2002). 
Leigh syndrome, an infantile subacute necrotizing encephalomyelopathy, caused by disorders of the oxidative phosphorylation system is implicated with mutations in either the mitochondrial or nuclear DNA. Over 30 genes have been linked to Leigh syndrome, including SURF1 and other assembly factors of cytochrome $c$ oxidase (Tiranti et al. 1998; Zhu et al. 1998; Y. Zhang et al. 2007; Finsterer 2008).

Leigh syndrome (LS) is a fatal neurodegenerative condition, pathologically characterized by subacute symmetrical necrotic lesions in the subcortical regions of the central nervous system, first described in 1951 (Leigh 1951). LS is the most common mitochondrial disorder of infancy with a prevalence of 1:40000 (Rahman et al. 1996). The syndrome results from impaired mitochondrial energy metabolism, in detail by respiratory chain defects or in rare cases defects of the pyruvate dehydrogenase complex. LS can be caused by maternally inherited mutations in the mtDNA, but inheritance can also be autosomal-recessive or X-linked. A number of different mutations in nuclear or mitochondrial genes are found in patients (Finsterer 2008): mutations affecting subunits of complex I, mutations affecting complex II, complex IV or the F1Fo-ATP-synthase. Over 30 genes have been linked to Leigh syndrome, including SURF1 and other assembly factors of cytochrome $c$ oxidase (Tiranti et al. 1998; Zhu et al. 1998; Y. Zhang et al. 2007; Finsterer 2008). Cytochrome $c$ oxidase deficiency is one of the most common causes of LS (Péquignot et al. 2001), but interestingly all the mutations described to date have been in genes coding for proteins required for cytochrome $c$ oxidase assembly and not for structural subunits. Leigh Syndrome with isolated cytochrome $c$ oxidase deficiency is most commonly caused by SURF1 mutations (Péquignot et al. 2001; Shoubridge 2001). These patients typically retain 10-20\% of cytochrome $c$ oxidase activity (Shoubridge 2001). In this study, we analysed a mutation affecting a conserved amino acid in SURF1 in its yeast homolog Shy1. The investigated

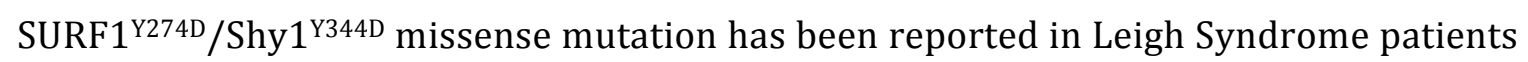
and thus is known to affect cytochrome $c$ oxidase biogenesis (Teraoka et al. 1999). 
Not only defects in mitochondrial metabolism but also disturbed mitochondrial biogenesis can lead to mitochondrial diseases. In Barth syndrome (BTHS), an X-linked genetic disorder, mutations in the tafazzin gene (TAZ) cause abnormalities in cardiolipin molecules (Barth et al. 2004). Cardiolipin, a phospholipid found in mitochondrial membranes, is closely related with membrane dynamics and energy metabolism, by providing stability for electron transport chain complexes (Vreken et al. 2000; Kulik et al. 2008).

Recently, secondary $m t$ DNA alterations are also speculated to be involved in aging and neurodegenerative disorders as Alzheimers disease, Parkinsons disease, Huntingtons disease or amytrophic lateral sclerosis. Neurons are highly dependent on oxidative energy metabolism. Therefore a common mechanism of neurodegeneration, based on an underlying dysfunction in mitochondrial energy metabolism, is under discussion (Schon \& Manfredi 2003; Dimauro \& Schon 2008). 


\subsection{Cytochrome $c$ oxidase}

\subsubsection{Structure and function}

Cytochrome $c$ oxidase (COX), the terminal enzyme of the respiratory chain, is a copperheme $a$ terminal oxidase, composed of multiple subunits (11 in S. cerevisiae, 13 in human) of dual genetic origin. The crystal structure of the dimeric bovine cytochrome $c$ oxidase revealed the spatial arrangement of the subunits and the positioning of the cofactors (Tsukihara et al. 1995; Tsukihara et al. 1996).

The three core subunits Cox1, Cox 2 and Cox3 are deeply buried in the complex and form the catalytic core (FIG 1.3). They are encoded by the mitochondrial genome and are highly conserved among respiring organisms (Castresana et al. 1994).

A

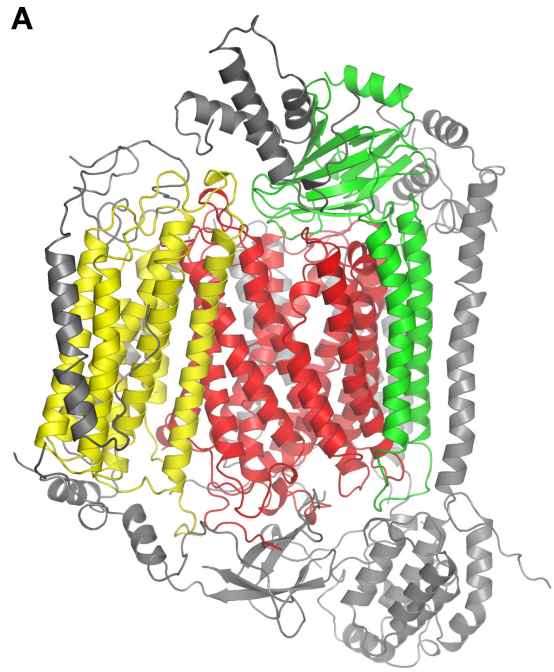

B

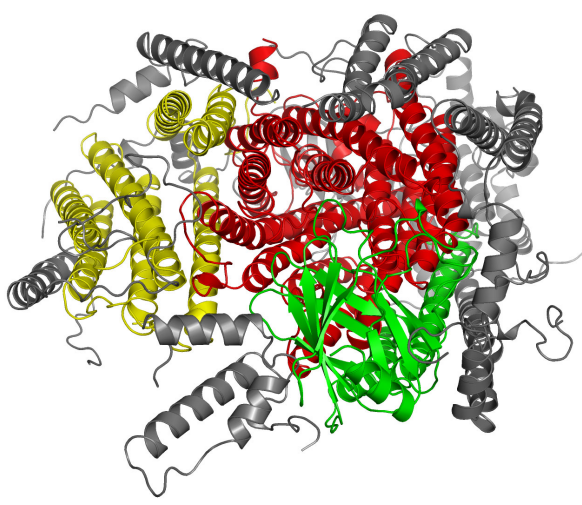

FIG 1.3 Monomeric bovine Cytochrome $c$ oxidase crystal structure

Shown are cartoons of mitochondria-encoded bovine subunits Cox1 (red), Cox2 (green) and Cox3 (yellow). Nuclear-encoded subunits in grey (Tsukihara et al. 1996). (A) Side view, matrix at the bottom and IMS at the top. (B) top view from the IMS side. PBD entry : 10CC, analysed with PyMOL software.

The structure of the metal sites of bovine cytochrome $c$ oxidase (Tsukihara et al. 1995) furthermore revealed that the redox cofactors heme and copper are inserted into the core proteins Cox1 and Cox2 (FIG 1.4). Cytochrome $c$ oxidase transfers electrons from cytochrome $c$ to molecular oxygen via the redox active metal cofactors in its catalytic core. Therefore, the cofactors are essential for the catalytic activity of the enzyme. 
Cox2 forms the binding pocket for reduced cytochrome c from which it acquires electrons that are passed to the binuclear $\mathrm{Cu}_{\mathrm{A}}$ site in $\mathrm{Cox} 2$, subsequently to the low spin heme $a$ in subunit 1 and finally to the active site where a high spin heme $a_{3}$ (in Cox1) and the $\mathrm{Cu}_{\mathrm{B}}$ site form a binuclear center for $\mathrm{O}_{2}$ binding (reviewed by Soto et al. 2012). In the final step, the electrons are transferred to molecular oxygen to form $\mathrm{H}_{2} \mathrm{O}$. This electron transport reaction is coupled to proton pumping from the matrix to the IMS (Yoshikawa et al. 2011). The precise function of Cox3 is not known, but it appears to form an aqueous channel, which may allow protons to move through the membrane (Brunori et al. 1987).

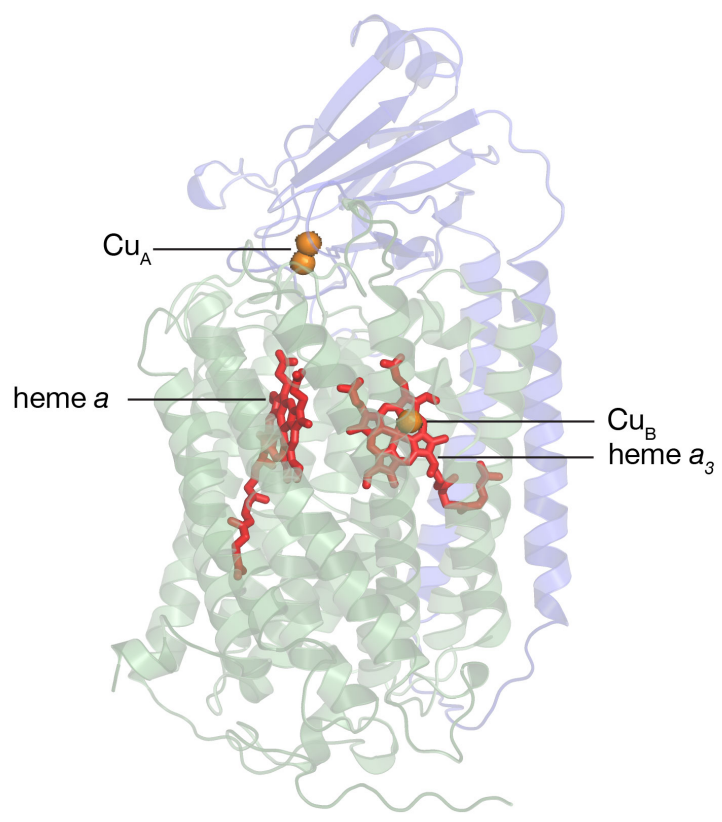

FIG 1.4 Cytochrome $c$ oxidase catalytic core

Arrangement of the hemes and copper centers in (bovine) cytochrome c oxidase catalytic core. Shown is a cartoon of mitochondria-encoded bovine subunits Cox1 (transparent green) and Cox2 (transparent purple), heme moieties (red stick models) and copper ions (orange spheres). The binuclear CuA center is located in Cox2 subunit and is the entrance site for electrons from reduced cytochrome $c$. Electrons are subsequently passed to the low-spin heme $a$ and then to the heterobimetallic heme $a_{3}: \mathrm{Cu}_{\mathrm{B}}$ center in Cox 1 where $\mathrm{O}_{2}$ reduction occurs. Side view, matrix at the bottom and IMS at the top. PBD entry : 10CC, analysed with PyMOL software. 
The catalytic active core subunits of cytochrome $c$ oxidase are surrounded by a set of nuclear-encoded proteins (Cox4, Cox5a/b, Cox6, Cox7, Cox8, Cox9, Cox12, Cox13 in yeast). The surrounding structural subunits fulfill functions in assembly and stability of the complex as well as in modulation of the catalytic activity. Most importantly, they are speculated to protect the core from oxidative damage. The importance of these subunits is demonstrated by the complete loss of COX activity of yeast strains deleted in the nuclear genes encoding for subunits Cox4, the two isoforms of Cox5 (Cox5a and Cox5b), Cox6, Cox7, and Cox9, suggesting an essential role for these subunits in assembly and stability of the enzyme (reviewed by Fontanesi et al. 2006).

Assembly of COX is thought to be a linear process where the different subunits are added in an ordered manner. Many (non-structural) factors that assist the assembly have been identified in yeast and distinct assembly intermediates can be resolved by BN-PAGE. Identification and characterization of mammalian counterparts of assembly factors, originally identified in yeast, are under ongoing research (Szklarczyk, Wanschers, Cuypers, et al. 2012a; Mick et al. 2012; Szklarczyk, Wanschers, Nijtmans, et al. 2012b; Clemente et al. 2013). The vast majority of mitochondrial disorders, presented with COX deficiency, have been assigned to mutations in these ancillary factors, among them mutations in SURF1 (required for formation of early intermediates) (Zhu et al. 1998), SCO1 and SCO2 (required for copper insertion) (Papadopoulou et al. 1999; Valnot, Osmond, et al. 2000b), in COX10 and COX15 (required for heme cofactor biogenesis) (Valnot, Kleist-Retzow, et al. 2000a; Antonicka et al. 2003) or in TACO1 (required for expression of subunits) (Weraarpachai et al. 2009). 


\subsubsection{Non-protein cofactors}

The incorporation of prosthetic groups into cytochrome $c$ oxidase subunits is essential for the catalytic activity of cytochrome $c$ oxidase. In addition, it is necessary for the maturation and correct folding of the Cox1 polypeptide (Carr \& Winge 2003). Despite the importance of the non-protein cofactors of cytochrome $c$ oxidase, little is known about when and how the metal groups are incorporated into the subunits during assembly of cytochrome $c$ oxidase.

\subsubsection{Heme $a$ biogenesis and insertion}

Heme $a$ is a prosthetic group present in cytochrome $c$ oxidase. Cytochrome $c$ oxidase is the only enzyme within the cell that requires heme $a$ as a cofactor. Heme $a$ is formed from heme $b$ (protoheme) in a two-step process. In the first step, catalyzed by the heme $o$ synthase Cox10 (in yeast), heme $b$ is farnesylated. In a second step, a methyl group in heme $o$ is oxidized to form a formyl group that gives rise to heme $a$ (Caughey et al. 1975). This reaction is catalyzed by the heme $a$ synthase Cox15, ferredoxin (Yah1) and a ferredoxin reductase (Arh1) (FIG 1.4). Studies in Bacillus subtilis have provided strong evidence that the heme $a$ synthase oxidizes heme $o$ to heme $a$ via two successive monooxygenase reactions (Brown et al. 2002).
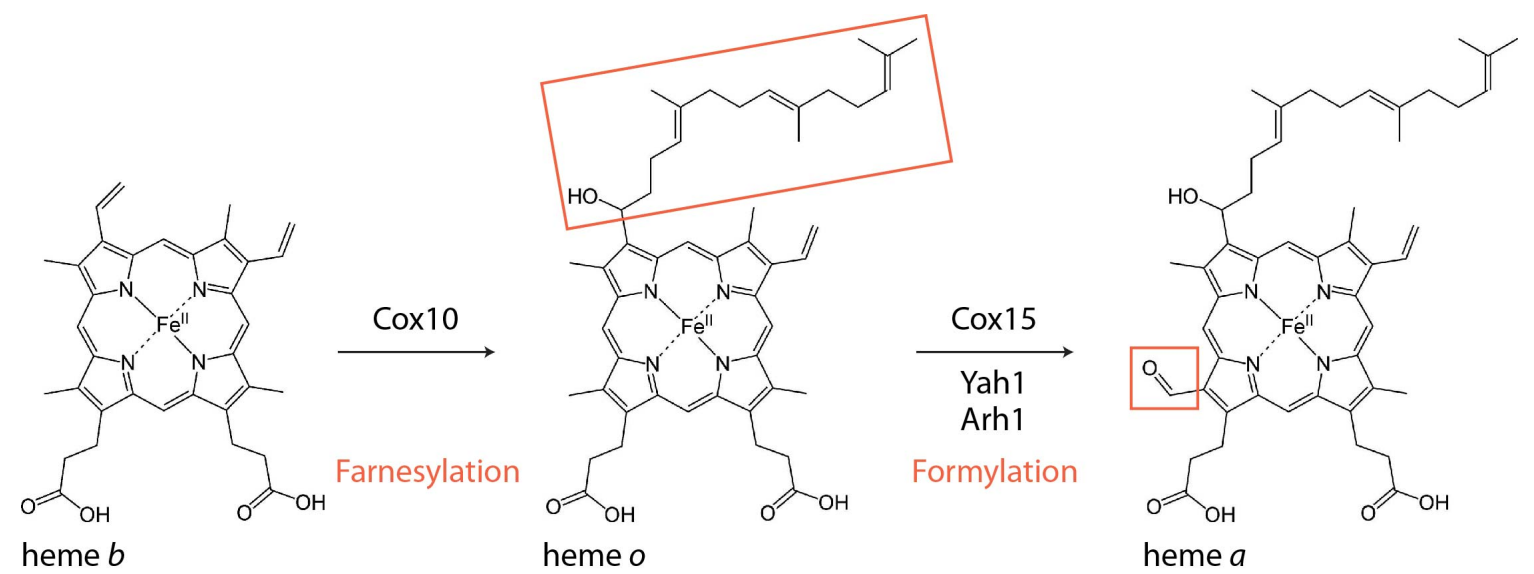

FIG 1.5 Biosynthesis of heme $o$ and heme $a$

Chemical modifications of heme $b$ to heme $a$ via heme $o$, catalyzed by the enzymes heme $o$ synthase (HOS, Cox10 in $S$. cerevisiae) and heme a synthase (HAS, Cox15 in S. cerevisiae ). Ferredoxin Yah1 and adrenodoxin Arh1 supply electrons for Cox15-mediated heme $o$ to heme $a$ conversion. 
Both Cox10 and Cox15 are hydrophobic multispanning membrane proteins, found in the inner mitochondrial membrane (Glerum \& Tzagoloff 1994; Barros et al. 2001). Because the heme $a$ cofactor is essential to cytochrome $c$ oxidase and Cox10 and Cox15 are both required for its synthesis, $\operatorname{cox} 10$ and $\operatorname{cox} 15$ deletions and mutations lead to a deficiency in respiratory growth. In both cases, cytochrome $c$ oxidase subunits are present but are not assembled into a functional enzyme (Nobrega et al. 1990; Glerum et al. 1997). The Cox15 mutant accumulates heme $o$ but lacks heme $a$ (Barros et al. 2001).

Homologs of the yeast Cox10 and Cox15 are found in various organisms from prokaryotes to humans. The human homolog of Cox10 is able to functionally complement the yeast cox10 deletion (Glerum \& Tzagoloff 1994). Mutations in the human COX10 and COX15 genes lead to cytochrome $c$ oxidase deficiency and have been associated with severe infantile cardiomyopathy, tubulopathy, leukodystrophy and Leigh syndrome (Valnot, Kleist-Retzow, et al. 2000a; Antonicka et al. 2003; Moraes et al. 2004; Oquendo 2004; Alfadhel et al. 2011).

Even though hemylation of Cox1 is essential, little is known about the insertion of the two heme moieties into Cox1. As heme is a very reactive moiety, it is highly likely that a heme-binding protein escorts it to its final destination. For the same rational, the biosynthesis of heme $a$ is regulated by downstream events in the COX assembly process in order to prevent accumulative of this reactive moiety (Barros \& Tzagoloff 2002). It is not clear if the insertion of the two hemes into Cox1 occurs co-translationally or later (post-translational) in the maturation of Cox1. However, it is believed that the insertion of heme $a_{3}$ has to occur before the addition of Cox2 as the farnesyl group of this heme is located at the interface between the two subunits (see also FIG 1.4) (Tsukihara et al. 1995).

Recent analyses support the idea that the bacterial homolog of Shy1 (Surf1 in Paracoccus denitrificans) plays a role in the insertion step for heme $a$, suggested by the heme-binding activity of a bacterial homologue. However, it remains unclear whether the bacterial homolog of Shy1 acts as a "heme-chaperone" or has a more direct role in heme insertion into subunit 1 of cytochrome $c$ oxidase (Bundschuh et al. 2009; Hannappel et al. 2011; Hannappel et al. 2012). 


\subsubsection{Copper}

In mitochondria, two enzymes, cytochrome $c$ oxidase and superoxide dismutase (Sod1) receive copper within the IMS (Cobine et al. 2006). This requires a specific copper transport pathway to this compartment of the organelle. Copper metallation of cytochrome $c$ oxidase involves the copper metallo-chaperone Cox17, a small hydrophilic protein that contains a copper binding motif (Glerum et al. 1996). The dual localization of Cox17 in the cytosol and within mitochondria is consistent with the proposed copper shuttle function of Cox17 to mitochondria (Beers et al. 1997). Within the IMS, Cox17 transfers copper ions to the two chaperones Sco1 (and its paralog Sco2) or Cox11. Sco1 and Cox11, two mitochondrial inner membrane proteins facilitate copper insertion into the $\mathrm{Cu}_{\mathrm{A}}$ site (in Cox2) and into the $\mathrm{Cu}_{\mathrm{B}}$ site (in Cox1), respectively (Horng et al. 2004). Recent investigations speculated that the $\mathrm{Cu}_{\mathrm{B}}$ site formation does occur simultaneously to the incorporation of the heme $a_{3}$ moiety into the heterobimetallic center. (Khalimonchuk et al. 2010). Several studies in S. cerevisiae suggest that Cox19, Cox23, Pet191, Cmc1 and Cmc2 are also part of a copper transfer pathway towards cytochrome $c$ oxidase (Horn \& Barrientos 2008).

The mitochondrial copper metabolism involves conserved elements. Homologues of the yeast genes COX11, COX17, SC01, COX19, COX23, PET191, CMC1 and CMC2 have been identified in higher organisms. Mutations in SCO1 for example are associated with mitochondrial disorders (Valnot, Osmond, et al. 2000b). 


\subsection{Early steps in cytochrome $c$ oxidase assembly}

Cytochrome $c$ oxidase assembly is a highly regulated process. Not only the subunits of dual genetic origin have to be assembled, also the non-protein cofactors have to be inserted into the central subunits. In yeast, over 20 assembly factors are known to assist in this process. As assembly intermediates are potential sources of reactive oxygen species (ROS), an intelligent feedback regulation mechanism evolved: assembly intermediates play a central role in feedback regulation of mitochondrial Cox1 protein synthesis in response to its assembly state (Barrientos et al. 2004; Mick et al. 2007; Pierrel et al. 2007; Fontanesi 2013). Therefore, accumulation of non-assembled Cox1 is prevented. This kind of translational regulation was termed control by epistasis of synthesis (Choquet et al. 2001). Most unassembled Cox1 (also Cox 2 and Cox3) are degraded by the ATP dependent AAA proteases of the inner mitochondrial membrane (Langer et al. 2001).

The starting point of the assembly of cytochrome $c$ oxidase is the mitochondria-encoded subunit 1 (Cox1). For the translation of COX1 mRNA on membrane bound mitochondrial ribosomes, two translational activators, Pet309 and Mss51, are required. After cotranslational membrane insertion, facilitated by 0xa1, Cox1 associates with the early assembly factors Coa3 and Coa14. This complex forms the platform for the association of further ancillary factors, such as Coa1 and Shy1 (FIG 1.6; 1-5). The early cytochrome $c$ oxidase assembly intermediates were termed COA complexes.

In parallel or in following, poorly understood steps, metal cofactors are incorporated into the catalytic core before the first nuclear-encoded structural subunits (Cox5 and Cox6) are added to the maturing enzyme complex .

These early steps in the biogenesis of cytochrome $c$ oxidase are interconnected with regulation of translation: binding of newly synthesized Cox 1 by Coa3 and Cox14 stabilizes Cox1 and prevents degradation by inner membrane proteases. In addition, by sequestration of Mss51 and Coa1 in this complex, Mss51 is inactivated and translation of Cox1 is shut down. Upon maturation of the assembly intermediates, Mss51 dissociates and is able to stimulate further rounds of COX1 translation (FIG 1.6). 


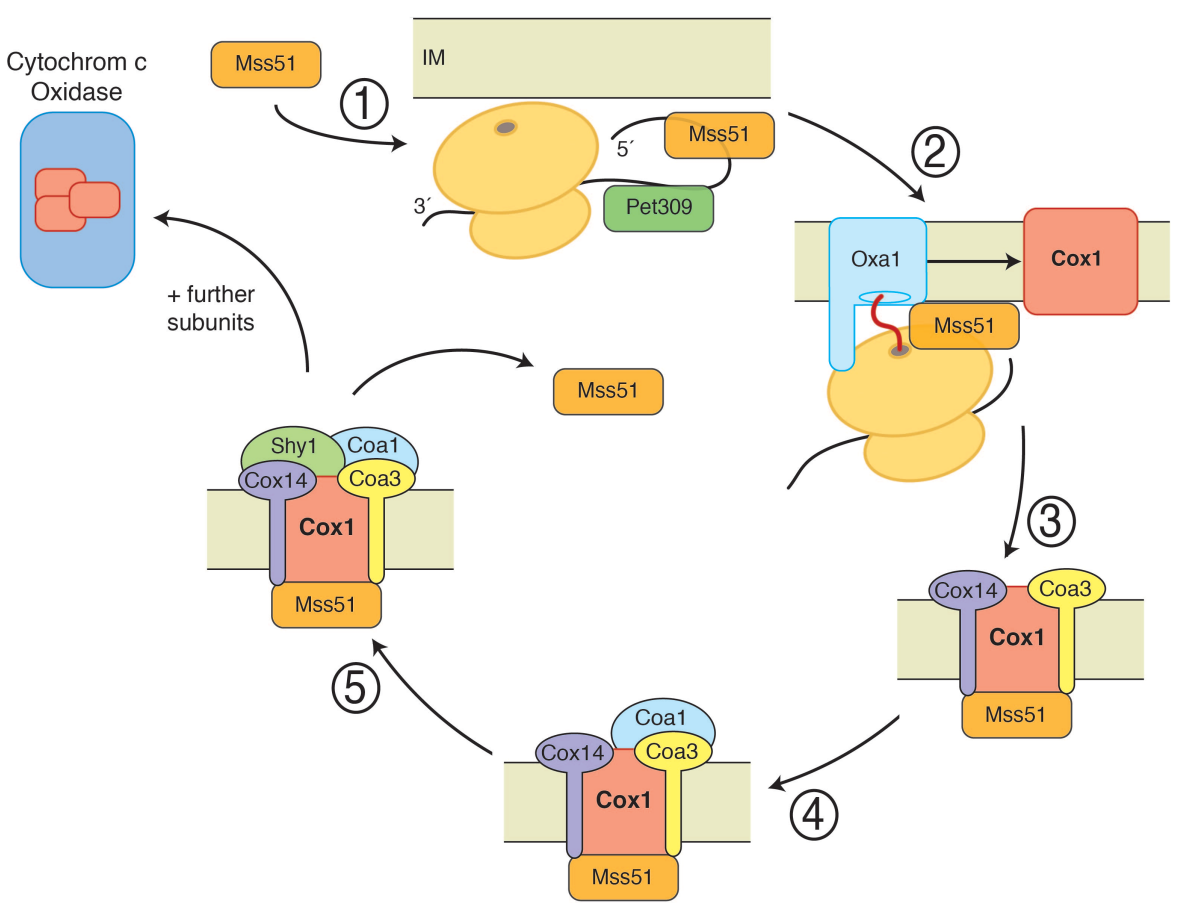

FIG 1.6 Early steps of cytochrome $c$ oxidase assembly and feedback regulation of Cox 1 translation (1) Mitochondrial splicing suppressor protein 51 (Mss51) activates COX1 translation, together with Pet309. (2) Cox1 is co-translationally inserted into the inner membrane (IM) by 0xa1. (3) Newly synthesized Cox1 is bound by Mss51. This interaction is promoted by early assembly factors (Coa3 and Cox14). (4) Assembly factor Coa1 is recruited to the complex. Mss51 is converted into an inactive form, preventing it to act as translational activator. (5) Coa1 association triggers binding of Shy1, which might be involved in insertion of heme cofactors into Cox1. Addition of further subunits (as nuclear-encoded Cox5 and Cox6) leads to the release of Mss51. Mss51 is again available to initiate further rounds of COX1 translation. The assembly intermediate is further matured to form the active cytochrome $c$ oxidase, that consists of 11 structural subunits in yeast.

\subsubsection{Membrane insertion of Cox1 by 0xa1}

Cox1 is a highly hydrophobic protein and spans the inner mitochondrial membrane with 12 trans-membrane domains. As Cox1 is being synthesized, it is co-translationally inserted into the inner mitochondrial membrane with the aid of the 0xa1 machinery (Hell et al. 2001; Bonnefoy et al. 2009). The carboxy-terminal domain of Oxa1 binds to mitochondrial ribosomes (Jia et al. 2003) near their exit-tunnel (Jia et al. 2009; Bonnefoy et al. 2009), which is consistent with a co-translational mechanism for the insertion of proteins, synthesized at mitochondrial ribosomes, into the inner membrane. 


\subsubsection{Regulation of Cox1 translation}

In S. cerevisiae, translation of mitochondria-encoded mRNAs, specifying for subunits of respiratory complexes, requires dedicated translational activator proteins, which recognize the $5^{\prime}$ untranslated regions (UTRs) of their target mRNAs. In the case of cytochrome $c$ oxidase subunit 1, Pet309 and the mitochondrial splicing suppressor protein 51 (Mss51) act as translational activators for COX1 mRNA (Perez-Martinez et al. 2003; Towpik 2005). Genetic studies show interaction of Mss51 with the 5'-UTR of COX1 mRNA (Perez-Martinez et al. 2003; Zambrano et al. 2007). Moreover, Mss51 interacts with newly synthesized but unassembled Cox1 (Perez-Martinez et al. 2009). Thus, Mss51 interacts both with COX1 mRNA and with Cox1 protein that has not been assembled into the mature cytochrome $c$ oxidase complex. These dual activities allow Mss51 to couple the synthesis of Cox 1 to the assembly of cytochrome $c$ oxidase via a regulatory feedback mechanism (Perez-Martinez et al. 2003; Barrientos et al. 2004; Perez-Martinez et al. 2009).

\subsubsection{Coa3 and Cox14 stabilize newly synthesized Cox1 and regulate COX1 translation}

Cox14 is a small protein of the inner mitochondrial membrane, involved in translational regulation of COX1 and the assembly of cytochrome $c$ oxidase. The majority of Cox14 associates with cytochrome $c$ oxidase assembly intermediates, however a fraction is also found in complex III/ IV supercomplexes (Glerum et al. 1995; Barrientos et al. 2004; Mick et al. 2007). Similarly, Coa3 (cytochrome $c$ oxidase assembly factor 3, termed Cox25 in another study) (Fontanesi et al. 2011) is a small mitochondrial inner membrane protein that regulates COX1 translation but also participates in Cox1 stabilization and cytochrome $c$ oxidase assembly (Mick et al. 2010; Fontanesi et al. 2011). Coa3 was identified in Shy $1^{\text {ProtA }}$ isolation of cytochrome c oxidase intermediates (Mick et al. 2010). The same study also found that Coa3 and Cox14, both containing a single transmembrane span, adopt the same topology in the inner mitochondria membrane and expose their C-termini into the IMS. The topology of Cox14 is in contrast to a previous study that found Cox14 to behave as a peripheral membrane protein (Barrientos et al. 2004). 
Newly synthesized Cox1 rapidly associates with Cox14 and Coa3, two small inner membrane proteins with domains that are exposed to the mitochondrial matrix and the intermembrane space (Glerum et al. 1995; Barrientos et al. 2004; Mick et al. 2010). These proteins then form a complex that is thought to keep Mss51 in a sequestered, but still active state (Mick et al. 2007). Cox14 and Coa3 are essential for Mss51 recruitment to Cox1, a first step and prerequisite for its subsequent inactivation by Coa1. Therefore, a lack of Coa3 or Cox14 leads to uncontrolled expression of COX1 due to a loss of the Mss51 negative feedback regulation (Mick et al. 2010). In consequence, unassembled Cox1 is rapidly turned over in these mutants, resulting in a specific lack of cytochrome $c$ oxidase and absence of respiratory growth (Mick et al. 2010).

Cox14 and Coa3 both negatively regulate Cox1 synthesis, however the proteins fulfill distinct functions as overexpression of either Cox14 in coa3 $\Delta$ cells or of Coa3 in cox $14 \Delta$ cells has no effect on the growth phenotype (Mick et al. 2010).

\subsubsection{Coa1 is required for progression of COX assembly}

Cytochrome $c$ oxidase assembly factor 1 (Coa1) is a mitochondrial inner membrane protein required for assembly of the cytochrome $c$ oxidase complex (Sickmann et al. 2003; Reinders et al. 2006; Mick et al. 2007; Pierrel et al. 2007). The amino-acid sequence predicts a protein of 197 amino acids with a molecular mass of about $22 \mathrm{kDa}$ with one transmembrane span (Mick et al. 2007). The N-terminus of Coa1 is rich in positively charged amino acids, characteristic of a mitochondrial presequence. A cleavage site for the mitochondrial processing peptidase is predicted adjacent to the presequence (Gakh et al. 2002). Coa1 is present in early assembly intermediates of cytochrome $c$ oxidase (COA complexes). The association of Coa1 with the Cox1-Mss51Cox14-Coa3 complex in wild-type cells is required for the conversion of Mss51 to a sequestered inactive state, which prevents Mss51 from activating translation (see FIG 1.6; 4). Moreover, Coa1 association promotes the binding of Shy1 to Cox1, which might positively regulate the insertion of the heme cofactor into Cox1 (Mick et al. 2011). Cells lacking Coa1 have a specific defect in cytochrome $c$ oxidase assembly (Pierrel et al. 2007). This respiratory defect in coa1 1 cells is suppressed by high-copy MSS51 and COX10 (Pierrel et al. 2007). Translation of the mitochondria-encoded subunits of cytochrome $c$ oxidase occurs normally in coa1s cells, but these subunits fail to accumulate. 


\subsubsection{Additional function of assembly factor Shy1 in heme transfer to Cox1?}

Shy1, the yeast homolog of mammalian SURF1 is a mitochondrial inner membrane protein, with a large domain, exposed to the intermembrane space. The protein is essential for respiratory growth (Mashkevich et al. 1997) since it is required for regulation of COX1 expression and cytochrome $c$ oxidase assembly (Barrientos et al. 2002; Mick et al. 2007). Mutations in human SURF1 are reported to cause Leigh syndrome, a severe neurodegenerative disorder (Zhu et al. 1998; Tiranti et al. 1998). Similar to patients affected in SURF1 function, shy1 mutant mitochondria are characterized by reduced amounts of Cox1 and reduced levels of active cytochrome $c$ oxidase (Nijtmans et al, 2001; Barrientos et al, 2002; Williams et al, 2004). Although Shy1 does not directly regulate COX1 translation, it is implicated in post-translational steps of Cox1 biogenesis and early steps of cytochrome $c$ oxidase assembly through association with several membrane protein complexes (Mashkevich et al, 1997; Barrientos et al, 2002; Perez-Martinez et al, 2003; Smith et al, 2005; Zambrano et al, 2007). Shy 1 associates with Cox 1 assembly intermediates, containing the translational regulator Mss51 and early assembly factors, such as Coa3, Cox14 and Coa1. In addition, it can be identified as a component of COA complexes that already have the nuclearencoded subunit Cox5a incorporated (Mick et al. 2007). Recent analyses support the idea that Shy1 (or its homologues in other organisms) plays an important part in the insertion step of heme $a$ into Cox1, suggested by the heme binding activity of a bacterial homologue Surf1 (Bundschuh et al. 2009; Khalimonchuk et al. 2010). The recently identified assembly factors Coa2 and Coa4 appear to cooperate with Shy1 for heme insertion into Cox1 (Pierrel et al. 2008; Bestwick, Jeong, et al. 2010a). 


\subsubsection{Early COX assembly steps in higher eukaryotes}

Mutations in known human COX assembly factors (SURF1, SC01, COX10, COX15) lead to severe mitochondrial disorders (described in section 1.1.6). Analyses of patients suffering from mitochondrial encephalo-myopathies, such as Leigh Syndrome, contributed significantly to our current knowledge on human respiratory chain biogenesis.

However, many of the early assembly factors studied in yeast lack robust mammalian homologs and their identification is a topic of recent research: An in silico analysis that aimed to identify proteins with sequence similarity to these yeast factors revealed potential candidates, among them predicted homologs of multiple genes that control the COX1 translation process in fungi (C7orf44, CCDC56, C12orf62) (Szklarczyk, Wanschers, Cuypers, et al. 2012a). The same study confirmed that C12orf62 is a protein involved in the assembly of cytochrome $c$ oxidase in vivo. Moreover in a patient, a mutation in C12orf62 was reported to cause Fatal Neonatal Lactic Acidosis. Patient fibroblasts show impaired cytochrome $c$ oxidase assembly. C12orf62 is speculated to be required for coordination of the early steps of COX assembly with the translation of COX1 (Weraarpachai et al. 2012).

The newly identified human assembly factor MITRAC12 (previously termed CCDC56) was found to interact with newly synthesized COX1 in assembly intermediates. Surprisingly, TIM21, a subunit of the presequence translocase of the inner mitochondrial membrane is also present in these assembly intermediates, termed MITRAC complexes. MITRAC12 is required for efficient COX1 synthesis and assembles with other assembly factors such as SURF1, COX15, C12orf62 and C7orf44/MITRAC15 (Mick et al. 2012). Mitochondrial COX1 translation is regulated by the MITRAC complex and links the TIM23-dependent protein import to the assembly of respiratory-chain complexes.

A homolog or ortholog of Mss51 has not been identified so far. However, conservation of the translational regulation mechanism in the mammalian system is difficult to imagine due to the lack of extended $5^{\prime}$-UTR regions for binding of any regulating proteins. Therefore, the general existence of mRNA-specific translational factors in mammalian mitochondria is a subject of speculation. If these factors exist in the mammalian system, they are likely to interact with the coding sequence. 


\subsection{Research objectives}

Cytochrome $c$ oxidase assembly from multiple subunits of dual genetic origin is a highly complicated and regulated process. In yeast, over 20 assembly factors are involved in translation of mitochondria-encoded subunits as well as in their assembly and maturation. Significant amount of data concerning early steps of COX assembly has been accumulated; however the understanding, especially of the mechanism of cofactor insertion, is far from being complete.

The aim of this study was to analyse different stages in the early steps of COX assembly in order to extend our understanding of these processes in the yeast Saccharomyces cerevisiae. The investigation has a great biomedical relevance since lesions affecting conserved human COX assembly factors (e.g. SURF1, SC01, COX10, COX15) result in severe mitochondrial disorders such as Leigh syndrome.

This study dissected the importance of close proximity of the two 0xa1 domains in respect to COX assembly by using linker insertion mutants. Therefore, assembly of radiolabeled subunits of cytochrome $c$ oxidase into complexes was analysed.

In addition, the translational regulation process of $C O X 1$ was investigated by isolation of Mss51-containing complexes. In order to decrease sample variation, the purification was performed after stable isotope labeling with amino acids in cell culture (SILAC).

Furthermore, this study analysed a mutation affecting a conserved amino acid in SURF1, transferred to its yeast homolog Shy1. The investigated SURF1Y274D/Shy1 ${ }^{\text {Y344D }}$ missense mutation has been reported in Leigh Syndrome patients and thus is known to affect COX biogenesis. Using strains, expressing Shy $1^{\mathrm{YD}}$, the coupling of COX1 translation to COX assembly was addressed.

The last part of the study aimed to get a better understanding of the exact molecular role of Shy1 in assembly of cytochrome $c$ oxidase. It was previously speculated that heme $a$ gets inserted into Cox1 within the assembly intermediate complexes, most likely via Shy1 and its bacterial homolog Surf1. Therefore, Shy1 containing complexes were characterized in an unbiased manner. For this purpose, native isolation and separation of assembly intermediate complexes using BN-PAGE was combined with analysis of the components by mass spectrometry and Western blotting. The finding that Cox 15 , the heme $a$ insertase interacts with Shy1 and COA complexes lead to additional analyses of mutants, defective in heme biogenesis. 


\section{MATERIALS AND METHODS}

\subsection{Materials}

\subsubsection{Chemicals}

All standard chemicals were purchased from AppliChem (Darmstadt), Merck (Darmstadt), Roth (Karlsruhe), SERVA (Heidelberg) or Sigma-Aldrich (Taufkirchen) in analytical grade purity. Exceptions and special chemicals are listed in Table 7.

\subsubsection{Enzymes}

TABLE 1: Enzymes and suppliers

\begin{tabular}{l|l} 
Enzyme & Supplier \\
\hline \hline & \\
Creatine kinase & Roche \\
Proteinase K & Roche \\
AcTEV protease & Invitrogen \\
Zymolyase 20T & Seikagaku Biobusiness
\end{tabular}

\subsubsection{Kit systems}

TABLE 2: Kits used in this study

\begin{tabular}{l|l} 
Kit & Supplier \\
\hline \hline & \\
Flexi® Rabbit Reticulocyte Lysate System & Promega \\
KOD Hot Start DNA polymerase & Novagen \\
MEGAclear ${ }^{\mathrm{T} M}$ Kit & $\begin{array}{l}\text { Ambion (Life technologies) } \\
\text { Ambion (Life technologies) }\end{array}$ \\
QuickChange® Site-Directed Mutagenesis Kit & Stratagene/ Agilent \\
TNT® Quick coupled Transcription/Translation Systems & Promega \\
Wizard® Plus SV Minipreps DNA Purification System & Promega \\
Wizard® SV Gel and PCR Clean-Up System & Promega
\end{tabular}

Kit systems were used according to the manufacturers specifications. 


\subsubsection{Buffers and solutions}

TABLE 3: Composition of buffers and solutions

\begin{tabular}{|c|c|}
\hline Buffer/solution & Composition \\
\hline Acetate buffer & $0.5 \mathrm{M} \mathrm{NH}_{4} \mathrm{CH}_{3} \mathrm{COOH} / \mathrm{CH}_{3} \mathrm{COOH}, \mathrm{pH} 3.4$ \\
\hline Blotting buffer & $20 \mathrm{mM}$ Tris, $0.02 \%$ SDS, $150 \mathrm{mM}$ glycine, $20 \%$ ethanol \\
\hline BN acrylamide solution & $48 \%$ acrylamide, $1,5 \%$ bisacrylamide $(32: 1)$ \\
\hline BN Anode buffer & 50 mM Bis-Tris/HCl (pH 7.0) \\
\hline BN Cathode buffer & $\begin{array}{l}50 \mathrm{mM} \text { tricine, } 15 \mathrm{mM} \text { Bis-Tris, } 0.02 \% \text { Coomassie } \\
\text { Brilliant Blue G- } 250\end{array}$ \\
\hline BN gel buffer $(3 x)$ & $\begin{array}{l}200 \mathrm{mM} \text { 6-aminohaxanoic acid, } 150 \mathrm{mM} \text { Bis-Tris/HCl } \\
\text { (pH 7.0) }\end{array}$ \\
\hline BN sample buffer (10x) & $\begin{array}{l}\text { 5\% Coomassie brilliant blue G-250, } 500 \mathrm{mM} \\
\text { 6-aminohaxanoic acid, } 100 \mathrm{mM} \text { Bis-Tris/HCl (pH 7.0) }\end{array}$ \\
\hline Coomassie destainer & $30 \%$ ethanol, $10 \%$ acetic acid \\
\hline Coomassie stainer & $\begin{array}{l}40 \% \text { ethanol, } 10 \% \text { acetic acid, } 0.15 \% \text { Coomassie } \\
\text { Brilliant Blue R250 }\end{array}$ \\
\hline Digitonin buffer & $\begin{array}{l}20 \mathrm{mM} \text { Tris/HCl (pH 7.4), } 100 \mathrm{mM} \mathrm{NaCl}, 10 \% \text { glycerol, } \\
5 \mathrm{mM} \text { EDTA, } 2 \mathrm{mM} \text { PMSF, } 1 \% \text { digitonin }\end{array}$ \\
\hline Digitonin wash buffer & $\begin{array}{l}20 \mathrm{mM} \text { Tris/ } \mathrm{HCl}(\mathrm{pH} 7.4), 0.1 \mathrm{M} \mathrm{NaCl}, 10 \% \text { glycerol, } \\
5 \mathrm{mM} \text { EDTA, } 2 \mathrm{mM} \text { PMSF, } 0.3 \% \text { digitonin }\end{array}$ \\
\hline SEM & $250 \mathrm{mM}$ sucrose, $1 \mathrm{mM}$ EDTA, $10 \mathrm{mM}$ MOPS \\
\hline SDS loading buffer & $\begin{array}{l}10 \% \text { glycerol, } 2 \% \text { SDS, } 0.01 \% \text { Bromphenol blue, } \\
1 \% \beta \text {-mercaptoethanol, } 60 \text { mM Tris, pH } 6.8\end{array}$ \\
\hline SDS resolving gel buffer $(5 x)$ & 1.87 M Tris/HCl (pH 8.8) \\
\hline SDS running buffer & 25 mM Tris, 192 mM glycine, 0.1\% SDS \\
\hline SDS stacking gel buffer (10x) & $0.8 \mathrm{M}$ Tris/HCl (pH 6.8) \\
\hline TBS-T & 20 mM Tris/HCl (pH 7.5), 125 mM NaCl, 0.1\% Tween20 \\
\hline TE buffer & 10 mM Tris/HCl (pH 7.5), 1 mM EDTA \\
\hline TAE buffer & 40 mM Tris/acetate (pH 8.0), 2 mM EDTA \\
\hline
\end{tabular}




\subsubsection{Culture Media}

TABLE 4: Composition of culture media used in this study

\begin{tabular}{|c|c|}
\hline Media & Components \\
\hline LB & $1 \%$ bacto tryptone, $0.5 \%$ yeast extract, $1 \% \mathrm{NaCl}, \mathrm{pH} 7.0$ \\
\hline LB-Amp & $\begin{array}{l}1 \% \text { bacto tryptone, } 0.5 \% \text { yeast extract, } 1 \% \mathrm{NaCl}, 100 \mathrm{mg} / \mathrm{ml} \\
\text { ampicillin, pH } 7.0\end{array}$ \\
\hline SD-His & $0.67 \%$ yeast nitrogen base, $0.07 \% \mathrm{CSM}-\mathrm{His}, 2 \%$ glucose \\
\hline SD-Ura & $0.67 \%$ yeast nitrogen base, $0.07 \%$ CSM-Ura, $2 \%$ glucose \\
\hline SGal-Trp & $0.67 \%$ yeast nitrogen base, $0.07 \%$ CSM-Trp, $2 \%$ galactose \\
\hline SGal-Ura & $0.67 \%$ yeast nitrogen base, $0.07 \% \mathrm{CSM}-\mathrm{Ura}, 2 \%$ galactose \\
\hline SG-His & $0.67 \%$ yeast nitrogen base, $0.07 \%$ CSM-His, $3 \%$ glycerol \\
\hline SILAC & $\begin{array}{l}0.67 \% \text { yeast nitrogen base, } 20 \mathrm{mg} / \mathrm{l} \text { histidine, } 20 \mathrm{mg} / \mathrm{l} \text { tryptophane, } \\
20 \mathrm{mg} / \mathrm{l} \text { adenine, } 20 \mathrm{mg} / \mathrm{l} \text { methionine, } 20 \mathrm{mg} / \mathrm{l} \text { uracil, } 30 \mathrm{mg} / \mathrm{l} \\
\text { isoleucine, } 30 \mathrm{mg} / \mathrm{l} \text { tyrosine, } 50 \mathrm{mg} / \mathrm{l} \text { phenylalanine, } 100 \mathrm{mg} / \mathrm{ml} \\
\text { leucine, } 150 \mathrm{mg} / \mathrm{l} \text { valine, } 200 \mathrm{mg} / \mathrm{l} \text { threonine, } 200 \mathrm{mg} / \mathrm{l} \text { proline, } \\
20 \mathrm{mg} / \mathrm{l} \text { (heavy or light, respectively) lysine, } 20 \mathrm{mg} / \mathrm{l} \text { (heavy or light, } \\
\text { respectively) arginine }\end{array}$ \\
\hline 2x YPAD & $\begin{array}{l}2 \% \text { yeast extract, } 4 \% \text { peptone, } 4 \% \text { glucose, } 100 \mathrm{mg} / \mathrm{l} \text { adenine } \\
\text { hemisulfate, } \mathrm{pH} 6.0\end{array}$ \\
\hline YPD & $1 \%$ yeast extract, $2 \%$ peptone, $2 \%$ glucose \\
\hline YPGal & $1 \%$ yeast extract, $2 \%$ peptone, $2 \%$ galactose \\
\hline YPG & $1 \%$ yeast extract, $2 \%$ peptone, $3 \%$ glycerol, pH 5.0 \\
\hline
\end{tabular}

All media were either autoclaved or filter-sterilized. For solid media plates, 2.5\% agar was added before autoclaving.

\subsubsection{Cell lines}

All yeast (Saccharomyces cerevisiae) strains used in this study were derivatives of YPH499 (Sikorski \& Hieter 1989), with the exception of cox1- (derivative of 777-3A) (Netter et al. 1982), cox2- (derivative of AB1-4D) (Kruszewska et al. 1980) and

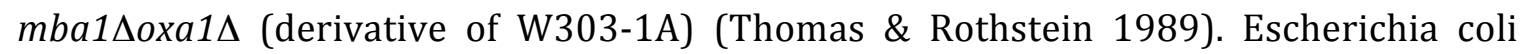
(E.coli) strain XL1-blue (Stratagene) was used for molecular cloning in this study. 
TABLE 5: Genotype and source of yeast strains used in this study

\begin{tabular}{|c|c|c|}
\hline Strains & Genotype & Reference or source \\
\hline YPH499 & MATa, ade2-101 his3- $\Delta 200$ leu2- $\Delta 1$ ura3-52 trp1- $\Delta 63$ lys2-801 & Sikorski \& Hieter 1989 \\
\hline Shy1 ProtA & $\begin{array}{l}\text { MATa, ade2-101 his3- } \Delta 200 \text { leu2- } \Delta 1 \text { ura3-52 trp1- } 663 \text { lys2-801; shy1::shy1-TEV- } \\
\text { ProtA-7HIS-HIS3MX6 }\end{array}$ & Mick et al. 2007 \\
\hline shy1s & MATa, ade2-101 his3- $\Delta 200$ leu2- $\Delta 1$ ura3-52 trp1- $\Delta 63$ lys2-801; shy1::HIS3MX6 & Mick et al. 2007 \\
\hline $\operatorname{cox} 1^{-}$ & MAT $\alpha$, ade 1 op $1 ; \operatorname{cox} 1-\mathrm{G} 421$ & Netter et al. 1982 \\
\hline $\cos 2^{-}$ & MAT $\alpha$, ade1 op1 met3; cox2-V25 & Kruszewska et al. 1980 \\
\hline Сoа3 $3^{\mathrm{HA}}$ & $\begin{array}{l}\text { MATa, ade2-101 his3- } \Delta 200 \text { leu2- } \Delta 1 \text { ura3-52 trp1- } \Delta 63 \text { lys2-801; coa3::coa3-3HA- } \\
\text { HIS3MX6 }\end{array}$ & Mick et al. 2010 \\
\hline $\operatorname{coa} 3 \Delta$ & MATa, ade2-101 his3- $\Delta 200$ leu2- $\Delta 1$ ura3-52 trp1- $\Delta 63$ lys2-801; coa3:: HIS3MX6 & Mick et al. 2010 \\
\hline $\operatorname{cox} 14 \Delta$ & $\begin{array}{l}\text { MATa, ade2-101 his3- } \Delta 200 \text { leu2- } \Delta 1 \text { ura3-52 trp1- } \Delta 63 \text { lys2-801; cox14:: } \\
\text { HIS3MX6 }\end{array}$ & Mick et al. 2010 \\
\hline Mss $51^{\mathrm{HA}}$ & 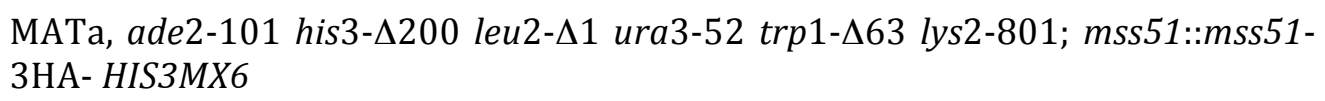 & Mick et al. 2010 \\
\hline shy $1 \Delta+$ pRS416 & $\begin{array}{l}\text { MATa, ade2-101 his3- } \Delta 200 \text { leu2- } \Delta 1 \text { ura3-52 trp1- } \Delta 63 \text { lys2-801; shy1::HIS3MX6; } \\
\text { [pRS416] }\end{array}$ & Reinhold et al. 2011 \\
\hline $\operatorname{shy} 1 \Delta+$ Shy1wT & $\begin{array}{l}\text { MATa, ade2-101 his3- } \Delta 200 \text { leu2- } \Delta 1 \text { ura3-52 trp1- } 663 \text { lys2-801; shy1::HIS3MX6; } \\
\text { [pRS416-SHY1] }\end{array}$ & Reinhold et al. 2011 \\
\hline $\operatorname{shy} 1 \Delta+$ Shy1YD & 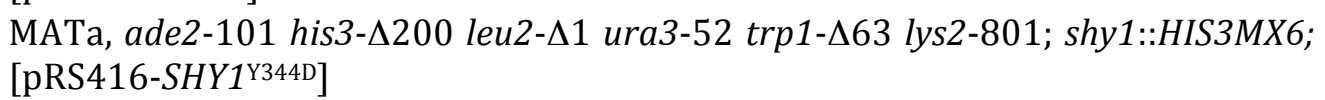 & Reinhold et al. 2011 \\
\hline$m b a 1 \Delta$ oxa1s + pRS424 & $\begin{array}{l}\text { MATa, ade2-1 his3-11,15 leu2-3, } 112 \text { ura3-1 trp1-1; mba1:: HIS3MX6; } \\
\text { oxa1::LEU2 [pRS424] }\end{array}$ & Keil et al. 2012 \\
\hline $\operatorname{mba} 1 \Delta$ oxa1s + 0xa1wT & $\begin{array}{l}\text { MATa, ade2-1 his3-11,15 leu2-3, } 112 \text { ura3-1 trp1-1; mba1:: HIS3MX6; } \\
\text { oxa1::LEU2 [pRS424-OXA1] }\end{array}$ & Keil et al. 2012 \\
\hline$m b a 1 \Delta$ oxa $1 \Delta+0 \times a 1100$ & $\begin{array}{l}\text { MATa, ade2-1 his3-11,15 leu2-3, } 112 \text { ura3-1 trp1-1; mba1:: HIS3MX6; } \\
\text { oxa1::LEU2 [pRS424-OXA1100] }\end{array}$ & Keil et al. 2012 \\
\hline$m b a 1 \Delta o x a 1 \Delta+0 \times a 1200$ & $\begin{array}{l}\text { MATa, ade2-1 his3-11,15 leu2-3, } 112 \text { ura3-1 trp1-1; mba1:: HIS3MX6; } \\
\text { oxa1::LEU2 [pRS424-OXA1200] }\end{array}$ & Keil et al. 2012 \\
\hline Mss51SF (BBY45) & 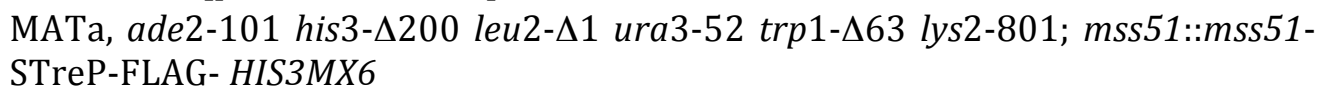 & This study \\
\hline
\end{tabular}




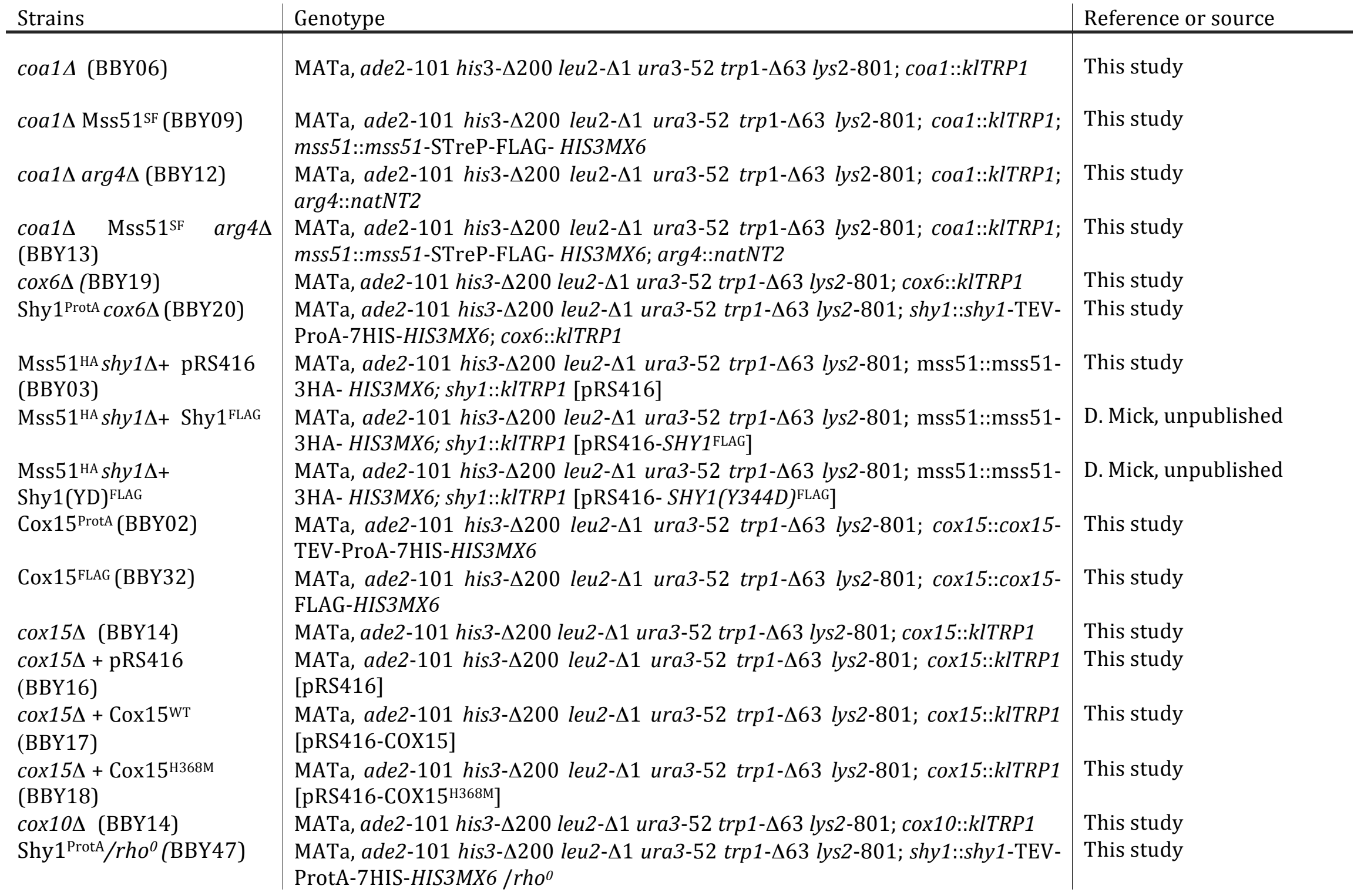




\subsubsection{Plasmids}

All plasmids used in this study are listed in table 6. Plasmids generated for this study were obtained by site directed mutagenesis of parental plasmids, as described in section 2.4.7. Plasmids were propagated in E.coli XL1-blue cells.

TABLE 6: Plasmids used in this study

\begin{tabular}{|c|c|c|c|}
\hline Plasmid & Backbone & Insert & Source \\
\hline pRS416 & - & - & Stratagene \\
\hline pDaM20 (Shy1 ${ }^{\text {WT }}$ ) & pRS416 & SHY1 b & Reinhold et al. 2011 \\
\hline pDaM23 (Shy1 $1^{\mathrm{Y} 34 \mathrm{D}}$ ) & pDaM20 & SHY1-(T1030G) & Reinhold et al. 2011 \\
\hline pDaM55 (Cox15wT) & pRS416 & $\operatorname{cox} 15^{a}$ & D. Mick, unpublished \\
\hline pBB06 (Cox15 $\left.5^{\mathrm{H} 368 \mathrm{M}}\right)$ & pDaM55 & COX15-(CAT1102ATG) & This study \\
\hline C21 (Cox $5 a)$ & pGEM4 & COX5A & AG Rehling (C21) \\
\hline
\end{tabular}

a: including $262 \mathrm{bp}$ upstream and $292 \mathrm{bp}$ downstream of the open reading frame b: including $372 \mathrm{bp}$ upstream and $363 \mathrm{bp}$ downstream of the open reading frame

\subsubsection{Antibodies}

Polyclonal primary antibodies were raised in rabbit either against a C-terminal peptide or against the whole protein. Secondary antibodies against rabbit immunoglobulin, coupled to horseradish-peroxidase were used for Western blot detection.

Monoclonal primary antibodies against FLAG and HA were purchased and detected with IgG goat anti mouse, coupled to HRP.

For detection of protein A, Peroxidase anti-peroxidase (PAP) soluble complex, produced in rabbit was purchased and signals were enhanced by secondary antibodies against rabbit immunoglobulin, coupled to horseradish-peroxidase. 


\subsubsection{Special chemicals}

TABLE 7: Special chemicals and their suppliers

\begin{tabular}{|c|c|}
\hline Chemical & Supplier \\
\hline Anti- HA, mouse monoclonal antibody & Roche \\
\hline ATP & Roche \\
\hline Coomassie brilliant blue G250/ R250 & Serva \\
\hline Creatine phosphate & Roche \\
\hline Desthiobiotin & IBA \\
\hline Digitonin & Calbiochem \\
\hline Dropout mixes (CSM-His/CSM-Trp/CSM-Ura) & MP Biomedicals \\
\hline ECL Western Blotting detection reagent & GE-Healthcare \\
\hline FLAG agarose, FLAG peptide & Sigma \\
\hline GeneRuler DNA Ladder Mix & Fermentas \\
\hline Goat anti rabbit HRP & Dianova \\
\hline Goat anti mouse HRP & Dianova \\
\hline Herring sperm DNA & Promega \\
\hline HMW calibration Kit & GE Healthcare \\
\hline IgG (human) & Sigma \\
\hline IgG standard (bovine) & Biorad \\
\hline L-Lysine:2HCl (U-13C6, 99\%; U-15N2, 99\%) & Cambridge Isotope Laboratories \\
\hline L-Arginine:HCl (U-13C6, 99\%; U-15N4, 99\%) & Cambridge Isotope Laboratories \\
\hline Monoclonal anti-FLAG antibody (mouse) & Sigma \\
\hline NADH & Roche \\
\hline Ni-NTA agarose & Qiagen \\
\hline Oligonucleotides & Metabion \\
\hline Peroxidase anti-peroxidase, antibody (PAP) & Sigma \\
\hline PEG-4000 & Applichem \\
\hline Peptone, yeast extract, YNB w/o aa & $\mathrm{BD}$ \\
\hline ProteinA-seharose & GE- Healthcare \\
\hline Streptactin Sepharose & IBA \\
\hline SDS marker broad range & Biorad \\
\hline$\left[{ }^{35} \mathrm{~S}\right]$-methionine & Hartmann Analytics \\
\hline
\end{tabular}




\subsection{Cultivation of yeast}

\subsubsection{Growth condition and media}

In general, yeast (Saccharomyces cerevisiae) cells were grown in rich liquid media at $30{ }^{\circ} \mathrm{C}$ with agitation $(160-220 \mathrm{rpm})$. Synthetic medium was used to select for genetic markers and for growth of strains containing plasmids. Compositions of the different media are listed in table 4 . For solid media plates, 2.5\% agar was added prior to sterilization and preparation of the plates. Yeast cells were streaked onto appropriate solid media plates from cryo stocks and grown for $2-3$ days at $30{ }^{\circ} \mathrm{C}$. For liquid cultures, yeast cells were inoculated with biomass picked from solid media or from a pre-culture (1:10 or 1:20). Cell density was monitored by measuring optical density at $600 \mathrm{~nm}$ $\left(\mathrm{OD}_{600}\right)$. All media and solutions used in handling of yeast were either autoclaved or filter-sterilized.

\subsubsection{Yeast cryo stocks}

For yeast stock preservation, yeast cells were picked from solid media, resuspended in $2 x$ YPAD, containing $20 \%$ glycerol and stored at $-80{ }^{\circ} \mathrm{C}$.

\subsubsection{Growth test of yeast strains}

To assess the growth of yeast strains, serial 10 -fold dilutions of an overnight yeast preculture were spotted on solid media, supplemented with different carbon sources. Plates were incubated for 2-5 days at different temperatures. Subsequently, plates were scanned (Epson Perfection V750 Pro) for documentation of growth.

\subsubsection{Generation of $\boldsymbol{r h o}^{0}$ yeast strains}

Yeast cells were grown in YPD media, containing $25 \mu \mathrm{g} / \mathrm{ml}$ ethidiumbromide (EtBr) and diluted (1:60) in fresh YPD with EtBr every day. After 3 days, cells were plated on YPD solid media plates without EtBr, incubated for 2 days and single colonies were picked (modified from Simon \& Faye 1984). The absence of mitochondrial DNA was verified by the absence of growth on glycerol plates (non-fermentable carbon source) and the absence of various mitochondrially encoded proteins, assessed by Western blot analysis of whole cell extracts. 


\subsection{Cultivation of $E$. coli}

In general, E. coli cells were grown at $37^{\circ} \mathrm{C}$ in LB medium, supplemented with $100 \mathrm{mg} / \mathrm{l}$ ampicillin, with shaking for selection of plasmids carrying an $\mathrm{Amp}^{\mathrm{R}}$ marker. For solid media plates, $1.5 \%$ agar was added prior to sterilization and preparation of the LBplates. For liquid cultures, E. coli cells were inoculated with biomass picked from solid media or from a pre-culture (1:100 to $1: 1000)$. Cell density was monitored by measuring optical density at $600 \mathrm{~nm}\left(\mathrm{OD}_{600}\right)$. For stock preservation, $1 \mathrm{ml}$ liquid E. coli culture was mixed with $200 \mu \mathrm{l}$ of $80 \%$ glycerol and stored at $-80{ }^{\circ} \mathrm{C}$.

\subsection{Molecular biology methods}

\subsubsection{Amplification of DNA fragments by PCR}

DNA fragments were amplified by PCR using KOD Hot Start DNA polymerase (Novagen) according to the manufacturers specifications in $50 \mu \mathrm{l}$ scale (for standard reactions). 100 ng of yeast genomic DNA or $10 \mathrm{ng}$ of plasmid DNA were used as template. Cycling conditions were adjusted to the length of the fragment and melting temperature of the primer pair.

\subsubsection{Purification of plasmids}

Plasmids were purified from E. coli using Wizard ${ }^{\circledR}$ Plus SV Minipreps DNA Purification System (Promega), according to the manufacturers specifications. Following the purification, concentration of the nucleic acid solution was measured. Plasmids were stored in $\mathrm{dH}_{2} \mathrm{O}$ at $-20^{\circ} \mathrm{C}$. 


\subsubsection{Preparation of yeast genomic DNA}

Yeast cells were inoculated in YPGal and grown overnight. After determination of $\mathrm{OD}_{600}$, $2.5 \mathrm{OD}_{600}$ were isolated by centrifugation ( $2 \mathrm{~min}, 20000 \mathrm{xg}$, at RT). The supernatant was discarded, the pellet was resuspended in $150 \mu$ solution A (50 mM Tris/HCl (pH 7.4), $10 \mathrm{mM}$ EDTA, 0,3\% $\beta$-mercaptoethanol, $0.5 \mathrm{mg} / \mathrm{ml}$ zymolyase) and incubated at $37{ }^{\circ} \mathrm{C}$ for $1 \mathrm{~h}$ on a thermomixer (350 rpm). After addition of SDS to a final concentration of $1 \%, 0.5$ volumes of $8 \mathrm{M}$ ammoniumacetate were added, mixed thoroughly and incubated at $-20^{\circ} \mathrm{C}$ for 15 minutes. The lysate was cleared by centrifugation $\left(14000 \mathrm{rpm}, 15 \mathrm{~min}, 4^{\circ} \mathrm{C}\right.$ ) and $180 \mu \mathrm{l}$ of the supernatant were taken and DNA was precipitated by addition of $120 \mu \mathrm{l}$ isopropanol. After centrifugation $(14000 \mathrm{rpm}, 15 \mathrm{~min}$, $4{ }^{\circ} \mathrm{C}$ ) the pellet was washed with $70 \%$ EtOH once and dried. After resuspension in appropriate volume of TE buffer, the yeast genomic DNA was stored at $-20{ }^{\circ} \mathrm{C}$.

\subsubsection{DNA electrophoresis}

DNA fragments were separated by horizontal agarose gel electrophoresis. 1\% agarose gels were prepared in TAE buffer and supplemented with ethidiumbromide. DNA samples were mixed with loading dye (4x stock: $40 \%$ saccharose, 1\% OrangeG). Electrophoresis was performed in TAE buffer for 20-30 min at $100 \mathrm{~V}$. GeneRuler DNA Ladder Mix (Fermentas) was used as a standard. Separation of the DNA fragments was documented using a UV-transilluminator.

\subsubsection{Determination of nucleic acid concentrations}

DNA and RNA concentrations were measured with NanoVue spectrometer (GE Healthcare) at $260 \mathrm{~nm}$. One $\mathrm{OD}_{260}$ was assumed to correspond to $50 \mu \mathrm{g} / \mathrm{ml}$ for dsDNA and to $40 \mu \mathrm{g} / \mathrm{ml}$ for RNA.

\subsubsection{Sequencing of DNA}

Sequencing of DNA was performed by GATC Biotech (Konstanz). Sequences obtained were viewed and compared using GeniousPro (Version 5.3.6, Biomatters). 


\subsubsection{Site-directed mutagenesis of plasmids}

Site-directed mutagenesis was performed using the QuickChange® Site-Directed Mutagenesis Kit (Stratagene/Agilent) according to the manufacturers specifications. To mutate a single amino acid in COX15, $30 \mathrm{ng}$ of COX15 ${ }^{\mathrm{WT}}$, cloned into pRS416 was used as template. Overlapping primers were designed with the desired mutation in the middle of each primer (forward: 5'- CAG TTG GTC ATG AGG ACA TGT GCG TAC GTT G, CAT replaced by ATG; reverse: 5'- C ACA TGT CCT CAT GAC CAA CTG AAC TGT AAC, ATG replaced by CAT). Cycling conditions: initial denaturation $\left(95^{\circ} \mathrm{C}, 30 \mathrm{sec}\right)$, followed by 20 cycles of denaturation $\left(95^{\circ} \mathrm{C}, 30 \mathrm{sec}\right)$, annealing $\left(55^{\circ} \mathrm{C}, 1 \mathrm{~min}\right)$ and amplification $\left(68^{\circ} \mathrm{C}, 8 \mathrm{~min}\right)$. To digest methylated, non mutated parental DNA template, the reaction was treated with $D p n I$ at $37^{\circ} \mathrm{C}$ for $1 \mathrm{~h}$. The mutated plasmid was transformed into XL1Blue supercompetent cells, supplied with the kit, according to the general E. coli transformation protocol (see section 2.4.9) with the following modifications: cells were left on ice for $30 \mathrm{~min}$ prior to transformation and heat shock was performed for $45 \mathrm{sec}$ instead of $2 \mathrm{~min}$. The mutagenesis was verified by sequencing.

\subsubsection{Chromosomal deletion and tagging of yeast genes}

Chromosomal deletions as well as tagged versions of yeast genes were generated by introduction of the kITRP1, HIS3MX6 or natNT2 cassettes using PCR based strategies (Knop et al. 1999; Janke et al. 2004). Cox15 ${ }^{\mathrm{FLAG}}$ was generated using pYM2.1 vector (D. Mick, Rehling lab). Mss51 ${ }^{\text {SF }}$ was generated using a modified pYM2.1 vector, kindly provided by J. Melin (Rehling lab). Briefly, the streptavidin-FLAG tag from pESGIBA_168 (cut with XhoI and BglII) was ligated into pYM2.1, cut with SalI and BglII (Alkhaja et al. 2012).

Yeast strains were transformed with PCR-amplified integration-cassettes using the lithium acetate method, as described in section 2.4.10. Integration into the genome was confirmed by PCR or Western blot analysis of the target protein. The strains generated in this study are listed in table 5. 


\subsubsection{Transformation of $E$. coli}

$100 \mu \mathrm{l}$ of rubidium chloride $(\mathrm{RuCl})$ competent E. coli cells were thawed on ice and incubated with $50 \mathrm{ng}$ plasmid for $15 \mathrm{~min}$ on ice. The reaction was subjected to heat shock at $42{ }^{\circ} \mathrm{C}$ for $2 \mathrm{~min}$ and subsequently cooled down on ice for $3 \mathrm{~min}$. $1 \mathrm{ml}$ of LB media, pre-warmed to $37^{\circ} \mathrm{C}$, was added and bacteria were allowed to grow for $1 \mathrm{~h}$ at $37^{\circ} \mathrm{C}$ with shaking. The transformed cells were centrifuged down $(10000 \mathrm{rpm}, 5 \mathrm{~min}$ at RT), resuspended in $200 \mu \mathrm{LB}$ and plated onto LB-Amp agar plates. Plates were incubated at $37^{\circ} \mathrm{C}$ over night until appearance of single colonies.

\subsubsection{Transformation of $S$. cerevisiae}

Yeast cells were transformed by the LiOAc method (Ito et al. 1983; Schiestl \& Gietz 1989; Gietz \& Schiestl 2007). Cells were inoculated on the day before transformation from a fresh agar plate into 2x YPAD and grown overnight. On the next day, the main culture was inoculated with the pre-culture to an $\mathrm{OD}_{600}$ of 0.5 and allowed to double twice. At $\mathrm{OD}_{600}=2$, the yeast cells were harvested $\left(4000 \mathrm{rpm}, 3 \mathrm{~min}, 18^{\circ} \mathrm{C}\right)$, washed once with 0.5 volumes of sterile water and once with 0.5 volumes of transformation buffer (0.1 M LiOAc, 0.1 mM EDTA, 5 mM Tris/HCl pH 8.0). The cells were subsequently resuspended in transformation buffer (1/50 volumes of the original culture) and aliquoted to $100 \mu \mathrm{l}$ per reaction. To each reaction, $1 / 10$ volume of carrier DNA $\left(10 \mathrm{mg} / \mathrm{ml}\right.$ herring sperm DNA, boiled at $95^{\circ} \mathrm{C}$ for $10 \mathrm{~min}$ prior to addition) and the target DNA (1-2 $\mu \mathrm{g}$ PCR product/ $30 \mathrm{ng}$ plasmid DNA) was added. For a control reaction, the target DNA was replaced by the same volume of sterile water. After addition of $600 \mu \mathrm{l} 40 \%$ PEG-4000 in transformation buffer, the mixture was incubated at $30{ }^{\circ} \mathrm{C}$ for $30 \mathrm{~min}$. Heat shock was performed at $42{ }^{\circ} \mathrm{C}$ for $15 \mathrm{~min}$ after addition of $68 \mu \mathrm{l}$ DMSO. Cells were harvested by centrifugation (2000 rpm, 2 min at RT), resuspended in $200 \mu \mathrm{l} 1 \mathrm{M}$ sorbitol and plated onto appropriate selective media plates under sterile conditions. Plates were incubated at $30^{\circ} \mathrm{C}$ until appearance of colonies (usually 3 days), then single colonies were picked and re-streaked onto selective media plates. All solutions used for this procedure were either autoclaved or filter-sterilized. 


\subsection{Biochemical methods}

\subsubsection{SDS-PAGE}

SDS-PAGE was performed to separate proteins according to their molecular weight under denaturing conditions by one-dimensional SDS-Polyacrylamide gel electrophoresis (SDS-PAGE), originally developed by Laemmli (1970). Gels used in this study contained $0.1 \%$ SDS and $4 \%$ acrylamide for the stacking gel and $12.5-17 \%$ acrylamide for the separating gel (depending on the size of proteins to separate). Acrylamide stock solution used to prepare gels contained $30 \%$ acrylamide/bisacrylamide (37.5: 1). Samples were mixed with SDS loading buffer and incubated at $25^{\circ} \mathrm{C}$ for $15 \mathrm{~min}$ prior to loading. Electrophoresis was performed in custom-made midi gel systems $(250 \mathrm{~V}, 30 \mathrm{~mA}$ per gel). As a standard, SDS-PAGE standard Broad Range (Biorad) was used.

\subsubsection{Urea-SDS-PAGE}

For better separation and resolution in the low molecular range (below $10 \mathrm{kDa}$ ), SDS-PAGE gels, containing urea were used. Handling and electrophoresis conditions were basically identical to SDS-PAGE gels. The resolving gel contained $5.4 \mathrm{M}$ urea, $0.09 \%$ SDS, $17.5 \%$ acrylamide, $0.23 \%$ bisacrylamide. The stacking gel contained $5.5 \%$ acrylamide $/ 0.07 \%$ bisacrylamide, $0.12 \%$ SDS, $3.6 \mathrm{M}$ urea. The running buffer was composed of $50 \mathrm{mM}$ Tris, $192 \mathrm{mM}$ glycine, 0.1\% SDS. Electrophoresis was performed for 4-5 h.

\subsubsection{BN-PAGE}

For separation of native protein complexes, Blue Native polyacrylamide gel electrophoresis (BN-PAGE) was basically performed as described earlier (Schägger \& Jagow 1991; Wittig et al. 2006). Prior to the gel run, mitochondria were solubilized in digitonin solubilization buffer, containing $1 \%$ digitonin, at a concentration of $1 \mathrm{mg} / \mathrm{ml}$. To ensure proper solubilization, mitochondria were re-isolated by centrifugation (14000 rpm, $10 \mathrm{~min}, 4^{\circ} \mathrm{C}$ ) and resuspended by pipetting up and down 12 times using a small tip. After incubation of the samples for $30 \mathrm{~min}$ on ice, insoluble material was removed by centrifugation $\left(14000 \mathrm{rpm}, 15 \mathrm{~min}, 4^{\circ} \mathrm{C}\right)$. The supernatant was mixed with BN loading dye to a final concentration of $1 \mathrm{x}$. 
$4-10 \%$ or $4-13 \%$ gradient gels with $4 \%$ stacking gel in a Hoefer gel system, equipped with a cooling device to allow electrophoresis at $4{ }^{\circ} \mathrm{C}$ were used in this study. All buffers were precooled to $4{ }^{\circ} \mathrm{C}$ and gel loading was performed in a cold room at $4{ }^{\circ} \mathrm{C}$. Electrophoresis was performed with anode buffer, containing Coomassie at $200 \mathrm{~V}$ and $15 \mathrm{~mA}$ per gel for $2 \mathrm{~h}$. Anode buffer was replaced by anode buffer without Coomassie and electrophoresis was continued at $600 \mathrm{~V}$ and $15 \mathrm{~mA}$ per gel (3-4 h). Replacement of the anode buffer was omitted if the gel was not intended for Western blotting. Following the gel run, the gel was either stained with Coomassie or subjected to Western blotting. As a standard, the HMW calibration kit (GE Healthcare) was used.

For analysis of steady state protein levels of structural subunits of respiratory chain complexes, 15-20 $\mu \mathrm{g}$ of mitochondria were loaded per lane. For analysis of steady state levels of less abundant proteins (e.g. COX assembly factors), 50-70 $\mu$ g of mitochondria were loaded per lane. For analysis of elution of protein complex isolations, the amount loaded per lane varied, but usually corresponded to a starting material between 250-500 $\mu$ g of mitochondria.

\subsubsection{Determination of protein concentrations}

Protein concentrations were determined by Bradford analysis (Bradford 1976) using Roti-quant (Roth) according to the manufacturers specifications with bovine IgG (Biorad) as a standard. Absorbance of the protein solutions was measured using a BioPhotometer (Eppendorf) at $595 \mathrm{~nm}$. Protein concentrations were calculated based on the IgG standard. 


\subsubsection{Yeast whole cell extracts}

For analysis of proteins on a whole cell level, whole cell extracts were prepared essentially as described earlier (Yaffe \& Schatz 1984). In detail, yeast cells were grown in YPGal overnight. After determination of $\mathrm{OD}_{600}, 2 \mathrm{OD}_{600}$ were isolated by centrifugation (14000 rpm, 1 min at RT). The cells were resuspended in $\mathrm{dH}_{2} \mathrm{O}, 148 \mu \mathrm{l}$ $2 \mathrm{M} \mathrm{NaOH}$ and $12 \mu \mathrm{l} \beta$-mercaptoethanol were added and the mixture incubated on ice for $10 \mathrm{~min}$. Trichloracetic acid (TCA) was added to a final concentration of $7 \%$ and incubated on ice for $10 \mathrm{~min}$. Precipitated material was pelleted by centrifugation $(2 \mathrm{~min}$, $14000 \mathrm{rpm}, 4{ }^{\circ} \mathrm{C}$ ) and the pellet resuspended in SDS sample buffer. The $\mathrm{pH}$ of the whole cell lysate was adjusted to a neutral pH (indicated by the blue colour of the SDS loading buffer) by titration with $1 \mathrm{M}$ Tris ( $\mathrm{pH}$ 11.5). Before the samples were subjected to SDSPAGE, debris was removed by centrifugation (14000 rpm, 1 min at RT).

\subsubsection{Western Blotting}

After separation by polyacrylamide gelelectrophoresis, proteins were transferred and immobilized onto PVDF membranes (Millipore) using semidry blotting chambers (Peqlab). PVDF membranes were pre-activated by short incubation in MeOH. The membrane was subsequently soaked in blotting buffer, together with gels and Whatman paper. After assembly of the blotting sandwich, transfer was performed at $25 \mathrm{~V}$ and 250 $\mathrm{mA}$ for $90 \mathrm{~min}$ (following SDS-PAGE) or for $120 \mathrm{~min}$ (following BN-PAGE and Urea-SDSPAGE).

\subsubsection{Coomassie staining}

Proteins on PVDF membranes or in polyacrylamide gels were stained with Coomassie Brilliant Blue R-250 for 2 min (membranes) or for $1 \mathrm{~h}$ (gels). After staining, marker bands were labeled on the membrane and membranes were destained for $5 \mathrm{~min}$, gels for up to $1 \mathrm{~h}$. Additionally, PVDF membranes were completely destained in $\mathrm{MeOH}$. 


\subsubsection{Immunodecoration of proteins on PVDF membranes}

Destained PVDF membranes were blocked using 5\% milk powder in TBS-T for $1 \mathrm{~h}$ at room temperature or over night at $4{ }^{\circ} \mathrm{C}$. Following blocking of non-specific interaction sites, membranes were incubated in primary antibody solution (in 5\% milk in TBS-T likewise) for $1 \mathrm{~h}$, washed 3 times for $10 \mathrm{~min}$ in fresh TBS-T. Subsequently, membranes were incubated in appropriate secondary antibody, coupled to HRP (solution prepared 1:5000-1:10000 in 5\% milk in TBS-T) for $1 \mathrm{~h}$, followed by 3 washes for $15 \mathrm{~min}$ in TBS-T. All incubation and washing steps were performed at room temperature and under agitation. For detection and visualization of antibody-protein complexes, enhanced chemiluminescence reagent (GE Healthcare) were added to the membranes and signals were detected on medical X-ray films (Foma).

\subsubsection{Detection of radiolabeled proteins by autoradiography}

For detection of radiolabeled proteins, SDS- or BN-PAGE gels were dried in a vacuum gel drier at $65^{\circ} \mathrm{C}$ for $2 \mathrm{~h}$. Dried gels were exposed to Storage Phosphor Screens (GE Healthcare) for a few hours up to several days and signals were digitized using Storm820 scanner (GE Healthcare).

\subsubsection{Steady state protein analyses}

For steady state analysis of mitochondrial proteins by SDS-PAGE, mitochondria were diluted to different concentrations so that identical volumes could be loaded per gel lane. Following SDS-PAGE, various mitochondrial proteins from different compartments were analysed by Western blotting.

For analysis of steady state protein levels by BN-PAGE, 15-20 $\mu \mathrm{g}$ of solubilized mitochondria were loaded per lane, if structural subunits of respiratory chain complexes were assessed. For analysis of steady state levels of less abundant proteins (e.g. COX assembly factors), 50-70 $\mu$ g of mitochondria were loaded per lane. 


\subsection{Isolation of yeast mitochondria}

Mitochondria were prepared essentially as described previously (Meisinger et al. 2006). In brief, yeast cells were grown in appropriate liquid media at $30{ }^{\circ} \mathrm{C}$ to $\mathrm{OD}_{600}=2$. Cells were harvested (4700 rpm, $15 \mathrm{~min}, 18{ }^{\circ} \mathrm{C}$ ), washed with $\mathrm{H}_{2} \mathrm{O}$, resuspended in $2 \mathrm{ml} / \mathrm{g}$ wet cell weight of DTT buffer (100 mM Tris $/ \mathrm{H}_{2} \mathrm{SO}_{4}, \mathrm{pH}$ 9.4, $10 \mathrm{mM}$ DTT) and incubated under mild agitation $(90 \mathrm{rpm})$ at $30^{\circ} \mathrm{C}$ for $20 \mathrm{~min}$. Cells were pelleted $(4000 \mathrm{rpm}, 8 \mathrm{~min}$, $18^{\circ} \mathrm{C}$ ), washed with zymolyase-buffer (1.2 M Sorbitol, $20 \mathrm{mM} \mathrm{KPi}, \mathrm{pH} 7.4$ ), resuspended in $7 \mathrm{ml} / \mathrm{g}$ wet cell weight of zymolyase-buffer, supplied with $4 \mathrm{mg}$ zymolyase/g wet cell weight to digest cell walls. After incubation ( $\left.90 \mathrm{rpm}, 60 \mathrm{~min}, 30^{\circ} \mathrm{C}\right)$, spheroplasts were isolated (3000 rpm, $\left.10 \mathrm{~min}, 18^{\circ} \mathrm{C}\right)$, washed with zymolyase-buffer and subsequently resuspended in chilled homogenisation buffer (0.6 M sorbitol, $10 \mathrm{mM}$ Tris/HCl, pH 7.4, 1 mM EDTA, 0.1\% BSA, 1 mM PMSF). After homogenization with a douncer (12 times, $650 \mathrm{rpm}$ ), cell debris was removed by centrifugation $\left(3000 \mathrm{rpm}, 5 \mathrm{~min}, 4^{\circ} \mathrm{C}\right.$, followed by $4000 \mathrm{rpm}, 10 \mathrm{~min}, 4^{\circ} \mathrm{C}$ ). Mitochondria (crude membrane fraction) were obtained by centrifugation (12000 rpm, $15 \mathrm{~min}, 4^{\circ} \mathrm{C}$ ). This pellet was washed once and finally resuspended in SEM. Protein concentration was determined by Bradford analysis, mitochondria were snap-frozen in liquid $\mathrm{N}_{2}$ at a concentration of $10 \mathrm{mg} / \mathrm{ml}$ in SEM and stored at $-80^{\circ} \mathrm{C}$ until further processing. 


\subsection{Purification of protein complexes}

\subsubsection{IgG-Chromatography}

IgG-chromatography was performed essentially as described previously (Rehling et al. 2003). In detail, for native complex isolation, human IgGs (Sigma-Aldrich) were coupled to $\mathrm{CNBr}$-activated sepharose (GE Healthcare) according to manufacturers specifications. Mitochondria from wild type (WT) and ProteinA tagged strains were solubilized on ice in $1 \mathrm{ml} / \mathrm{mg}$ solubilisation buffer (containing 1\% digitonin) for $30 \mathrm{~min}$. After removal of unsolubilized material by centrifugation ( $15 \mathrm{~min}, 20000 \mathrm{~g}, 4{ }^{\circ} \mathrm{C}$ ), a sample was taken and the mitochondrial extract applied to IgG-sepharose for $2 \mathrm{~h}$ at $4{ }^{\circ} \mathrm{C}$ with mild agitation. After extensive washing with wash buffer $(20 \mathrm{mM}$ Tris/HCl (pH 7.4), $0.1 \mathrm{M}$ $\mathrm{NaCl}, 10 \%$ glycerol, 5 mM EDTA, 2 mM PMSF, 0.3\% digitonin), AcTEV (Tobacco Etch Virus) protease (Invitrogen, $10 \mathrm{U} / \mu \mathrm{l}$ ) was added and incubated over night at $4{ }^{\circ} \mathrm{C}$ to cleave ProteinA tagged protein off the sepharose. TEV protease, carrying a polyhistidine, was removed by addition of Ni-NTA (Qiagen), pre-equilibrated with wash buffer. The cleaved native complexes were eluted, mixed with appropriate loading buffer and further analysed by SDS- or BN-PAGE. Samples that were only analysed by SDS-PAGE were directly eluted with SDS loading buffer instead.

\subsubsection{Crosslinking of antibodies to sepharose}

Coa3/ Coa1 or, as a control, Ylh47-specific antisera (raised in rabbit) was bound to ProteinA-sepharose (GE Healthcare) in $0.1 \mathrm{M}$ potassium phosphate buffer, (pH 7.4) for 1 $\mathrm{h}$ at room temperature and subsequently cross-linked with $5 \mathrm{mg} / \mathrm{ml}$ dimethyl pimelimidate (DMP) solution in $0.1 \mathrm{M}$ sodium borate $(\mathrm{pH} 9.0$ ) for $30 \mathrm{~min}$ at room temperature. The crosslinker was quenched with $1 \mathrm{M}$ Tris/HCl (pH 7.4). Beads were washed with TBS several times and stored in TBS, supplemented with 2 mM sodium azide at $4{ }^{\circ} \mathrm{C}$ until use for co-immunoprecipitation. After co-immunoprecipitation, beads were washed three times with acetate buffer ( $\mathrm{pH} 3.4)$, three times with TBS, stored as described and were reused up to 7 times. 


\subsubsection{Co-immunoprecipitation}

Co-immunoprecipitation was performed as described (Hutu et al. 2008; Mick et al. 2010), with minor changes. After mitochondria were solubilized and the lysate cleared as described above, an input sample was taken. The mitochondrial lysate was split and bound to control and Coa3 or Coa1 coupled sepharose for $90 \mathrm{~min}$ at $4{ }^{\circ} \mathrm{C}$ under mild agitation. After washing with digitonin wash buffer as described in section 2.7.1, proteins were eluted with $0.1 \mathrm{M}$ Glycin ( $\mathrm{pH} 2.8$ ) and immediately neutralized with $1 \mathrm{M}$ Tris (pH 11.5). Eluates and the total sample were analysed by SDS-PAGE and Western blotting.

\subsubsection{FLAG-isolation}

For isolation of native complexes via FLAG-tagged proteins, mitochondria were solubilized in digitonin solubilization buffer as described above. An input sample was taken. Mitochonrial lysate was bound to pre-equilibrated FLAG-agarose (Sigma) for $1 \mathrm{~h}$ at $4{ }^{\circ} \mathrm{C}$. The unbound material was removed and the agarose washed 10 times with cold digitonin wash buffer. Bound protein complexes were eluted by incubation with FLAGpeptide (200 $\mu \mathrm{g} / \mathrm{ml}$ in digitonin wash buffer) for $10 \mathrm{~min}$ at $4{ }^{\circ} \mathrm{C}$ and shaking (900 rpm). Subsequently, samples were mixed with appropriate loading dye and analysed by BNor SDS-PAGE, followed by Western blotting. 


\subsubsection{Strep-Isolation}

For isolation of native complexes via Strep-tagged proteins, mitochondria were solubilized in digitonin solubilization buffer and cleared as described above. In input sample was taken. Mitochondrial lysate was bound to pre-equilibrated Strep-Tactin ${ }^{\circledR}$ Sepharose (IBA) for $1 \mathrm{~h}$ at $4{ }^{\circ} \mathrm{C}$. The unbound material was removed and the sepharose washed extensively with cold digitonin wash buffer. Bound protein complexes were eluted by incubation with elution buffer ( $5 \mathrm{mM}$ desthiobiotin in digitonin wash buffer) for $15 \mathrm{~min}$ at $12^{\circ} \mathrm{C}$ and shaking ( $900 \mathrm{rpm}$ ). For further analysis, samples were either mixed with appropriate loading dye and subjected to BN- or SDS-PAGE followed by Western blotting or samples were precipitated, using TCA and subjected to mass spectrometry. In brief, TCA (to a final concentration of 15\%) and sodium desoxycholate (to $0.0125 \%$ final concentration) was added and samples were incubated for $30 \mathrm{~min}$ on ice. After centrifugation ( $\left.14000 \mathrm{rpm}, 30 \mathrm{~min}, 4^{\circ} \mathrm{C}\right)$, the pellet was washed twice using ice-cold acetone, dried and resuspended in $20 \mathrm{mM}$ Tris/ $\mathrm{HCl}(\mathrm{pH} 7.4), 100 \mathrm{mM} \mathrm{NaCl}$, $10 \%$ glycerol, 5 mM EDTA. 


\subsection{In vitro import}

\subsubsection{In vitro labeling of precursor proteins}

\subsubsection{In-vitro transcription}

RNA was transcribed from DNA template using SP6 RNA polymerase. PCR products, amplified from yeast genomic DNA, served as templates. The SP6 promotor was introduced during PCR with the primers. The purified PCR product was used for in vitro transcription using mMESSAGE mMACHINE® SP6 Kit (Ambion) according to the manufacturers specification. Resulting RNA was purified using MEGAclear kit (Ambion) and stored in $\mathrm{dH}_{2} \mathrm{O}$ at $-80{ }^{\circ} \mathrm{C}$.

\subsubsection{In-vitro translation}

Proteins were labeled with $\left[{ }^{35} \mathrm{~S}\right]$-methionine by in vitro translation of purified RNA using Flexi Rabbit Reticulocyte Lysate System (Promega ). For translation of Cox13 RNA, $33 \mu \mathrm{l}$ of lysate was mixed with $1 \mu \mathrm{l}$ of amino acid mix (-Met), $1 \mu \mathrm{g}$ RNA, $1,4 \mu \mathrm{l}$ potassium chloride (2.5 M), $1 \mu \mathrm{l}$ magnesium acetate $\left(25 \mathrm{mM}\right.$ ) and $\mathrm{H}_{2} \mathrm{O}$ (up to $45 \mu \mathrm{l}$ ). After short pre-warming at $30^{\circ} \mathrm{C}$, the translation reaction was started by addition of $5 \mu \mathrm{l}\left[{ }^{35} \mathrm{~S}\right]$-methionine $(=50 \mu \mathrm{Ci})$. Following in vitro translation for $90 \mathrm{~min}$ at $30^{\circ} \mathrm{C}$, the reaction was stopped by addition of excess methionine $(20 \mathrm{mM})$ and sucrose $(250 \mathrm{mM})$ and placed on ice. Lysate was frozen in liquid nitrogen and stored at $-80{ }^{\circ} \mathrm{C}$.

\subsubsection{Coupled in vitro transcription/translation}

Coupled in vitro transcription/translation was performed with the TNT SP6 Quick Coupled Transcription/Translation System (Promega). The open reading frame of COX5A was cloned into pGEM4Z under control of the SP6 promotor and the plasmid was isolated. $50 \mu \mathrm{l}$ lysate was mixed with $0.5 \mu \mathrm{g}$ plasmid DNA and pre-warmed for 2 $\min$ at $30^{\circ} \mathrm{C}$. The reaction was started by addition of $5 \mu \mathrm{l}\left[{ }^{35} \mathrm{~S}\right]$-methionine $(=50 \mu \mathrm{Ci})$, incubated for $90 \mathrm{~min}$ at $30^{\circ} \mathrm{C}$ and stopped by addition of sucrose $(250 \mathrm{mM})$ and placed on ice. Lysate was frozen in liquid nitrogen and stored at $-80^{\circ} \mathrm{C}$. 


\subsubsection{In vitro import into isolated mitochondria}

Radiolabeled precursor proteins were in vitro imported into energized mitochondria as described previously (Wiedemann et al. 2006). For import, isolated yeast mitochondria were resuspended in import buffer $(250 \mathrm{mM}$ sucrose, $10 \mathrm{mM} \mathrm{MOPS} / \mathrm{KOH}, \mathrm{pH} 7.2$, $80 \mathrm{mM} \mathrm{KCl}, 2 \mathrm{mM} \mathrm{KH}_{2} \mathrm{PO}_{4}, 5 \mathrm{mM} \mathrm{MgCl}_{2}, 5 \mathrm{mM}$ methionine, 3\% BSA, $2 \mathrm{mM}$ NADH, $2 \mathrm{mM}$ ATP, $5 \mathrm{mM}$ creatine phosphate, $0.1 \mathrm{mg} / \mathrm{ml}$ creatine kinase) at $0.5 \mathrm{mg} / \mathrm{ml}$. After addition of $5-10 \%$ lysate, import was performed at $25{ }^{\circ} \mathrm{C}$ for indicated times. Import was stopped by addition of $1 \%$ AVO mix $(2.5 \mathrm{mM}$ antimycin A, $0.25 \mathrm{mM}$ valinomycin, $5 \mathrm{mM}$ oligomycin in ethanol). In negative controls, samples were treated with AVO prior to import. If not indicated otherwise, unimported precursor was digested by addition of proteinase $\mathrm{K}(50 \mu \mathrm{g} / \mathrm{ml})$ for $10 \mathrm{~min}$ on ice. Digestion was subsequently inhibited by PMSF (4 mM). After in vitro import, mitochondria were reisolated, washed with SEM buffer and processed for SDS- or BN-PAGE analysis, followed by detection of radiolabeled proteins by digital autoradiography.

\subsection{Stable isotope labeling with amino acids in cell culture (SILAC)}

For SILAC, yeast strains auxotroph for lysine and arginine were used. As YPH499 is already auxotroph for lysine, $\arg 4$, one gene in the arginine biosynthesis, was deleted in coa1s and in coa1s, expressing Mss51-StrepFLAG to generate the strains for SILAC labeling. Both resulting strains were grown both on media containing heavy amino acids as well as on media containing normal (light) amino acids in order to perform the experiment in forward and reverse manner. For the forward experiment, the tagged strains was grown on media, containing heavy amino acids; the untagged control strains on media containing light amino acids and vice versa for the reverse experiment. Yeast cells were grown in SILAC media (see table 4), supplied with $2 \%$ galactose at $30{ }^{\circ} \mathrm{C}$ as described above. High concentration of proline $(200 \mathrm{mg} / \mathrm{l})$ was used to prevent the conversion of (heavy) arginine into proline. Mitochondrial preparation followed the procedure described in section 2.6. Prior to complex isolation, mitochondria from cells grown on light and heavy media were mixed in a 1:1 ratio and solubilized together. 


\subsection{Mass spectrometry and data analysis}

Mass spectrometry, including in-gel digestion, data analysis and visualization was performed in the Bioanalytical Mass spectrometry Group of Prof. Urlaub at the MPI BPC (Göttingen), mainly by Monika Raabe and Dr. Miroslav Nikolov.

\subsubsection{Mass spectrometry of native protein complexes}

For Shy1 interactome analysis, eluted proteins were mixed with 10x BN loading dye and separated on a 4-13\% gradient BN-PAGE gel. Directly following the gel run, the corresponding gel lane was cut into 23 gel slices of equal size. Prior in-gel digestion, gel slices were washed three times in $5 \mathrm{mM}$ ammonium bicarbonate/ $50 \%$ acetonitrile buffer $(\mathrm{pH}$ 8.0) and proteins therein in-gel digested with trypsin (Promega) as described (Shevchenko et al. 2006). Chymotrypsin was used as protease instead of trypsin in replicate experiments. Tryptic/chymotryptic peptides from each gel slice were analysed as described (Thakar et al. 2013) by nanoflow HPLC (Agilent 1100, Agilent Technologies) coupled to nanoelectrospray LTQ-Orbitrap XL mass spectrometer (Thermo Fischer Scientific).

The raw MS files from the mass spectrometer were analysed by MaxQuant and Andromeda using UniProt $S$. cerevisiae protein database (version 29.11.11). Results from MaxQuant were analysed and visualized with R as described (Nikolov et al. 2011). "Match between runs" option in MaxQuant was selected in replicate analysis to detect low abundant/hydrophobic peptides of Cox15.

\subsubsection{Mass spectrometry of SILAC samples}

For SILAC interactome analysis, samples and data were processed and analysed as described ins section 2.10.1 with some modifications: eluted proteins were separated on $4-12 \%$ gradient SDS-PAGE gels (Invitrogen) and stained with Colloidal Coomassie Blue. Each gel lane was cut into 23 equal gel slices and proteins therein were in-gel digested with trypsin (Promega), omitting additional wash steps described above. Tryptic peptides from each gel slice were analysed by nanoflow HPLC coupled to nanoelectrospray LTQ-Orbitrap XL mass spectrometer. Raw MS data was analysed with MaxQuant and Andromeda assuming standard SILAC workflow with mixed heavy- and light-labeled samples. 


\subsubsection{SILAC incorporation efficiency}

For calculation of SILAC incorporation efficiency, only heavy samples were analysed: whole cell extracts were separated by SDS-PAGE and stained with Colloidal Coomassie Blue. One band was randomly selected and cut out from the gel. Proteins in this gel piece were in-gel digested with trypsin (Promega), and analysed as described for SILAC interactome analysis (section 2.10.2). Raw MS data was analysed with MaxQuant and Andromeda assuming standard SILAC workflow with mixed heavy- and light-labeled samples. Standard MaxQuant settings were used, except for the Re-quantify option, which was disabled. The density functions of the resulting SILAC ratios for Arg- and Lyscontaining peptides were calculated and plotted separately and combined using R. The maximum value of each density function was used as incorporation efficiency. 


\section{RESULTS}

\subsection{Translational regulation and early steps of COX assembly}

\subsubsection{CoX assembly in Oxa1 linker mutants}

\subsubsection{Steady state protein levels are affected in 0xa1 linker mutants}

Oxa1 acts very early in the biogenesis of cytochrome $c$ oxidase as the insertase for the three mitochondria-encoded subunits of cytochrome $c$ oxidase (Hell et al. 2001). To fulfill this function, the Oxa1 protein contains two functional domains: a N-terminal insertase domain and a C-terminal ribosome binding domain (Bonnefoy et al. 2009; Jia et al. 2009). To test the importance of the close contact of the ribosome and the insertion site, Oxa1 versions with a linker of different length between the two domains were constructed (Keil et al. 2012). For the linkers regions, 100 or 200 residues of an intrinsically unstructured region on the basis of nucleofilament Nsp1 were inserted into Oxa1 (Keil et al. 2012). The constructs, expressed in yeast strains lacking the ribosome receptor Mba1, showed a length-dependent growth phenotype on non-fermentable media (YPG), as well as reduced oxygen consumption, indicating a deficiency in mitochondrial respiration (Keil et al. 2012). It is known that Oxa1 plays a crucial role in the biogenesis of cytochrome $c$ oxidase (Altamura et al. 1996). To assess the respiratory defect, yeast cells were grown on SGal-Trp at $30{ }^{\circ} \mathrm{C}$ and after mitochondria isolation, steady state levels of mitochondrial proteins were analysed by SDS- and BN-PAGE, followed by Western blotting (FIG 3.1). On SDS-PAGE, Oxa1 linker constructs were detected at the expected size and 0xa1 protein levels were comparable to wild-type

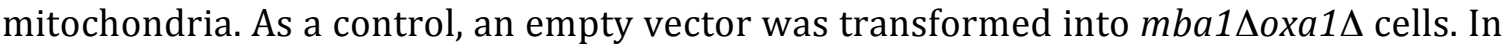
accordance with published data, Cox 1 and Cox 2 were undetectable in mitochondria isolated from this strain, due to proteolytic instability (Preuss et al. 2001). In

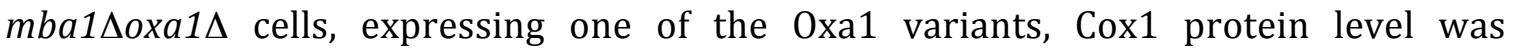
drastically reduced while Cox 2 showed only a moderate reduction. The nuclearencoded subunit Cox4, as well as cytochrome $c$ oxidase assembly factors (Coa1, Coa3) were unaffected. Furthermore, Atp5 (component of $\mathrm{F}_{1} \mathrm{~F}_{0}$-ATP-synthase) and the matrix protein Aco1 remained unaltered (FIG 3.1; A). 
To investigate the effects of $0 x a 1^{100}$ and $0 x a 1^{200}$ on mitochondrial respiratory chain complex formation, digitonin solubilized mitochondria prepared from the described strains were subjected to native gelelectrophoresis. This analysis revealed strongly reduced levels of supercomplexes. Mitochondria lacking Oxa1 and Mba1 contained almost no detectable supercomplexes.

Upon expression of wild type Oxa1, the different respiratory chain supercomplexes $\mathrm{III}_{2}$, $\mathrm{III}_{2} \mathrm{IV}, \mathrm{III}_{2} \mathrm{IV}_{2}, \mathrm{~V}$ and $\mathrm{V}_{2}$ were present. Upon expression of a linker variant of Oxa1, the supercomplexes containing cytochrome c oxidase ( $\mathrm{III}_{2} \mathrm{IV}$ and $\left.\mathrm{III}_{2} \mathrm{IV}_{2}\right)$ were severely reduced. Other OXPHOS complexes were not affected (FIG 3.1; B).

A

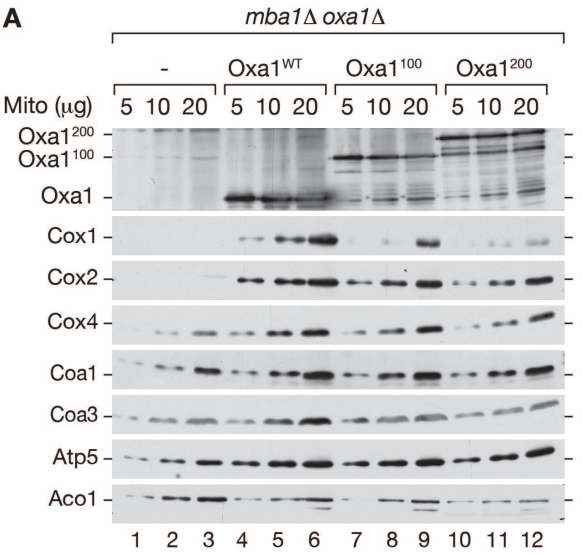

B

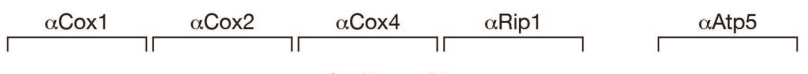

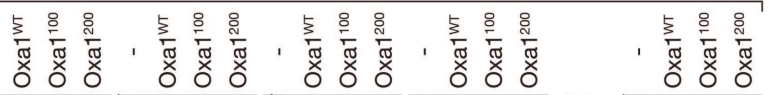

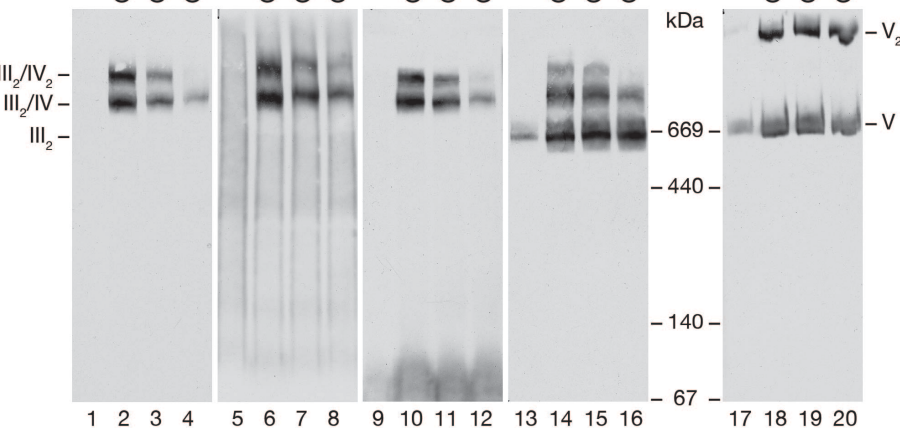

FIG 3.1 Analysis of steady state protein levels and OXPHOS complexes in Oxa1 linker mutants

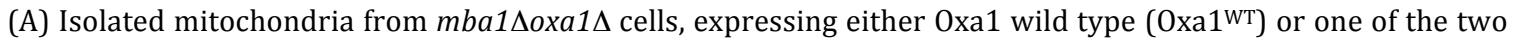
linker mutant version of Oxa1 (Oxa1100 / Oxa1200), were separated by SDS-PAGE, followed by Western blot analysis for various mitochondrial proteins. (B) Digitonin extracts of mitochondria were analysed by BN-PAGE and Western blotting for components of cytochrome $c$ oxidase (Cox1, Cox2, Cox4), the cytochrome $b c_{1}$ comlex (Rip1) or the ATPase (Atp5).

Thus, the growth phenotype is caused by reduced levels of cytochrome $c$ oxidase. Furthermore, we concluded from these results that insertion of a flexible linker into Oxa1 (in mutants lacking Mba1) interferes with the biogenesis or the stability of cytochrome $c$ oxidase. We could also show that this effect is more severe with increasing linker length. 


\subsubsection{Assembly of Cox5a/Cox13 is disturbed in 0xa1 mutants}

Oxa1 is required for membrane insertion of Cox2 (He \& Fox 1997). It was shown by our collaborators that processing of $\operatorname{Cox} 2$ was not affected in the linker mutants and therefore the defect in biogenesis of cytochrome $c$ oxidase was not due to defects in translation or maturation of newly synthesized Cox2. As the observed cytochrome $c$ oxidase defects could not be caused by reduced synthesis or disturbed membrane insertion, we asked whether further downstream events were affected in the linker mutants which would point to a role of Oxa1 in later steps of cytochrome $c$ oxidase assembly. To test this hypothesis, we imported two radiolabeled subunits of cytochrome $c$ oxidase (Cox5a and Cox13) into isolated mitochondria and followed their assembly into supercomplexes using BN-PAGE (FIG 3.2). We selected these two different subunits because Cox5a is known to assemble predominantly into COA complexes, whereas the late subunits, like Cox13, assemble directly into supercomplexes in association with the cytochrome $b c_{1}$ dimer (Brandner et al. 2005; Mick et al. 2007).
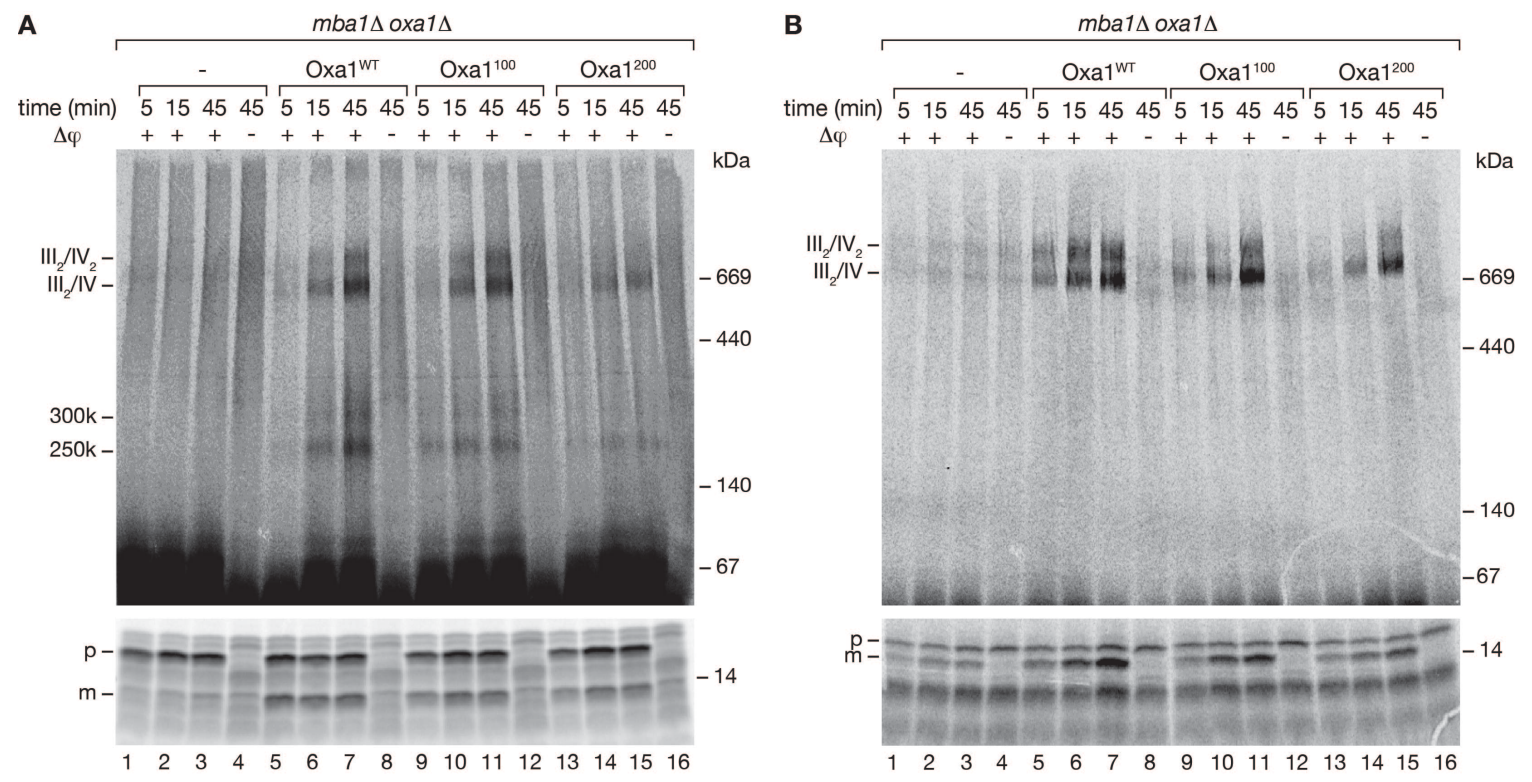

FIG 3.2 Import and assembly of radiolabeled Cox5a and Cox13 into 0xa1 linker mutants.

Import and assembly of (A) radiolabeled Cox5a (5 \%) and of (B) radiolabeled Cox13 (10\%). Lower panels: total import efficiency, analysed using SDS-PAGE. Upper panels: assembly of imported precursors into COX assembly intermediates ( 250 and $300 \mathrm{kDa}$ complexes, for Cox5a only) and supercomplexes $\left(\mathrm{III}_{2} \mathrm{IV}_{\mathrm{V}}\right.$ and $\mathrm{III}_{2} \mathrm{IV}_{2}$ ). precursor $(\mathrm{p})$, mature $(\mathrm{m})$. Import for indicated times in presence or absence of mitochondrial membrane potential $(+/-$ $\Delta \psi)$. Non imported precursor was digested with PK in all experiments. 
Import of the nuclear-encoded subunits Cox5a (FIG 3.2; A) and Cox13 (FIG 3.2; B) occurred at the same rate into strains expressing wild type or mutant forms of 0xa1, since a matured PK resistant product was detected in equal amounts (m, lower panels).

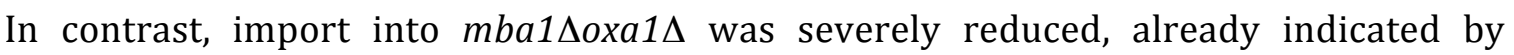
reduced steady state protein levels and the absence of supercomplexes in this strain (see FIG 3.1). In contrast to import rates, the assembly of both radiolabeled cytochrome $c$ oxidase subunits occurred with reduced kinetics in dependence of the linker length. In conclusion, insertion of a linker into the OXA1 sequence caused a cytochrome $c$ oxidase assembly defect, which was dependent on the length of the linker domain. This finding illustrated that spatial proximity of the mitochondrial ribosome to the site of membrane insertion is important for later steps of COX assembly.

These results were published in collaboration with the group of Prof. Herrmann, Kaiserslautern (Keil et al. 2012). 


\subsubsection{Isolation of new interaction partners of Mss51}

\subsubsection{C-terminally tagged Mss51 is fully functional}

Mss51 is not only a translational activator of Cox1 but acts also in early steps of cytochrome $c$ oxidase assembly (reviewed in Mick et al. 2011). In order to identify factors that support Mss51 in execution of these functions, we set out to isolate native protein complexes containing Mss51. To enrich cytochrome $c$ oxidase assembly intermediates at a very early stage, experiments were performed in a $\operatorname{coa} 1 \Delta$ yeast strain. Mss51 was tagged chromosomally with a Strep-FLAG tag. To test the functionality of Mss51-Strep-FLAG (Mss51 ${ }^{\mathrm{SF}}$ ), growth behavior of this strain was compared to a wild-type yeast strain (FIG 3.3; A). Both strains showed identical growth behavior on fermentable and non-fermentable media. In the coa1 $\Delta$ background, growth on non-fermentable media was absent as expected (Pierrel et al. 2007) but a tagged version of Mss51 did not alter growth behavior on YPD. Mitochondria from wild type and Mss51 ${ }^{\mathrm{SF}}$, grown on YPG, were prepared and steady state protein levels were analysed by SDS-PAGE and Western blotting (FIG 3.3; B).

A

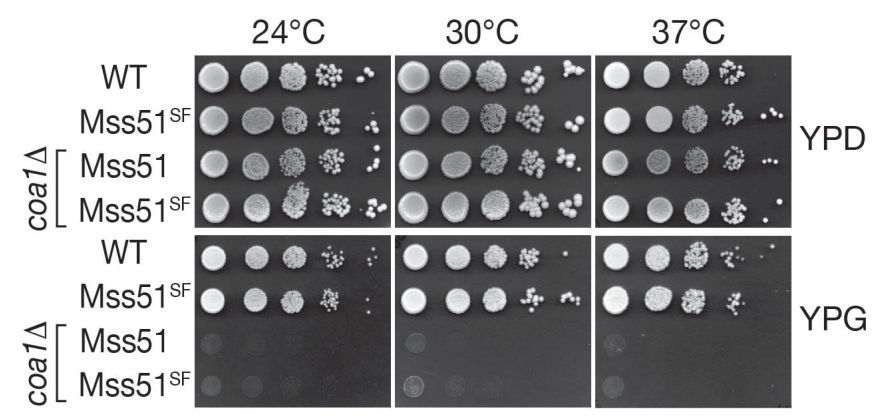

B

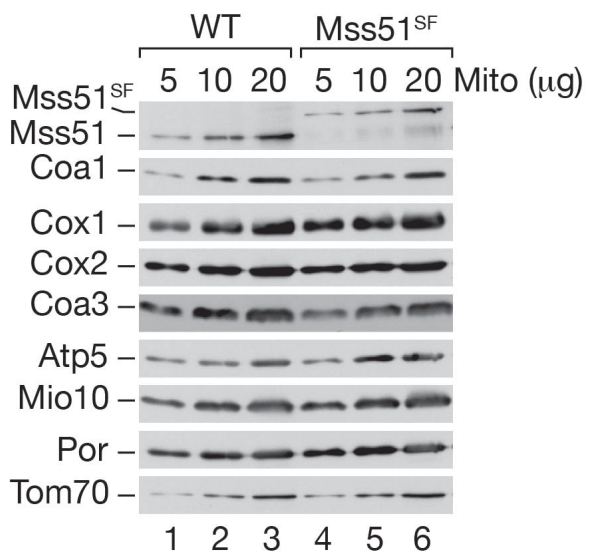

FIG 3.3 Analysis of growth behavior and steady state protein levels of strains with tagged Mss51

(A) Growth test on fermentable and non-fermentable full media. Cells were spotted in serial 10-fold dilutions and incubated at indicated temperatures. (B) Indicated amounts of isolated mitochondria (Mito) from different strains were separated by SDS-PAGE, followed by Western blot and probed for various mitochondrial proteins. 
To detect Mss51, an antibody directed against the whole protein was generated, thus allowing the detection of the wild type and tagged versions of Mss51. Mss51 ${ }^{\text {SF }}$ protein levels appeared reduced, compared to untagged Mss51. However, steady state levels of all other mitochondrial proteins tested were identical in both strains (FIG 3.3; B).

In summary, a C-terminal tag on Mss51 did not alter growth behavior nor steady state protein levels and we therefore concluded that it is fully functional and can be used for further experiments.

\subsubsection{SILAC-labeling efficiency}

To assess the incorporation of heavy arginine and lysine into yeast proteins, $\arg 4 \Delta \operatorname{coa} 1 \Delta$ cells, expressing Mss51 SF, were grown on SILAC media for five population doublings. Whole cell extracts were prepared and the incorporation efficiency (maximum value of each density function) was determined (FIG 3.4).

A

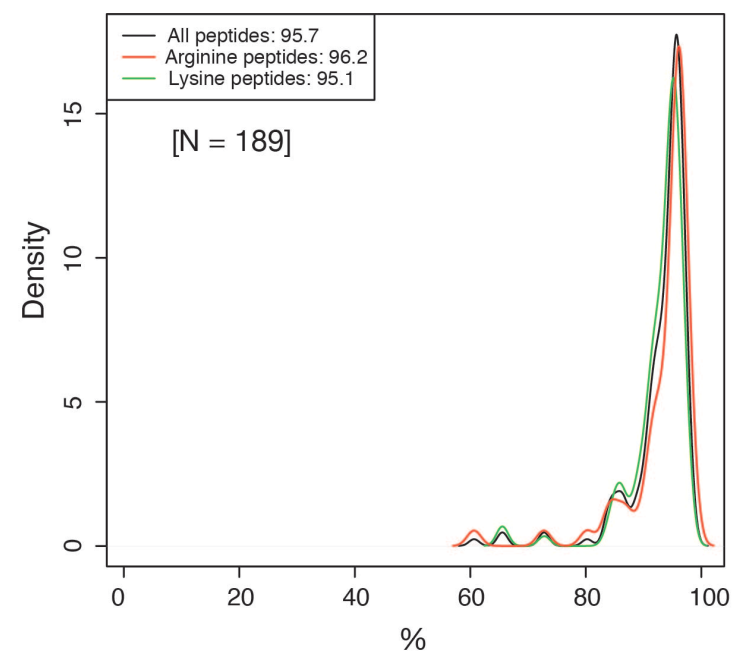

B

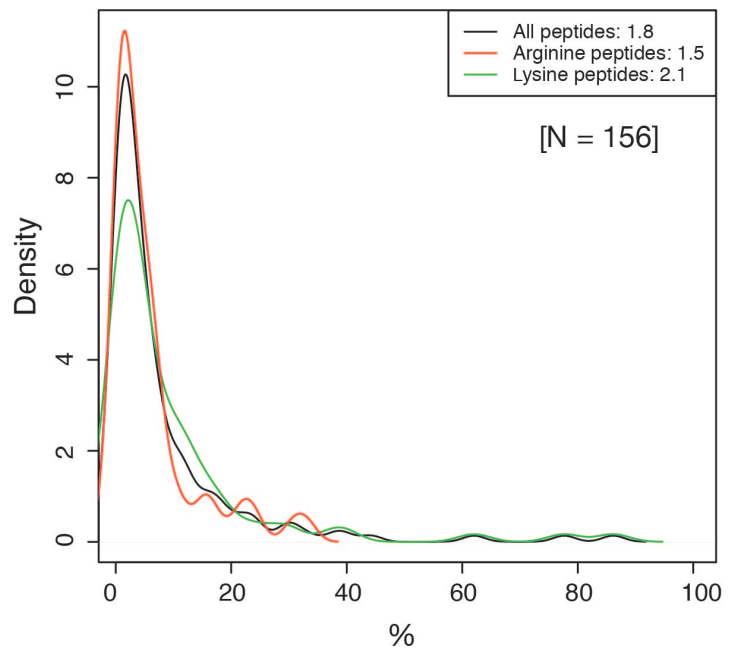

FIG 3.4 Efficiency of incorporation of heavy amino acids into yeast proteins

(A) Density function of SILAC ratios for heavy arginine (red) and lysine (green) containing peptides separately, and combined (black)). (B) Density function of SILAC ratios for light peptides, labeled as in (A). Total number of peptides used for the calculation (N). Figure prepared by M. Nikolov. 
The maximum value of each density function was used as incorporation efficiency and resulted in $96.2 \%$ for heavy arginine, $95.1 \%$ for heavy lysine and $95.7 \%$ for heavy arginine/lysine combined. At the same time, $1.5 \%$ of peptides still contained light arginine and $2.1 \%$ light lysine (1.8\% peptides with arginine/lysine combined). Taking into account the purity of the deployed amino acids ( $99 \%$ enrichment), almost complete incorporation of the supplemented heavy amino acids into the yeast proteins was achieved and stable isotope labeling with amino acids in yeast culture (SILAC) was successful.

\subsubsection{Interaction partners of Mss51 by SILAC}

Interaction partners found by mass spectrometry after native Mss51 SF isolation were Mss51 (bait protein), Ssc1, Shy1, Oms1, Cox5a, Cox6 and Cox1. All proteins were found to be enriched in the forward as well as in the reverse experiment using SILAC ratios $(\log 2 \mathrm{H} / \mathrm{L}$ and $\log 2 \mathrm{~L} / \mathrm{H})$ of above 2 in both experiments (FIG 3.5). One has to note that by isolating Mss51 complexes from coa1 $\Delta$, we accumulate one very prominent complex containing Mss51 at approximately $220 \mathrm{kDa}$ (FIG 3.6; B).

A

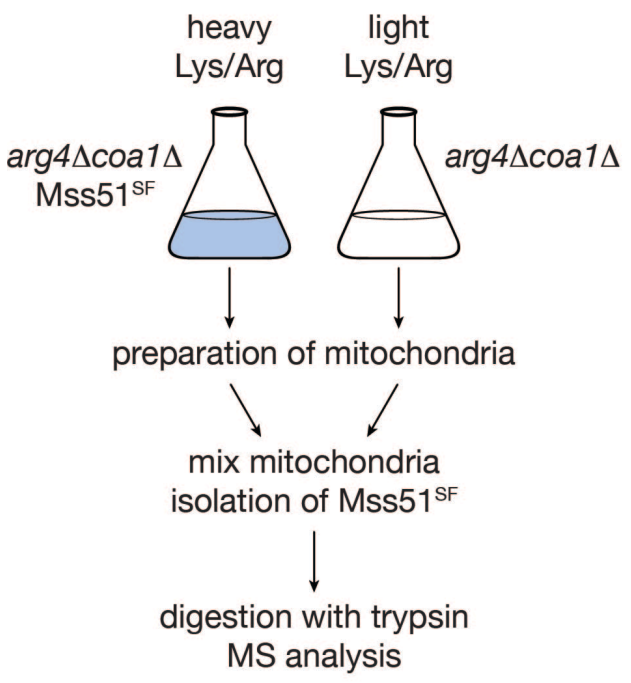

B

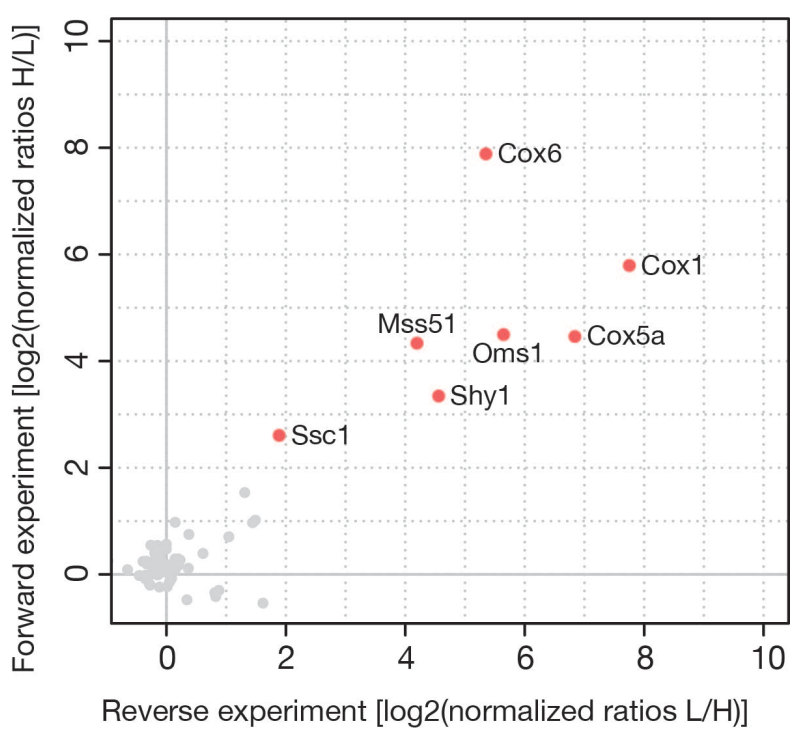

FIG 3.5 Stable isotope labeling with amino acids in cell culture (SILAC)

(A) Scheme of Mss51 isolation, combined with SILAC (Representation of forward experiment. For the reverse experiment, $\arg 4 \Delta \operatorname{coa} 1 \Delta \mathrm{Mss} 51^{\mathrm{SF}}$ was grown of light media and $\arg 4 \Delta \operatorname{coa} 1 \Delta$ on heavy media) (B) Scatter plot representation of normalized heavy/light (forward experiment) and light/heavy (reverse experiment) SILAC ratios after isolation of Mss51SF (via Strep-tag) from $\arg 4 \Delta \operatorname{coa} 1 \Delta$. Enriched proteins are displayed as red dots, other proteins in grey. 
Ssc1 was described before to interact with Mss51 and Mss51-containing complexes (Fontanesi et al. 2010). Copurification of Mss51 and Shy1 was also published by our laboratory (Mick et al. 2007; Mick et al. 2010). However we are the first to report an interaction of Oms1 with the translational activator Mss51. Oms1 was originally identified as a multicopy suppressor of respiratory defects, caused by OXA1 mutations (Lemaire et al. 2004). Isolation of Cox1 is in accordance with the current model of cytochrome $c$ oxidase assembly, however the isolation of Cox $5 \mathrm{a} / \mathrm{Cox} 6$ from cytochrome $c$ oxidase assembly intermediates in coa1s was surprising (compare FIG 1.6).

In-gel digestion, mass spectrometry, data analysis and visualization (also for the results presented in section 3.1.2.2) was performed in the Bioanalytical Mass spectromentry Group of Prof. Urlaub at the MPI BPC (Göttingen), mainly by Monika Raabe and Dr. Miroslav Nikolov.

\subsubsection{Confirmation of SILAC results by Western Blot analysis}

To confirm the results obtained by mass spectrometry analysis, proteins eluted under native conditions were also analysed by Western blotting after separation by SDS- or BN-PAGE (FIG 3.6; A and B). Isolation of Mss51 ${ }^{\mathrm{SF}}$ was successful in terms of efficiency and purity: except for a minor amount of Ssc1, no proteins were isolated from wild type mitochondria (control), indicating a high specificity of the isolation. Co-isolation of Cox1, Cox5a, Shy1 and Ssc1 was confirmed. Due to the lack of specific antibodies against Oms1, Western Blots could not be probed for the protein. Control proteins (translocase of the inner membrane component Tim50, soluble matrix protein Aco1, F $\mathrm{F}_{0}$-ATPsynthase component Atp5) were not found to precipitate with Mss51. In addition, Coa3 and Cox14 were found to specifically co-isolate with Mss51, a finding in accordance with previously published results (Barrientos et al. 2004; Pierrel et al. 2007; Mick et al. 2010; Fontanesi et al. 2010). 
A

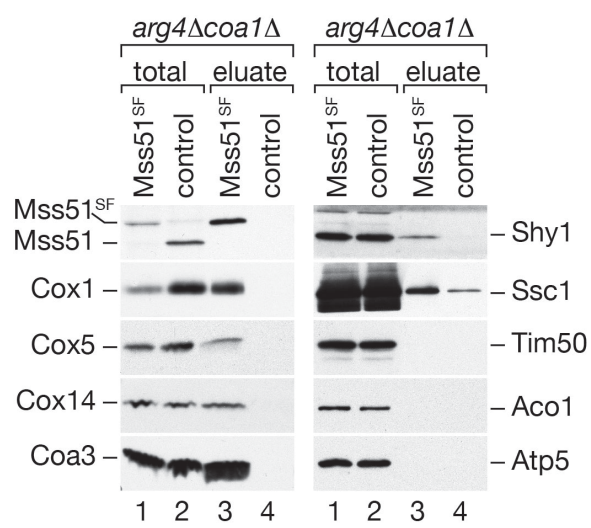

B

$\arg 4 \Delta \operatorname{coa} 1 \Delta$

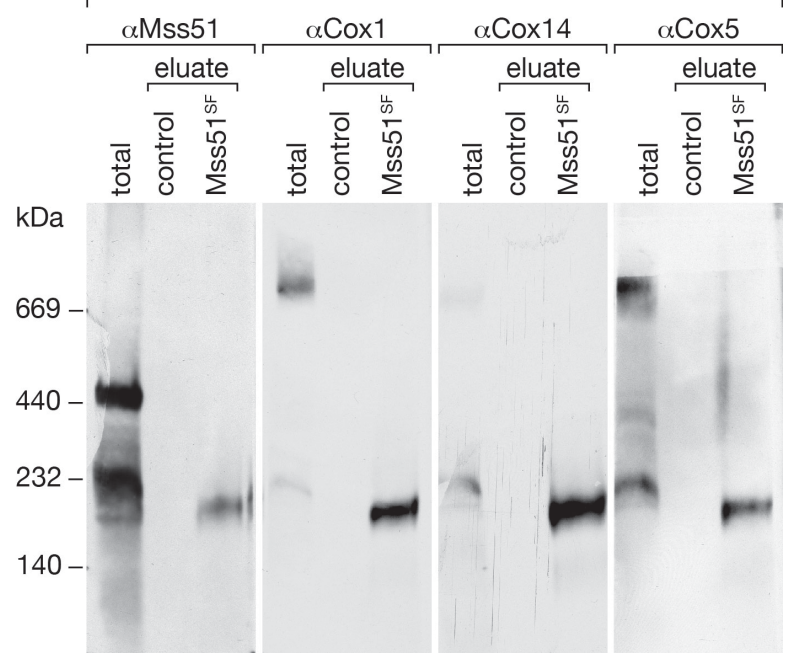

FIG 3.6 Analysis of eluates from Mss51 $1^{\text {SF }}$ purification by Western blotting

(A) Native purification of Mss51SF containing complexes by Strep-isolation. SDS-PAGE and Western blot analysis of total samples and eluates. Amount of total sample loaded corresponds to $3 \%$ of the eluate (B) Native eluted proteins (as in A) were analysed by BN-PAGE and Western blotting. As a control, purification was performed from solubilized wild type mitochondria.

Tryptic peptides of Coa3 and Cox14 were also found in the SILAC analysis (see section 3.1.2.3), however could not be illustrated in the scatter plot as the amount of peptides was not sufficient to determine a reliable heavy/light SILAC ratio. As Coa3 and Cox14 are both proteins of a very small molecular weight ( $9.8 \mathrm{kDa}$ or $7.9 \mathrm{kDa}$, respectively) with one hydrophobic domain that spans the inner mitochondrial membrane, insufficient tryptic digestion is speculated to account for the small amount of peptides obtained.

After separation of the eluates by BN-PAGE, the eluted proteins presented as one single entity at approximately $220 \mathrm{kDa}$. We could confirm the presence of Mss51, Cox1, Cox14 and Cox5 by Western blotting. 


\subsubsection{Nuclear-encoded subunits Cox5a and Cox6 in early COX assembly}

\subsubsection{Import and assembly of radiolabeled Cox5a into COX assembly mutants}

Using steady state analysis of respiratory chain complexes in mitochondria from different COX assembly mutants, we found an assembly intermediate of cytochrome $c$ oxidase accumulating in coa $1 \Delta$, but also in shy1 $\Delta$ cells (FIG 3.7, A). This COA complex is of approximately $220 \mathrm{kDa}$ in size, identical to the complex isolated via Mss51 ${ }^{\mathrm{SF}}$ from coa1s. In shy1s, this intermediate was slightly shifted towards a higher molecular weight, giving a hint towards the presence of Coa 1 in this complex. In coa $3 \Delta$ and $\operatorname{cox} 14 \Delta$, no complexes could be detected due to the absence of Cox1, which is in accordance with previous findings (Merz \& Westermann 2009; Mick et al. 2010). Decoration against $\mathrm{F}_{1} \mathrm{~F}_{0}$-ATP-synthase subunit Atp5 was used to ensure equal loading. To confirm the presence of Cox5a in cytochrome $c$ oxidase assembly intermediates accumulating in coa1s and shy $1 \Delta$, we imported radiolabeled Cox5a and followed its assembly into complexes (FIG 3.7; B).
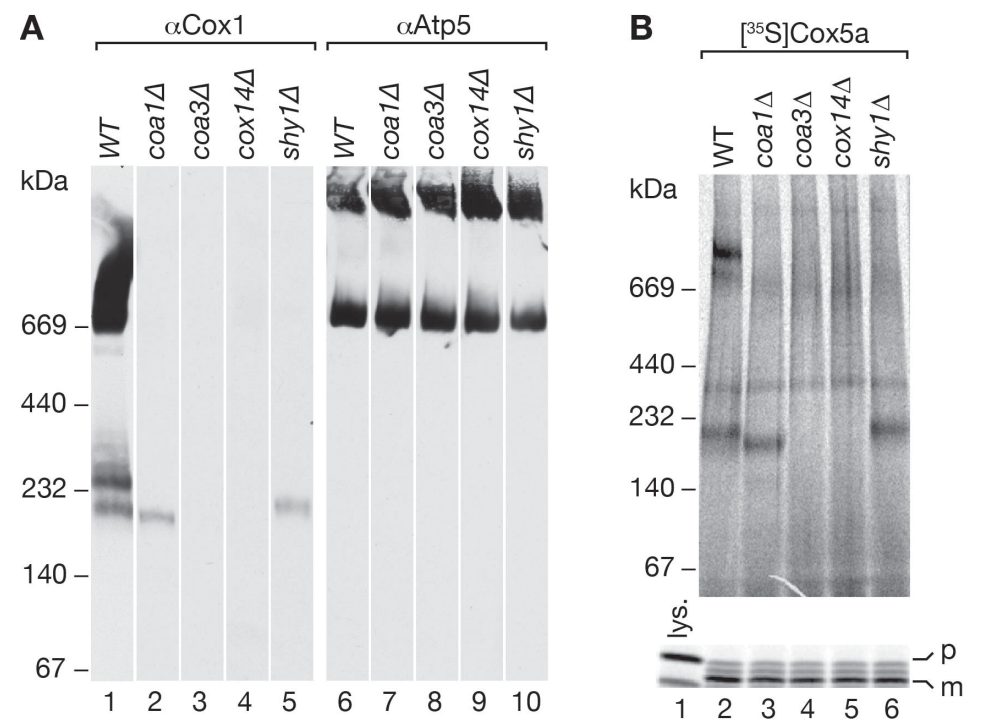

FIG 3.7 Analysis of accumulated COA complexes for presence of Cox5a

(A) $50 \mu \mathrm{g}$ mitochondria, isolated from indicated strains were separated by 4-13\% BN-PAGE, followed by Western blot analysis and probing for Cox1 and Atp5. (B) Import and assembly of radiolabeled Cox5a into isolated mitochondria from indicated strains. 5\% lysate. Lower panel: total import efficiency, by SDS-PAGE. Upper panel: assembly of imported precursors into COX assembly intermediates and supercomplexes (III $I_{2} \mathrm{IV}$ and $\mathrm{III}_{2} \mathrm{IV}_{2}$ ), resolved by $4-13 \%$ BN-PAGE. Import for $45 \mathrm{~min}$ in presence of mitochondrial membrane potential. Unimported precursor was digested with PK. precursor (p), mature (m). 
Import occurred with identical efficiency into mitochondria from all strains, as judged by the amount of matured and PK-protected Cox5a (FIG 3.7; B, lower panel). In wild type mitochondria, Cox5a assembled into one prominent COA complex of around $220 \mathrm{kDa}$ in size and into the two supercomplexes, containing cytochrome $c$ oxidase $\left(\mathrm{III}_{2} \mathrm{~V}\right.$ and $\left.\mathrm{III}_{2} \mathrm{~V}_{2}\right)$. In coa1 $\Delta$, Cox $5 \mathrm{a}$ assembled only into the COA complex, due to a lack of mature oxidase already observed on steady state levels (FIG 3.7; A) and published previously (Pierrel et al. 2007; Mick et al. 2007). The COA complex, observed in coa1 migrated slightly faster compared to wild type indicating a lower molecular weight. As mentioned above, we speculated that this size shift was due to the absence of the Coa1 protein. In shy $1 \Delta$, we also observed an accumulating complex, containing radiolabeled Cox5a of approximately $220 \mathrm{kDa}$.

In conclusion, we showed the presence of Cox $5 \mathrm{a}$ in the $220 \mathrm{kDa}$ complex, accumulating in coa1 $\Delta$. This finding confirmed the results from SILAC analysis. Furthermore, we speculated that in shy1 $\Delta$ cells, a similar COA complex is accumulating, which differs only in the presence or absence of the Coa1 protein. 


\subsubsection{Requirement of Cox6 for recruitment of Shy1 to COA complexes}

The presented data raised the question if Cox $5 \mathrm{a}$ is required for the recruitment of Shy 1 to the COA complexes. As Cox5 is present in two isoforms (Cox5a and Cox5b), we made use of the fact that Cox 6 is needed to stabilize both isoforms of Cox 5 by protecting them from proteolytic instability. In cox $6 \Delta$, only trace amounts of Cox $5 a$ or Cox $5 b$ were detectable (Glerum \& Tzagoloff 1997; Church et al. 2005). Therefore, we performed the following studies in mitochondria isolated from $\operatorname{cox} 6 \Delta$, grown on YPGal. To test whether Cox6 is required to recruit Shy1 to COA complexes, we followed two independent approaches.

First, we isolated native complexes, containing Shy1-ProteinA (Shy1 ${ }^{\text {ProtA }}$ ) using IgGchromatography from digitonin solubilized mitochondria (FIG 3.8, A). This experiment was performed in a wild type (FIG 3.8; A, lanes 5 and 6) as well as in a $\operatorname{cox} 6 \Delta$ background (FIG 3.8; A, lanes 3 and 4). As a control, IgG-chromatography was also performed from cox6 6 , without a tagged Shy1 version present (FIG 3.8, A; lanes 1 and 2). In total samples of $\operatorname{cox} 6 \Delta$, we observed no Cox6 and only trace amounts of Cox5, Cox1 or Cox2. Steady state levels of other proteins were comparable to wild type levels. Isolation efficiency of Shy1 $1^{\text {ProtA }}$ was identical in wild type and $\operatorname{cox} 6 \Delta$ mitochondria. In wild type mitochondria, all known components of COA complexes (Coa3, Cox1, Cox5, Cox6, Mss51, Coa1 and Cox14) were co-isolated with Shy1 ${ }^{\text {ProtA. }}$

A

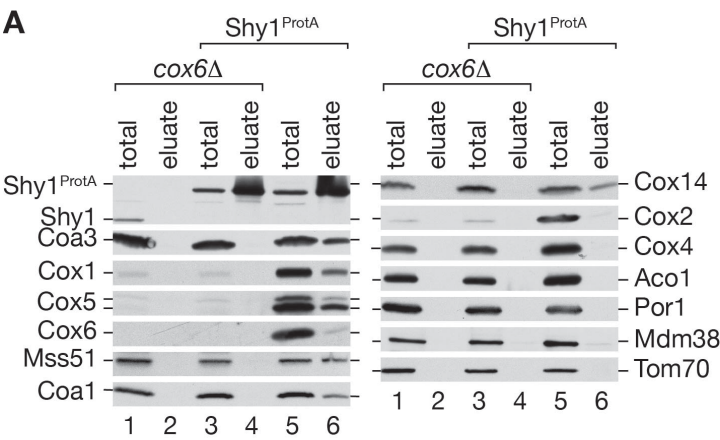

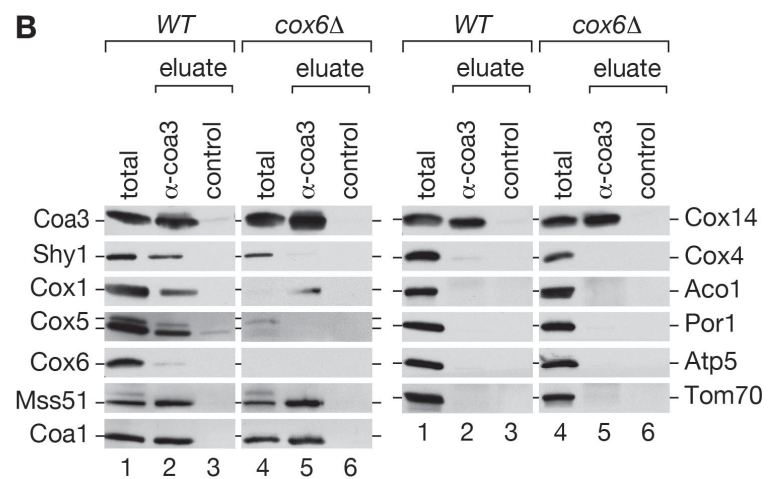

FIG 3.8 Cox6 is required for recruitment of Shy1 to COAs

(A) Mitochondria from $\operatorname{cox} 6 \Delta$ and TEV-ProteinA-7His tagged Shy1 (Shy1 ${ }^{\text {ProtA }}$ ) in wild type or cox $6 \Delta$ background were solubilized in $1 \%$ digitonin buffer and subjected to IgG-chromatography. After acidic elution, the eluate was separated on SDS-PAGE and analysed by Western blotting, (total 1.5\%, eluate 100\%) (B) Coimmunoprecipitation of Coa3 (and control) from digitonin-solubilized mitochondria, isolated from wild type (WT) and cox6 6 were analysed by SDS-PAGE and Western blotting (total 8\%, eluate 100\%). 
In contrast, in $\operatorname{cox} 6 \Delta$, none of the mentioned cytochrome $c$ oxidase assembly factors or early assembling subunits were found to co-isolate with Shy1 ${ }^{\text {ProtA }}$. In both cases, none of the tested control proteins (Cox2, $\operatorname{Cox} 4$, Aco1, Por1, Mdm38, Tom70) were copurified. Thus, we concluded that Cox6 is required for the association or recruitment of Shy 1 to COA complexes.

In the second approach, we performed co-immunoprecipitation against Coa3 to isolate COA complexes (FIG 3.8, B). This experiment was performed with solubilized mitochondria, isolated from wild type yeast cells (FIG 3.8; B, lanes 1-3), compared to mitochondria derived from cox6s cells (FIG 3.8, B; lanes 4-6). As a control, coimmunoprecipitation was performed using antiserum against Yhl47. Antibodies directed against Coa3 efficiently precipitated Coa3 both from wild type and $\operatorname{cox} 6 \Delta$ mitochondria. With Cox14, Shy1, Cox1, Cox5, Mss51 and Coa1, COA components were recovered from wild type mitochondria, in accordance with previously published results (Mick et al. 2010; Reinhold et al. 2011; Fontanesi et al. 2011). In addition, minor amounts of Cox6 were copurified. Other subunits of cytochrome $c$ oxidase that assemble later to the maturing enzyme ( $\operatorname{Cox} 2, \operatorname{Cox} 4)$ were not identified in the eluates. All control proteins tested (Aco1, Por1, Atp5 or Tom70) were not found to co-isolate, underlining specificity of the detected proteins. However, we noticed differences between the isolation from wild type and $\operatorname{cox} 6 \Delta$ : Shy1 was not found in the eluate, despite comparable levels of the protein in total samples of wild type and $\operatorname{cox} 6 \Delta$. Furthermore, Cox5 and Cox6 could not be detected in the eluate from $\operatorname{cox} 6 \Delta$, a finding that is explained by the absence of Cox 6 and the minor residual amount of Cox 5 present at steady state levels in $\operatorname{cox} 6 \Delta$ mitochondria. Despite the small amount of $\operatorname{Cox} 1$ in $\operatorname{cox} 6 \Delta$, the remaining protein was present in Coa3-containing complexes, indicating the accumulation of Cox1-containing assembly intermediates in $\operatorname{cox} 6 \Delta$. Taken together, we present additional evidence that in the absence of Cox 5 and Cox6, Shy 1 was not present in COA complexes.

In conclusion, we could show the requirement of Cox6 (and of Cox5 indirectly) for the recruitment of Shy1 to COA complexes by two independent methods. 


\subsubsection{Studying defects in COX assembly in Leigh syndrome: Characterization of a SHY1 point mutation (transferred from patient SURF1 mutation)}

\subsubsection{Growth behaviour of Shy $1^{\mathrm{YD}}$ is affected}

Cytochrome $c$ oxidase deficiency is one of the major causes of Leigh syndrome, a fatal encephalopathy of infancy. Mutations in SURF1 are the most prevalent cause of Leigh syndrome with isolated COX deficiency (Péquignot et al. 2001). In patients, various mutations were found, amongst others a point mutation leading to an exchange of a conserved tyrosine at position 274. We used the yeast homolog of SURF1, Shy1 as a

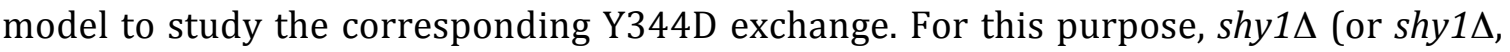
expressing Mss51 ${ }^{\mathrm{HA}}$ for later detection purposes) cells, carrying an empty plasmid or expressing the wild type or mutant form of Shy1 were generated. Strains expressing the mutant form of Shy1 from a plasmid under its endogenous promotor showed a cold sensitive respiratory deficiency with slightly reduced growth on non-fermentable carbon source at $30^{\circ} \mathrm{C}$ (FIG 3.9; A and Reinhold et al. 2011).

A

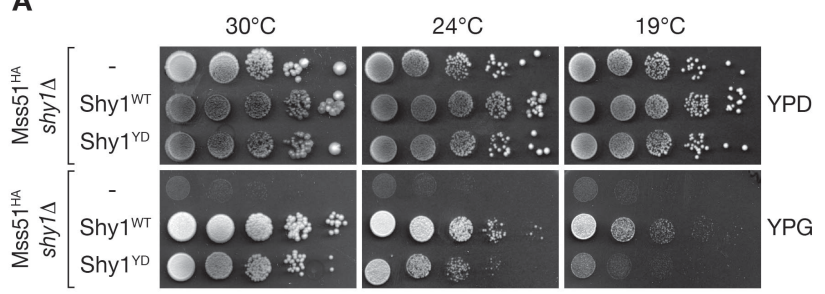

B

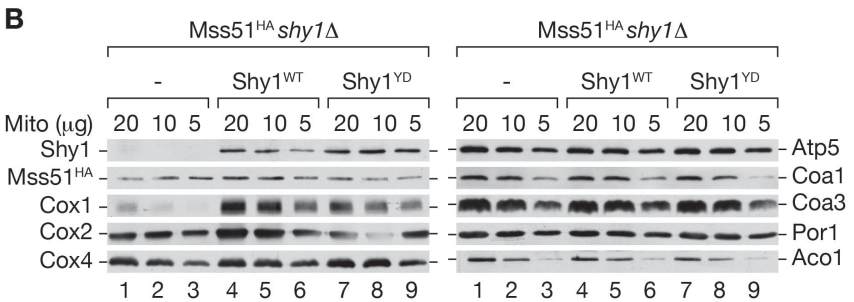

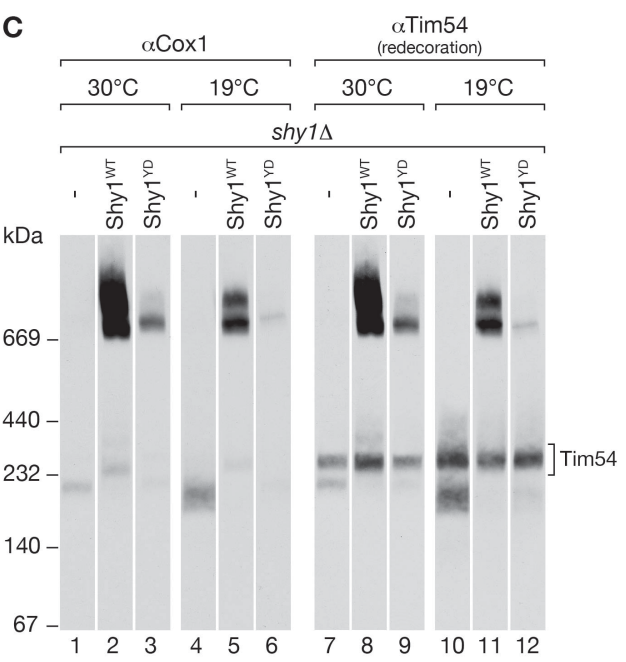

FIG 3.9 Analysis of growth behavior and steady state protein levels of strains with a Shy1 point mutation (Shy1 ${ }^{\text {YD) }}$

(A) Serial dilutions of Mss51 ${ }^{\mathrm{HA}}$ shy $1 \Delta$ cells, containing an empty plasmid (-) or expressing Shy 1 WT or Shy $1^{\mathrm{YD}}$ were spotted onto fermentable (YPD) or non fermentable (YPG) solid medium and incubated at indicated temperatures. (B) Indicated amounts of mitochondria, isolated from strains described in (A), grown at $30{ }^{\circ} \mathrm{C}$ in SGal-Ura, were analysed by SDS-PAGE and Western blotting using indicated antibodies against COX subunits and assembly factors as well as control proteins. (C) $30 \mu \mathrm{g}$ mitochondria, isolated from shy1 $\Delta$ cells, containing an empty plasmid (-) or expressing Shy1 ${ }^{\mathrm{WT}}$ or Shy $1^{\mathrm{YD}}$, grown in SGal-Ura at 30 or $19^{\circ} \mathrm{C}$. Separation by $4-13 \% \mathrm{BN}-$ PAGE, followed by Western blotting against Cox1, redecoration with Tim54 
To assess this phenotype in a more direct way, cells from described strains were grown in SGal-Ura at $30^{\circ} \mathrm{C}$ and steady state proteins levels were analysed by SDS-PAGE (FIG 3.9; B and Reinhold et al. 2011). At $30^{\circ} \mathrm{C}$, we observed reduced levels of mitochondriaencoded cytochrome $c$ oxidase core components, such as Cox1 and Cox2 in Shy $1^{\mathrm{YD}}$ mitochondria, compared to wild type mitochondria (Bestwick, Jeong, et al. 2010a; Reinhold et al. 2011). Control proteins of the different mitochondrial subcompartments, as well as a number of early assembly factors such as Coa1, Coa3 and Mss51, were unaltered in the tested mitochondria.

To analyse the cold sensitive phenotype in more detail, mitochondria were prepared from cells grown on SGal-Ura at permissive $\left(30^{\circ} \mathrm{C}\right)$ and non-permissive $\left(19^{\circ} \mathrm{C}\right)$ temperatures. Subsequently, steady state levels of cytochrome $c$ oxidase, using antibodies against Cox1 were analysed (FIG 3.9; C and Reinhold et al. 2011). As reported previously, in shy1s, no mature cytochrome $c$ oxidase was detected (Mashkevich et al. 1997; Nijtmans et al. 2001). In Shy1 ${ }^{\mathrm{YD}}$, only a reduced amount of cytochrome $c$ oxidase could be detected, confirming the results of the SDS-PAGE analysis (FIG 3.9, B). Consistent with the temperature sensitive growth, cytochrome $c$ oxidase levels in Shy $1^{\mathrm{YD}}$, grown at $19^{\circ} \mathrm{C}$ were hardly detectable. Redecoration with antibodies against Tim54 was used to ensure equal loading (complex of $300 \mathrm{kDa}$, in Shy $1^{\text {WT }}$ overlapping with the signal of Cox1 monomer). Taken together, we concluded that the respiratory deficiency observed in Shy $1^{\mathrm{YD}}$ was caused by a reduction of cytochrome $c$ oxidase; especially of its mitochondria-encoded subunits.

In Reinhold et al. (2011), we reported that import efficiency and stability of Shy $1^{\mathrm{YD}}$ was not affected, compared to wild type Shy1 (the same was true for SURF1 ${ }^{\mathrm{Y} 274 \mathrm{D}}$, which accumulated in a cytochrome $c$ oxidase assembly intermediate). Furthermore, the topology of Shy $1^{\mathrm{YD}}$ in the membrane was not altered. However, Shy $1^{\mathrm{YD}}$ uncouples Cox1 expression from COX assembly: even though COX assembly was disturbed in Shy $1^{\mathrm{YD}}$ cells, Cox1 translation rate was found to be comparable to wild type. Using pulse-chase experiments, we could show that newly synthesized Cox1 was very instable, resulting in reduced steady state levels of Cox1. (Generation of the mutant form of Shy1, import and stability assays, membrane topology determination, in vivo labeling of translational products and chase experiments were performed by Dr. R. Reinhold in the Department of Cellular Biochemistry, Göttingen). 


\subsubsection{Accumulation of COA complexes in Shy1(YD)FLAG mitochondria}

In cells expressing Shy $1^{\mathrm{YD}}$, Cox1 translation was not shut down although cytochrome $c$ oxidase assembly was defective. By Coa3 and Coa1 co-immunoprecipitation, we could not show accumulation of COA complexes, judged from SDS-PAGE (Reinhold et al. 2011). Hence, we concluded that Mss51 was not sequestered by Shy $1^{\text {YD }}$ but was rather active and able to constantly trigger translation of COX1 mRNA. However, due to the denaturing (acidic) elution after co-immunoprecipitation, eluates could not be analysed by BN-PAGE.

In order to investigate the reason for the normal Cox1 synthesis rate in more detail, we set out to isolate the mutant and wild type Shy1 and subsequently identify its interaction partners in native complexes by BN-PAGE. For this purpose, we generated plasmids, containing FLAG-tagged versions of Shy $1^{\text {WT }}$ or Shy $1^{\text {YD }}$ (plasmids were generated by Dr. D. Mick in the Department of Cellular Biochemistry, Göttingen). These plasmids (and an empty vector control) were transformed into shy1 $\Delta$ cells, expressing Mss51 ${ }^{\mathrm{HA}}$. The Mss51 ${ }^{\mathrm{HA}}$ background was chosen for detection purposes as an antibody, recognizing native Mss51, was not available at that time. After FLAG-isolation under native conditions, we analysed the eluate using SDS-PAGE and Western blotting (FIG $3.10 ; \mathrm{A})$.
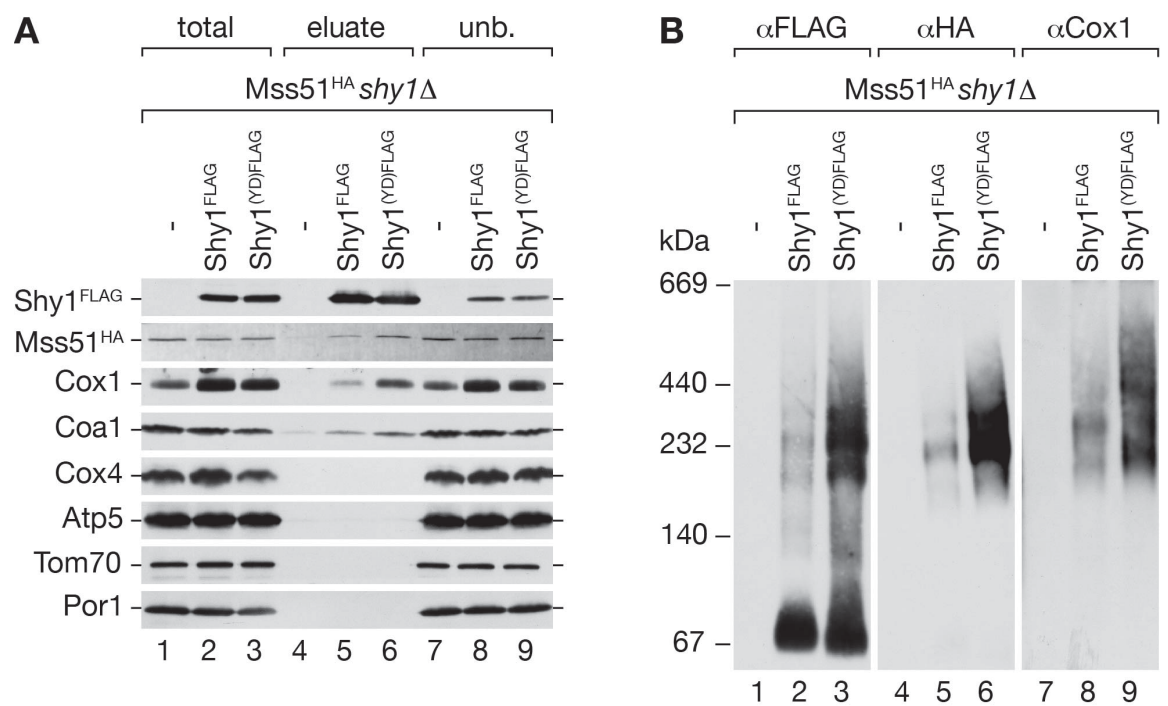

FIG 3.10 Native isolation of Shy1 FLAG / Shy1(YD)FLAG

(A) Western blot analysis of elution of FLAG-isolation from digitonin lysed mitochondria from indicated strains. Native elution via FLAG peptide. Amount of protein loaded in the total and unbound (=unb.) samples correspond to $9 \%$ of the elution. (B) The native elution from (A) was analysed by $4-13 \%$ BN-PAGE, followed by Western blotting. Decoration against FLAG and HA with monoclonal antibodies, decoration for Cox1 with polyclonal antibodies. 
The isolation efficiency of Shy $1^{\mathrm{YD}}$ was comparable to the wild type version. However, the amount of Cox1, isolated with the mutant form was significantly higher (compare FIG 3.10; B, lane 6 to lane 5) than in wild type, which is, to a lesser extent, also true for the amount of co-isolated Mss51. Subsequently, to resolve these differences, the eluates were separated on a native gel. Shy $1^{\text {WT }}$ was found in COA complexes (identified by the presence of Cox1, Mss51 and Shy1) but predominantly in a $70 \mathrm{kDa}$ complex. To our surprise, Shy $1^{\mathrm{YD}}$ was in contrast mainly present in accumulated COA complexes.

In contrast to our previous work (Reinhold et al. 2011), we found the accumulation of COA complexes containing Shy1 and Mss51. This intermediate could not be detected at steady state levels (Fig 3.9; C) but becomes visible after enrichment via FLAG isolation. We speculated that Mss51 is sequestered in this complex but the mutant form of Shy1 was not able to transform it into its inactive form, hence Mss51 was still able to initiate further rounds of Cox1 synthesis. 


\subsection{Mapping Shy1-containing COA complexes: Association of heme a synthase with Shy1}

\subsubsection{Isolation of interaction partners of Shy $1^{\text {ProtA }}$}

Shy1 is required for Cox1 biogenesis and hence is part of early assembly intermediates in yeast, termed COA (Mick et al. 2007; Pierrel et al. 2007; Fontanesi et al. 2011). Furthermore, Shy1 plays a central role in early assembly steps of Cox1 during cytochrome $c$ oxidase maturation. Previous analyses found Shy1 in several distinct mitochondrial protein complexes, most of which have been resolved by BN-PAGE analyses (Nijtmans et al. 2001; Mick et al. 2007; Mick et al. 2010; Fontanesi et al. 2011). However, a comprehensive analysis of the protein composition of these complexes was still lacking.

To assess the composition of these intermediates, we isolated assembly intermediate complexes in their native state and analysed the components utilizing a combination of Western blotting and mass spectrometry. For this purpose, we used a functional fusion construct of Shy1 (Mick et al. 2007). Shy1 was chromosomally tagged with a protein A tag at the C-terminus (Shy $1^{\text {ProtA })}$ that can be cleaved by TEV (tobacco etch virus) protease treatment to release native Shy1-containing complexes. Shy1 ${ }^{\text {ProtA }}$ was purified by IgG-chromatography from isolated wild type or Shy $1^{\text {ProtA }}$ mitochondria after mild solubilization in digitonin-containing buffer. Subsequently, Shy1 and its associated proteins were cleaved from the resin by TEV protease treatment and analysed either by SDS-PAGE or BN-PAGE, followed by Western blot or mass spectrometric analysis (FIG $3.11 ; \mathrm{A})$
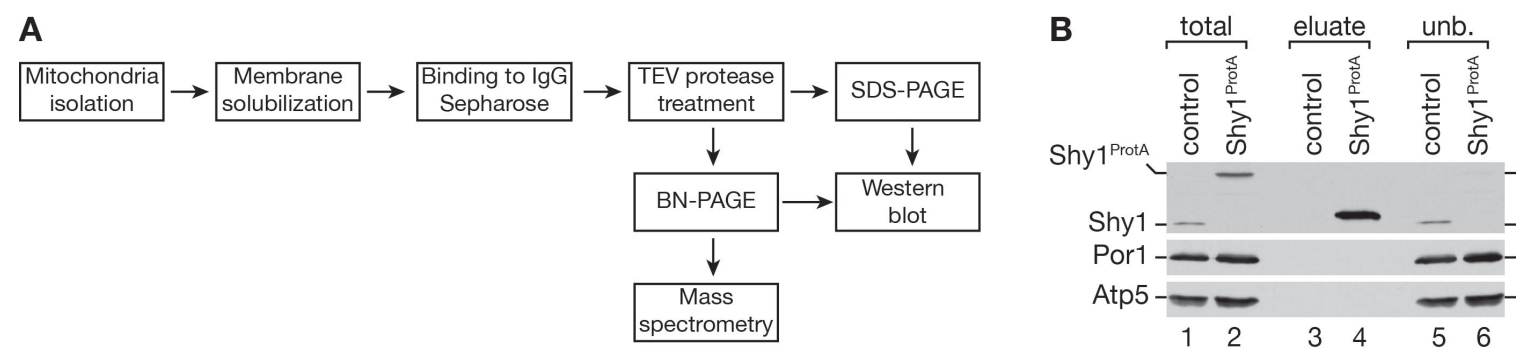

FIG 3.11 Isolation of Shy1ProtA via IgG chromatography

(A) Schematic illustration of the native purification of Shy1 Prota by IgG-chromatography and different analytical methods used. (B) Total samples, TEV-cleaved elution and supernatant (=unb.) of IgG-chromatography from mitochondria, isolated from wild type and Shy1ProtA were analysed by SDS-PAGE, followed by Western blotting. Amounts of protein loaded in the total samples correspond to $5.5 \%$ of the elution. 
This approach allowed efficient purification of Shy1 from mitochondrial extracts, which we assessed by SDS-PAGE and Western blotting of the different fractions (FIG 3.11; B) since complete depletion of Shy1 from the extract was achieved. After TEV cleavage, Shy1 migrates at lower molecular weight in an SDS gel but due to a short linker between the protein and the TEV site, still runs slightly different from wild type Shy1. No unspecific binding was observed as judged by the absence of control proteins (Atp5 and Por1) in the eluate fraction.

Based on the successful small scale isolation, we performed IgG-chromatography in a preparative way. The native elution was split in two unequal amounts and both seperated by BN-PAGE. The smaller fraction of the elution was analysed by Western blot and revealed several distinct complexes, containing Shy1 in the range of 80$440 \mathrm{kDa}$ (FIG 3.12; A).
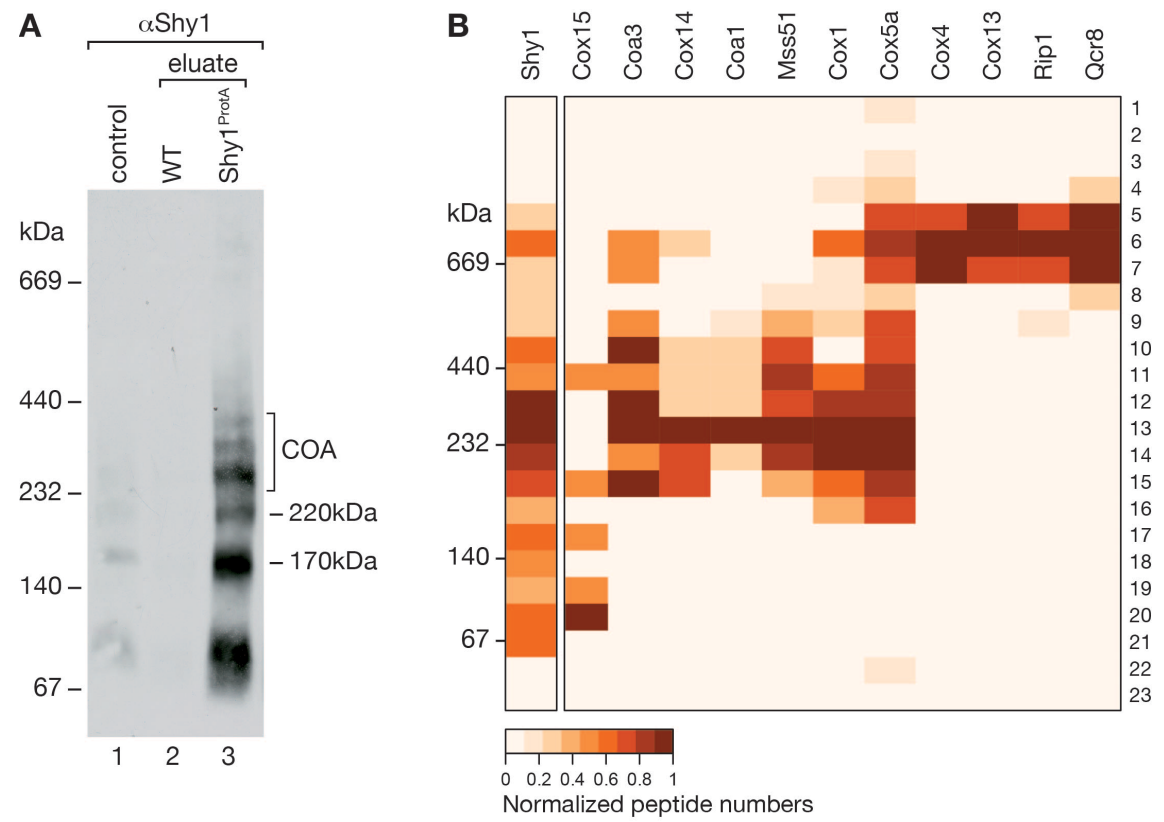

FIG 3.12 Mapping of Shy1 containing complexes by mass spectrometry

(A) A fraction of native eluted proteins of a preparative isolation of Shy1 ProtA, analysed by 4-13\% BN-PAGE and Western blotting for Shy1ProtA-containing complexes. As a control, solubilized wild-type mitochondria were loaded (6.5\% of eluate). (B) Majority of eluted proteins from (A) was separated by $4-13 \%$ BN-PAGE, followed by mass spectrometry. Heat map illustrating normalized peptide numbers of proteins co-isolated with Shy $1^{\text {ProtA }}$ in individual gel slices. Positions of marker bands were estimated from their running distances in a Coomassiestained gel lane. In-gel digestion, mass spectrometry, data analysis and visualization was performed in the Bioanalytical Mass spectrometry Group of Prof. Urlaub at the MPI BPC (Göttingen), mainly by Monika Raabe and Dr. Miroslav Nikolov. 
As a control, total mitochondrial extract was loaded. In accordance with results from SDS-PAGE, the elution from IgG sepharose, incubated with solubilized wild type mitochondria, contained no Shy1 complexes. To define the protein composition of Shy1 containing complexes in an unbiased manner, the majority of the elution, separated by BN-PAGE, was analysed by mass spectrometry. The gel lane was cut into 23 pieces which were independently subjected to in-gel trypsin digestion, followed by mass spectrometry (for detailed results, see Table S1 in supplemental material, Bareth et al. 2013). A heatmap, illustrating normalized peptide numbers of selected proteins in individual gel slices revealed the distribution of several Shy1-containing complexes (FIG 3.12; B). Cytochrome $c$ oxidase assembly factors, such as Coa3, Cox14, Coa1 and Mss51, were found in COA complexes, together with Shy1, migrating at 270-350 kDa. Cox1 and Cox5a were predominantly present in COA complexes but were also found in the high molecular weight range. It has been previously published that a fraction of respiratory chain supercomplexes are co-isolated with Shy1 (Mick et al. 2007; Vukotic et al. 2012), a fact that is not fully understood but is supported by the presence of components of $b c_{1}$ complex (Rip1, Qcr8).

Interestingly, we observed distinct complexes of the heme $a$ synthase Cox15 with Shy1. This finding agreed with our previous observation that Cox15 could be identified by mass spectrometry in Shy1 complexes purified from digitonin-solubilized membranes (Mick et al. 2007). However, to our surprise, Cox15 was not only found in low molecular complexes with Shy1 but was also associated with COA complexes. To confirm these unexpected result, we performed an independent experiment and analysed the Shy1containing complexes, separated by BN-PAGE with available antibodies (FIG 3.13). In agreement with mass spectrometric data, Shy1, Mss51, Coa3 and Cox14, together with the structural subunits Cox 1 and Cox5a, were detected in COA complexes but not in the smaller complexes. This finding suggested novel complexes, composed of Shy1 and Cox15 that lack known assembly factors or structural subunits of COX. In order to detect Cox15, we generated a polyclonal antiserum in rabbit, directed against an epitope at the C-terminus of the Cox15 protein. We detected Cox15 in complexes that were isolated with Shy $1^{\text {ProtA }}$ on BN-PAGE. Although the majority of Cox15 was found in the faster migrating complexes that lack COA components or structural COX subunits, a minor fraction of Cox15 co-migrated with COA complexes (FIG 3.13). 


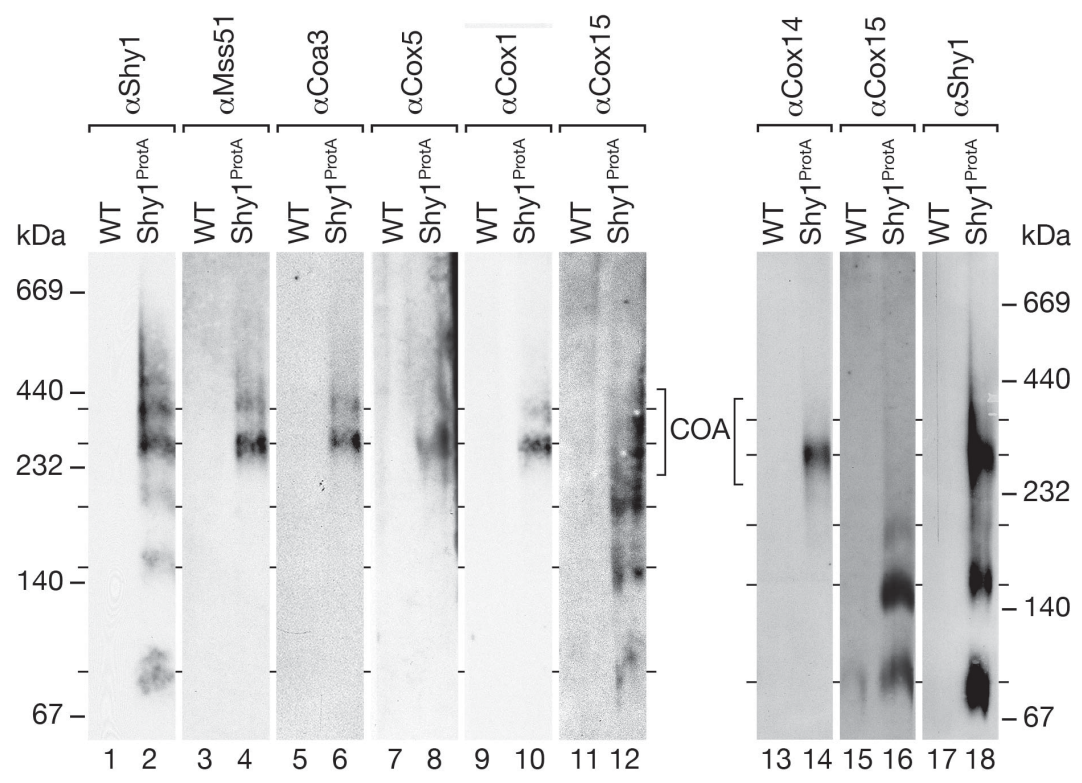

FIG 3.13 Mapping of Shy1 containing complexes by Western blotting

Western blot analysis of protein complexes after IgG chromatography from isolated wild type (WT) and Shy 1 ProtA mitochondria, separated by $4-13 \%$ BN-PAGE. Two independent experiments are shown.

The heme $a$ synthase Cox15 is required for the hydroxylation of heme $o$ to form heme $a$, which is an essential prosthetic group for cytochrome $c$ oxidase (Barros et al. 2001). Two heme $a$ molecules generated by this pathway are incorporated into the Cox 1 subunit of COX, however this process is not understood. A study in the bacterium P. denitrificans indicated that Surf1 (a homolog of yeast Shy1) is capable of binding heme $a$ in vitro, thus linking its function to the heme incorporation step for Cox1 during cytochrome $c$ oxidase biogenesis (Bundschuh et al. 2009).

The presented data allowed us to speculate that the transfer of heme $a$ into Cox 1 is achieved by a cooperated action of Shy1 and Cox15. 


\subsubsection{A C-terminal tag on Cox15 affects its association with COA complexes}

\subsubsection{Growth behaviour of strains with C-terminally tagged Cox15}

In order to follow the association of Cox15 with COA complexes, we integrated a Protein A or FLAG tag-encoding cassette into the chromosomal COX15 locus. This allows the purification of native Cox15-containing complexes. To test whether the tagged versions of Cox15 were functional, we analysed the growth of the strains on fermentable and non-fermentable carbon sources at different temperatures (FIG 3.14). Growth of the tested strains was identical to wild type on both carbon sources and at all temperatures tested. As a control, the respiratory deficient strain coa $3 \Delta$ was used (Merz \& Westermann 2009; Mick et al. 2010; Fontanesi et al. 2011). Hence we concluded that C-terminal tagged Cox $15^{\text {ProtA }}$ and Cox $15^{\mathrm{FLAG}}$ were functional.

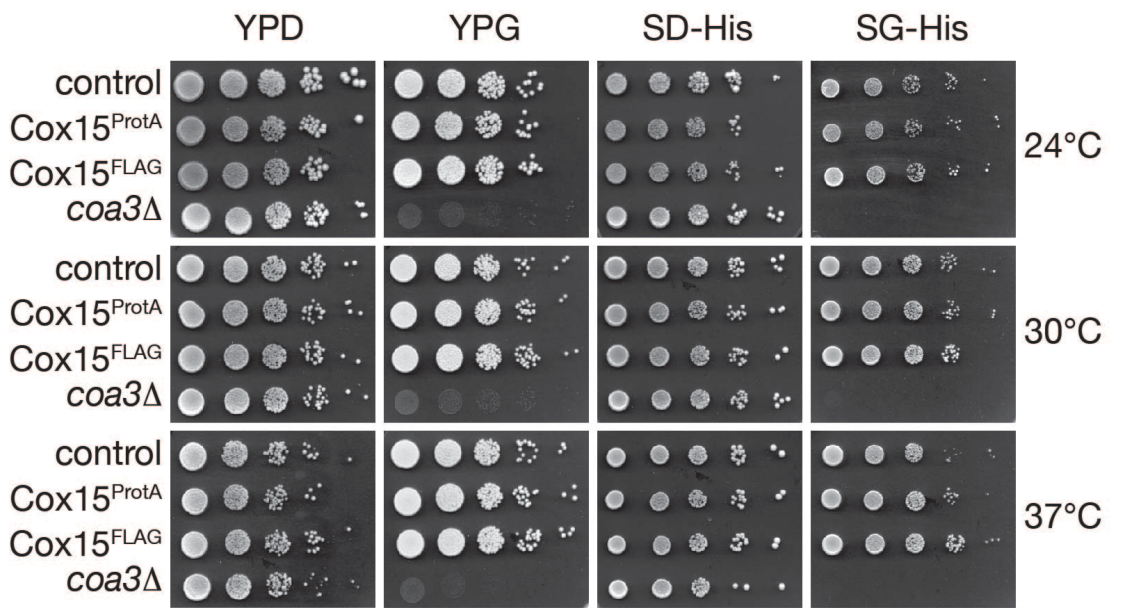

FIG 3.14 Analysis of growth behavior of strains with C-terminally tagged Cox15

Serial dilutions of wild type cells, cells expressing Cox $15^{\text {ProtA }}$ or Cox $15^{\mathrm{FLAG}}$ and coa $3 \Delta$ cells were spotted onto fermentable (YPD) or non fermentable (YPG) solid medium and incubated at indicated temperatures. 


\subsubsection{COA components are not co-isolated with Cox15 ${ }^{\text {ProtA }}$}

Next, we isolated mitochondria from wild type and Cox15 $5^{\text {ProtA }}$ cells, grown on YPG. To confirm the co-purification of Cox15 with Shy1, we performed the reverse experiment and purified complexes via Cox15 $5^{\text {ProtA }}$. After solubilization of mitochondria with

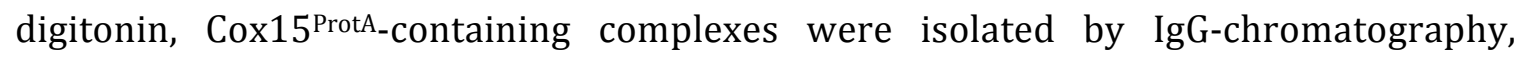
followed by TEV cleavage (FIG 3.15). The native eluates were separated by SDS-PAGE, followed by Western blotting for Cox15, Shy1, COA components and control proteins (FIG 3.15; A). Cox15 ${ }^{\text {ProtA }}$ was isolated efficiently and Shy1 was recovered as suggested by the Shy $1^{\text {ProtA }}$ isolation. However, components of COA complexes, such as Coa1 or Cox14, were not co-purified using Cox15 ProtA.
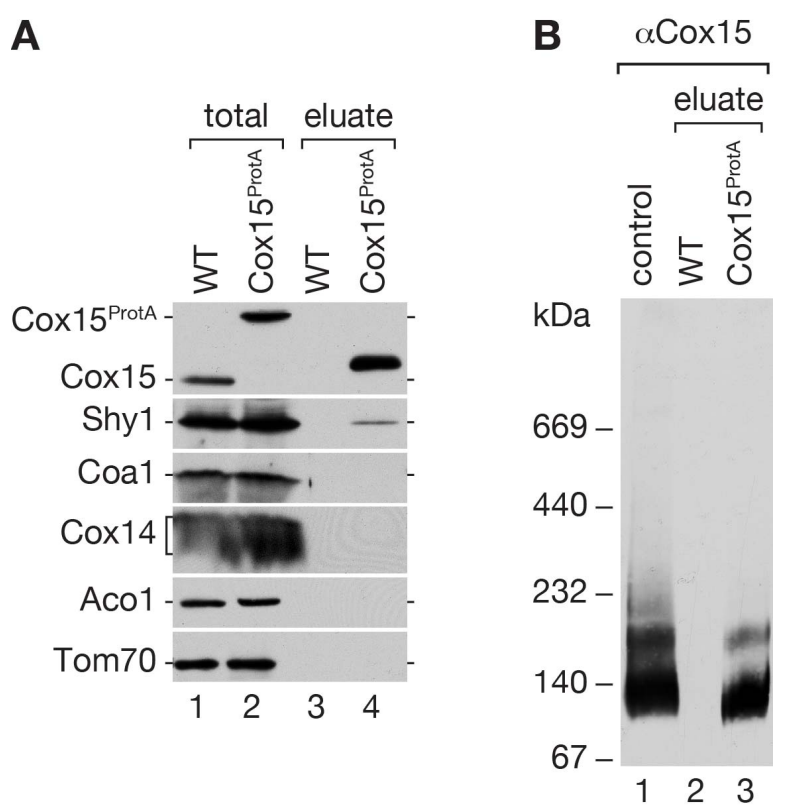

FIG 3.15 Native isolation of Cox15 ProtA interaction partners via IgG-chromatography

(A) Total samples and TEV-cleaved elution of IgG-chromatography from mitochondria, isolated from wild type and Cox $15^{\text {ProtA }}$ were analysed by SDS-PAGE, followed by western blotting. Amounts of protein loaded in the total samples correspond to $6.25 \%$ of the elution. (B) A fraction of native eluted proteins from (A), analysed by $4-13 \%$ BN-PAGE and Western blotting for Cox15 ProtA-containing complexes. As a control, solubilized wild-type mitochondria were loaded ( $9.5 \%$ of eluate).

In addition, BN-PAGE analysis of the eluates revealed a $130 \mathrm{kDa}$ and a $170 \mathrm{kDa}$ complex but no high molecular weight complexes (FIG 3.15; B). This experiment confirmed the interaction of Shy1 and Cox15 seen by mass spectrometry and BN-PAGE. At the same time, the absence of COA complexes contradicted the results obtained by mass spectrometry but is in accordance with previous results, obtained by purification of epitope tagged Cox15 that could not identify Cox15 in complex with Cox1 (Bestwick, Khalimonchuk, et al. 2010b; Khalimonchuk et al. 2012). 


\subsubsection{Co-Immunoprecipitation of Coa3 from wild type vs. Cox15 ${ }^{\mathrm{FLAG}}$}

To clarify the contradictory results on the interaction of Cox15 with COA complexes obtained by different methods, we applied an additional method. Wild type mitochondria were subjected to co-immunoprecipitation with Coa3 or control antibodies in order to isolate COA complexes (Mick et al. 2010). Coa3 antibodies efficiently precipitated COA complexes, indicated by the presence of Shy1, Cox1, Cox5, Mss51, Coa1 and Cox14. In addition, Cox15 was also specifically enriched, providing strong support for the results obtained by mass spectrometry (FIG 3.16; lanes 1-3).

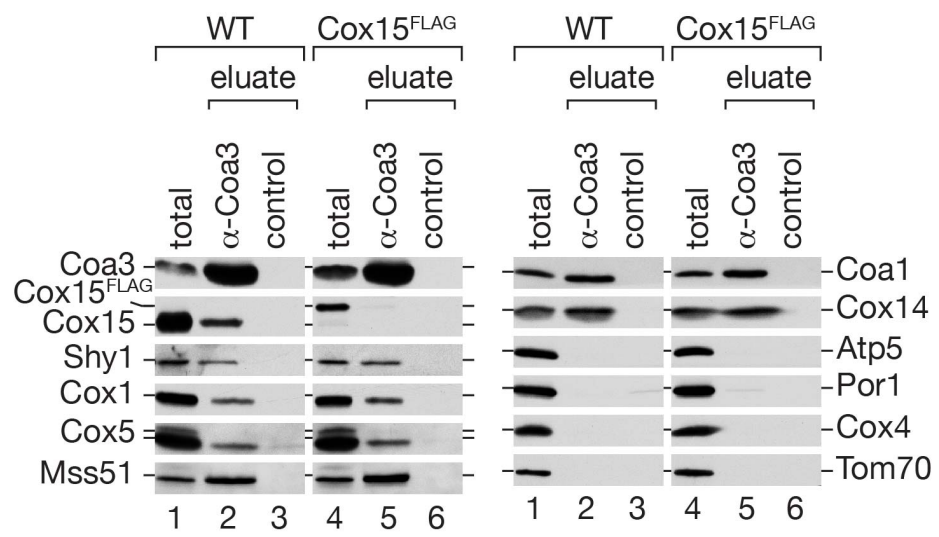

FIG 3.16 Influence of a C-terminal tag on Cox15 on interaction with COA complexes

Co-immunoprecipitation of Coa3 (and control) from digitonin-solubilized mitochondria, isolated from wild type (WT) and Cox15 FLAG. Bound material was eluted and analysed by SDS-PAGE and Western blotting (total 8\%, eluate $100 \%)$.

Control proteins from different mitochondrial compartments were not isolated. We speculated that a C-terminal tag on Cox15 interfered with COA association. In order to address this theory, we performed co-immunoprecipitation from solubilized Cox15 FLAG mitochondria, using Coa3 antibodies (FIG 3.16, lanes 4-6). To avoid the interaction of protein A with antibodies, the tag was replaced by a FLAG tag. COA complex constituents were precipitated with identical efficiency from wild type and Cox15 FLAG mitochondria by Coa3 immuniprecipitation. Cox15, however was only isolated from wild type and was lacking when the precipitation was performed in Cox15 $5^{\text {FLAG }}$. Therefore we concluded that a C-terminal tag on Cox15 affects the stability of the association between Cox15 and COA complexes but not between Cox15 and Shy1 (as seen in FIG 3.15). To circumvent interferences caused by protein tags, the following isolations of assembly intermediates were performed via co-immunoprecipitations against the early assembly factor Coa3 (in some cases also against Coa1). 


\subsubsection{COX assembly in strains with affected heme biogenesis}

\subsubsection{Cox15 enzymatic function is not required for association to COA complexes}

The heme a synthase is a conserved protein across species. A study on the Bacillus subtilis heme a synthase, CtaA, illustrated the importance of 4 highly conserved histidines for catalytical activity by the presence of heme $o$ / heme $a$ in different point mutants. If the residue $\mathrm{H} 216$, that corresponds to $\mathrm{H} 368$ in yeast Cox15, is mutated to methionine, heme $a$ is barely detectable and heme $o$ accumulates (Hederstedt et al. 2005).

In order to study if the association of Cox15 with COA complexes is dependent on its catalytical activity, we transferred this specific mutation into yeast Cox15. FIG 3.17; A shows the region in Cox15 homologues from different species, including B. subtilis. The COX15 gene was cloned into pRS416 under control of its endogenous promotor and site directed mutagenesis was performed. The resulting vector, carrying a H368M exchange in Cox15, was transformed into cox15 cells. For control purposes, an empty plasmid as well as the non-mutated parental plasmid, was transformed. Growth of the strains was analysed on fermentable and non-fermentable carbon source at different temperatures (FIG 3.17; B).

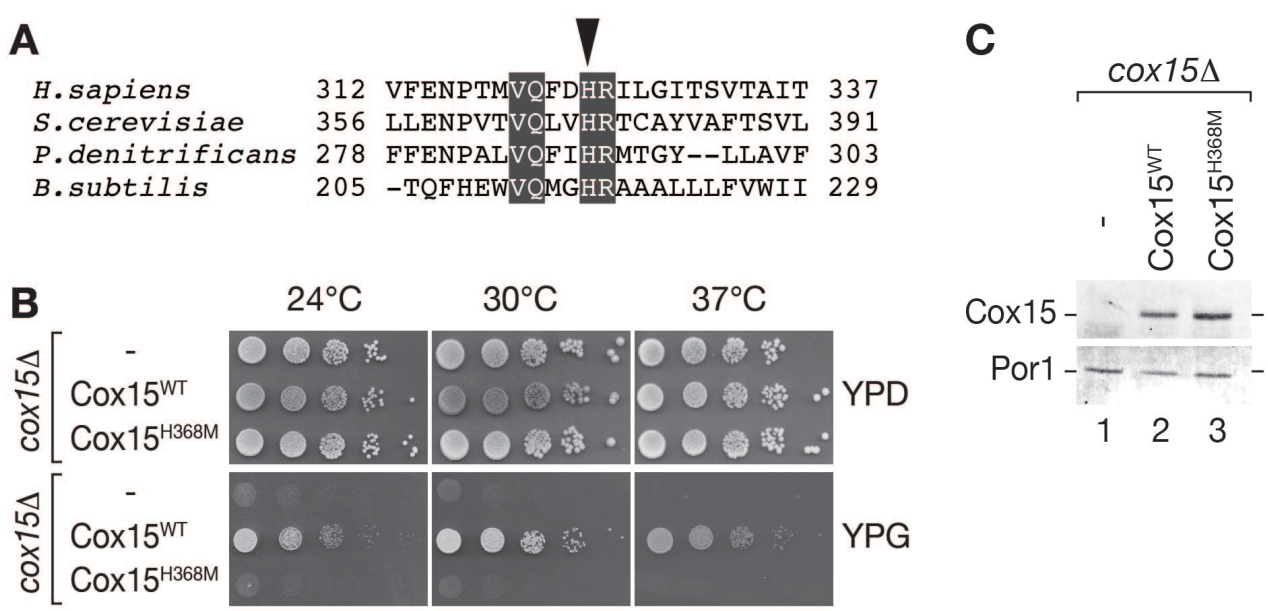

FIG 3.17 Analysis of a Cox15 variant, defective in heme $a$ biogenesis

(A) Alignment of a region in Cox15 homologues from different species (ClustalW2). Black boxes indicate identical residues in all species shown. Arrowhead indicates a conserved histidine at position 216 in B. subtilis,

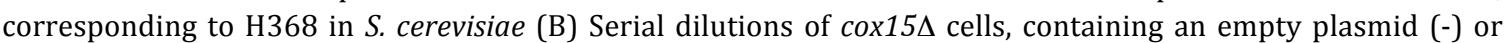
expressing wild type Cox15 (Cox15 ${ }^{\mathrm{WT}}$ ) or a catalytically inactive variant of Cox15 (Cox15 $\left.\mathrm{H}^{\mathrm{H}} 68 \mathrm{M}\right)$ were spotted onto fermentable (YPD) or non-fermentable (YPG) solid medium and incubated at indicated temperatures. (C) Whole cell extracts of strains, described in (B) were analysed by SDS-PAGE and Western Blotting for Cox15 protein levels. Por1 was used as loading control. 
Because the heme $a$ cofactor is essential for cytochrome $c$ oxidase and Cox15 is required for its synthesis, COX15 deletion leads to a deficiency in respiratory growth. Cytochrome $c$ oxidase subunits are present in the cox15 mutant but are not assembled into a functional enzyme (Glerum et al. 1997). In accordance with these findings, cox $15 \Delta$ cells with an empty plasmid were not able to grow under respiratory conditions. As expected, wild type Cox15 was able to rescue this growth phenotype under all tested conditions but Cox15 $15^{\mathrm{H} 368 \mathrm{M}}$ was not. To exclude that the respiratory defect is due to instability of the mutant form of Cox15, Cox15 protein levels were compared in whole cell extracts (FIG 3.17 ; C). We found comparable amounts of wild type and mutant Cox15 protein, detected with Cox15 antibody. No protein was detected in the deletion strain. Equal loading was confirmed using antibodies against mitochondrial porin (Por1).

To confirm the stability of $\operatorname{Cox} 15^{\mathrm{H} 368 \mathrm{M}}$ also in mitochondria, cox $15 \Delta$ cells, carrying an empty plasmid or expressing wild type or mutant Cox15 were grown on SGal-Ura and mitochondria were purified. Different amounts of mitochondria were separated by SDSPAGE, followed by Western blot analysis (FIG 3.18; A). With identical amounts of wild type and mutant Cox15 protein, we confirmed that the stability of Cox15 was not affected by the H368M exchange.

A

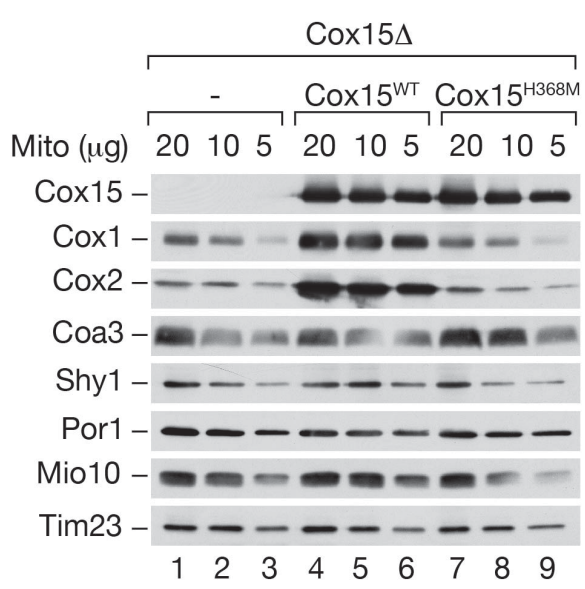

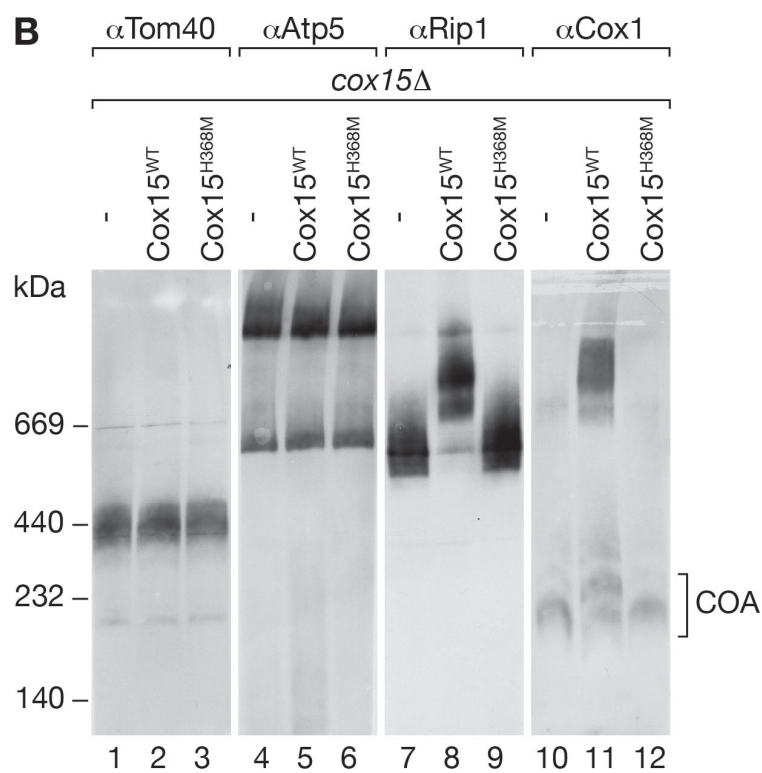

FIG 3.18 Analysis of steady state protein levels of cells expressing Cox15 ${ }^{\mathrm{H}} 36 \mathrm{~m}$

(A) For analysis of steady state protein levels, mitochondria (Mito) isolated from the indicated strains were separated by SDS-PAGE or (B) 4-13\% BN-PAGE and analysed by Western-blotting. 
Cytochrome $c$ oxidase subunits $\operatorname{Cox} 1$ and $\operatorname{Cox} 2$ were reduced in the deletion and mutant mitochondria. However, levels of Coa3 and Shy1 as well as of control proteins were unaffected. Mitochondrial protein complexes were also analysed in these mitochondria by BN-PAGE (FIG 3.18; B). As expected, in $\operatorname{cox} 15 \Delta$, no mature cytochrome $c$ oxidase was detectable (Glerum et al. 1997). In accordance with the growth phenotype and the reduced levels of Cox1 and Cox2, no mature COX was detected in Cox $15^{\text {H368M }}$ mitochondria. Instead, residual Cox1 was found to accumulate in COA complexes.

In $\operatorname{cox} 15 \Delta$ cells containing an empty plasmid or expressing Cox $15^{\mathrm{H} 368 \mathrm{M}}$, other mitochondrial protein complexes, such as the TOM complex (detected with Tom40 antibody) and the $\mathrm{F}_{1} \mathrm{~F}_{0}$-ATP-synthase (detected with Atp5 antibody) were not affected in stability or quantity. Due to the lack of cytochrome $c$ oxidase, the cytochrome $b c_{1}$ complex (detected with Rip1 antibody) was not able to form supercomplexes, and was present only in its dimeric form. However, the total amount of cytochrome $b c_{1}$ complex was similar to wild type. Next, we analysed cytochrome $c$ oxidase assembly intermediates that accumulated in $\operatorname{Cox} 15^{\mathrm{H} 368 \mathrm{M}}$ mitochondria. We addressed the association of wild type Cox15 and Cox15 ${ }^{\text {H368M }}$ with COA complexes by Coa3 immunoprecipitation (FIG 3.19).

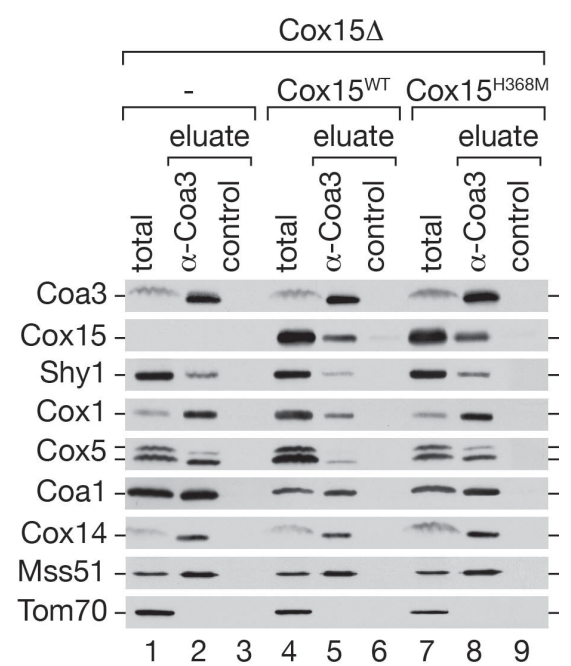

FIG 3.19 Association of Cox15 ${ }^{\mathrm{H} 368 \mathrm{M}}$ with COA complexes

Co-immunoprecipitation of Coa3 (and control) from digitonin-solubilized mitochondria, isolated from cox $15 \Delta$ cells, containing an empty plasmid (-) or expressing Cox15WT or Cox15 ${ }^{\mathrm{H} 368 \mathrm{M}}$. Bound material was eluted and analysed by SDS-PAGE and Western blotting (total 8\%, eluate 100\%). 
Western blot analysis revealed that $\operatorname{Cox} 15^{\mathrm{WT}}$ and $\operatorname{Cox} 15^{\mathrm{H} 368 \mathrm{M}}$ were precipitated with the same efficiency. The small residual amount of Cox1 in the mutant was present in Coa3-containing complexes, together with other COA components (Coa1, Cox14, Cox5, Mss51). These results indicated the accumulation of COA complexes in mitochondria expressing a catalytically compromised Cox15, previously observed during BN-PAGE analysis (FIG 3.18; B). We concluded that Cox15 interaction with Cox1 assembly intermediates occurs independently of its enzymatic function and the final product, heme $a$. 


\subsubsection{Heme o supply by Cox10 is not required for Cox15 interaction with COA complexes}

We stepped one step backwards in the heme biogenesis pathway and asked if Cox15 still interacts with COA complexes in the absence of its substrate heme $o$. To address this question, we generated a $\operatorname{cox} 10 \Delta$ strain. The $\operatorname{COX} 10$ gene encodes the mitochondrial heme o synthase that catalyzes the conversion of protoheme IX to heme $o$ (Tzagoloff et al. 1993), which is further modified by Cox15 to form heme $a$. Since the heme $a$ cofactor is essential to COX catalytical function, deletion or mutation of COX10 leads to a deficiency in respiratory growth. This is caused by the absence of functional cytochrome c oxidase (Nobrega et al. 1990; Tzagaoloff et al. 1993; Valnot et al. 2000a). To confirm this, we purified mitochondria from wild type, $\operatorname{cox} 10 \Delta$ and $\operatorname{cox} 15 \Delta$ and analysed them for cytochrome $c$ oxidase by BN-PAGE and Western blotting, probing against various subunits and an assembly factor of cytochrome $c$ oxidase (FIG 3.20; A).
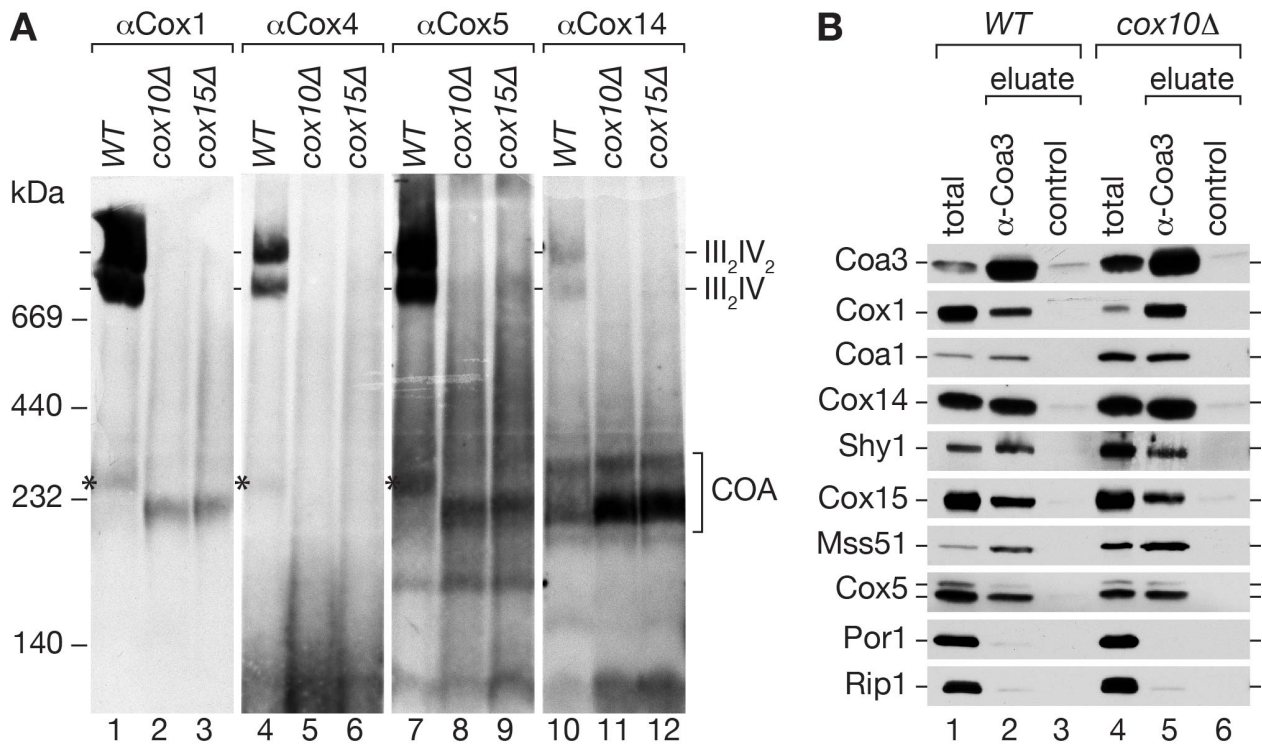

FIG 3.20 Composition of COA complexes in absence of heme $o$ synthase (Cox10) or heme $a$ synthase (Cox15)

(A) $35 u$ digitonin-solubilized wild type (WT), $\operatorname{cox} 10 \Delta$ and $\operatorname{cox} 15 \Delta$ mitochondria separated by 4-13\% BN-PAGE and analysed by Western-blotting for COX subunits Cox 1 , Cox 4 and Cox 5 and the early COX assembly factor Cox14. Asteriks indicates monomeric COX (B) Co-immunoprecipitation of Coa3 (and control) from digitoninsolubilized wild type or cox10 10 mitochondria. Bound material was eluted and analysed by SDS-PAGE and Western blotting (total 8\%, eluate 100\%). 
As expected, in mitochondria isolated from $\operatorname{cox} 15 \Delta$ and $\operatorname{cox} 10 \Delta$ (that lack heme $a$ or heme $a$ and $o$, respectively), no mature cytochrome $c$ oxidase was detected. Instead, on BN-PAGE, an assembly intermediate of cytochrome $c$ oxidase of identical size (around $220 \mathrm{kDa}$ ) accumulated in both strains, represented by detection of the early cytochrome $c$ oxidase subunits Cox 1 and Cox 5 as well as the assembly factor Cox14. The late subunit Cox4, however was not detected in this assembly intermediate (FIG 3.20; A). An intermediate of similar size was found also in the strain that lacked the assembly factor Shy1. In a coa1 $\Delta$ strain, the intermediate complex was also detected but shifted slightly to a smaller size, potentially indicating that the intermediate, described above, contains Coa1 (FIG 3.7).

We speculated that this $220 \mathrm{kDa}$ complex reflects the assembly stage at which the heme $a$ is inserted into Cox1. The fact that the accumulated complex is not shifted in size in

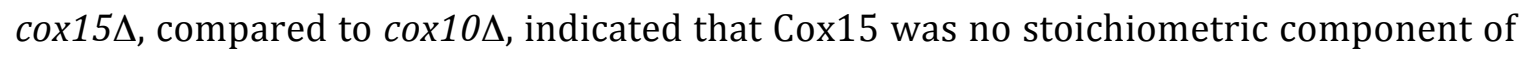
the complex. This finding agreed with the small fraction of Cox15, compared to total levels, that were found to co-isolate with COA complexes (FIG 3.16, lanes 1-3). To determine if Cox15 was associated with COA complexes in $\operatorname{cox} 10 \Delta$, we performed Coa3 immunoprecipitation from these mitochondria (FIG 3.20; B). In $\operatorname{cox} 10 \Delta$, the assembly factors (Coa1, Cox14, Shy1), Mss51, structural subunits (Cox1, Cox5) and Cox15 were efficiently and specifically precipitated by the Coa3 antibody. The small residual amount of Cox 1 in the mutant was present in Coa3 containing complexes, indicating the accumulation of COA complexes in $\operatorname{cox} 10 \Delta$ mitochondria, previously observed with $\mathrm{BN}$ PAGE analysis (FIG 3.20; A). Accordingly, the Cox15 interaction with Cox1 assembly intermediates occured independently of the heme $o$ synthase Cox10 and the substrate of the heme $a$ synthase Cox15, heme $o$. 


\subsubsection{Association of Cox15 and Shy1 with COA complexes}

\subsubsection{Shy1 is not required for Cox15 association to COA complexes}

Shy1 and its homolog SURF1 have been implicated to assist in the heme $a$ insertion into Cox1 (Bundschuh et al. 2009). As we found the terminal enzyme of heme $a$ synthesis, Cox15, to interact with Shy1 and with COA complexes, we asked whether the interaction with COA complexes might be promoted by Shy1. Therefore, we analysed the interaction of Cox15 with COA complexes in absence of Shy1. To address this, we performed Coa3 immunoprecipiation from shy1 $\Delta$ mitochondria, compared to wild type and analysed Cox15 and selected proteins in the eluates by SDS-PAGE and Western blotting (FIG 3.21; A). In shy1s, Cox15 is precipitated with Coa3, together with the assembly factor Cox14, translational regulator Mss51 and early assembling structural subunits of cytochrome $c$ oxidase (Cox1, $\operatorname{Cox} 5, \operatorname{Cox} 6)$. Despite the reduced levels of Cox1 in shy1A, similar amounts were found in COA complexes in wild type and mutant strains. We concluded that Shy1 was not required for the association of Cox15 with COA complexes.
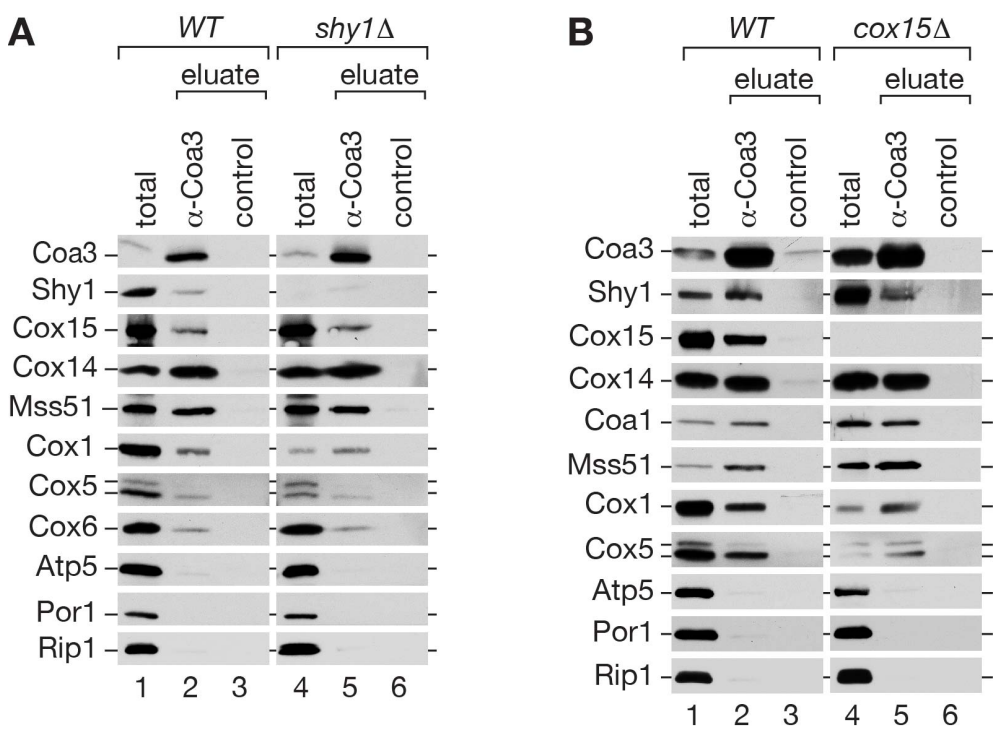

FIG 3.21 Association of Shy1 and Cox15 with COA complexes

(A) Co-immunoprecipitation of Coa3 (and control) from digitonin-solubilized wild type or shy1 $\Delta$ mitochondria. Bound material was eluted and analysed by SDS-PAGE and Western blotting (total 8\%, eluate 100\%). (B) Coimmunoprecipitation of Coa3 from wild type or $\operatorname{cox} 15 \Delta$ mitochondria as in (A). 


\subsubsection{Cox15 is not required for Shy1 association to COA complexes}

As the interaction of Cox15 with COA complexes was not dependent on Shy1, we asked in parallel if the recruitment of Shy1 was dependent on Cox15. Therefore we performed Coa3 immunoprecipitation from wild type and $\operatorname{cox} 15 \Delta$ mitochondria (FIG 3.21; B). In mitochondria lacking Cox15, COA complexes (indicated by the presence of Cox14, Coa1, Mss51, Cox1 and Cox5) were precipitated with identical efficiency, compared to wild type. In analogy to shy $1 \Delta$ cells, Cox1 levels were also severely reduced in cox $15 \Delta$ cell but the residual amount accumulated in COA complexes and was efficiently precipitated. Most interestingly, Shy1 was also co-precipitated from $\operatorname{cox} 15 \Delta$ mitochondria.

We concluded that Cox15 was not necessary for the association of Shy1 with cytochrome $c$ oxidase assembly intermediates. Moreover, Shy1 and Cox15 were able to associate with cytochrome $c$ oxidase assembly intermediates independent of each other. 


\subsubsection{Cox15 associates with COA complexes in absence of Cox1}

Evidence from the bovine cytochrome $c$ oxidase structure (Tsukihara et al. 1995; Tsukihara et al. 1996) suggested that insertion of heme $a$, that is unique to cytochrome $c$ oxidase, into $\operatorname{Cox} 1$ has to occur before the addition of Cox2. We therefore asked if Cox15 interacts with COX assembly factors in the absence of Cox 1 or Cox 2 . To address this question, we isolated mitochondria from cox1- (Netter et al. 1982) and cox2(Kruszewska et al. 1980) cells grown on YPGal. Immunoprecipitation with antibodies directed against Coa3 and Coa1 were performed from digitonin solubilized mitochondria, followed by Western blot analysis for the presence of Cox15 and cytochrome $c$ oxidase assembly factors (FIG 3.22). In the absence of Cox1, Coa3 precipitated Cox14, but in comparison to wild type or cox2', failed to precipitate Cox1, Shy1 or Mss51. Cox15 however was efficiently isolated in the absence of Cox1 and Cox2 (FIG 3.22; A). Similar results were obtained by immunoprecipitation of Coa1 (FIG 3.22; A).

Accordingly, Cox15 was able to interact with Coa3, Coa1 and Cox14 in the absence of Cox1 in a way comparable to wild type. In contrast, the interaction of Shy1 with assembly factors was disturbed in the absence of Cox1.

A
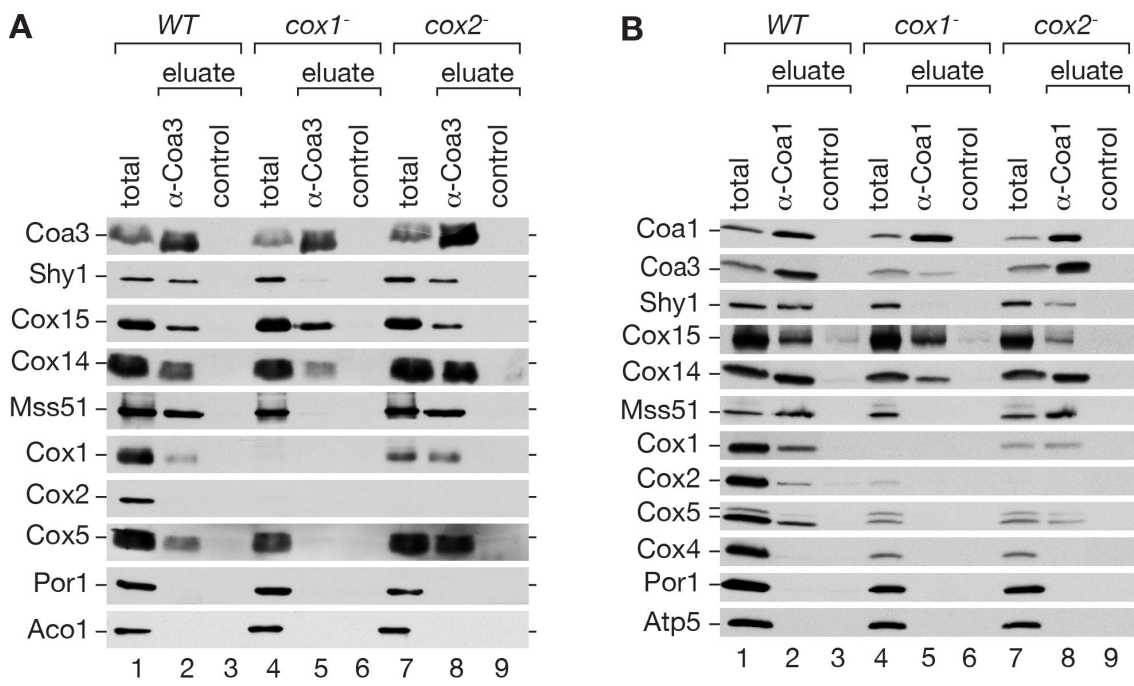

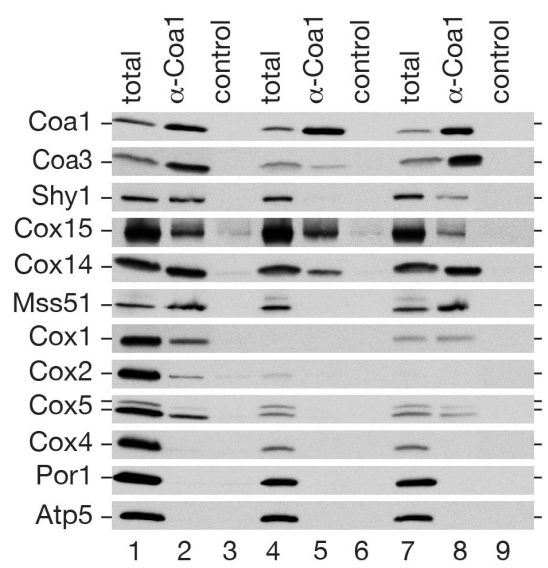

FIG 3.22 Association of Shy1 and Cox15 with COA complexes in absence of Cox1 or Cox2

(A) Co-immunoprecipitation of $\mathrm{Coa} 3$ (and control) from digitonin-solubilized wild type, cox1- or cox2mitochondria. Bound material was eluted and analysed by SDS-PAGE and Western blotting (total 8\%, eluate 100\%). (B) Co-immunoprecipitation of Coa1 as in (A). 


\subsubsection{Cox15 and Shy1 form complexes in absence of mitochondrial DNA}

Next, we analysed the differential behavior of Shy1 and Cox15 in respect to their interaction with assembly factors in a native way. As elution after coimmunoprecipitation was only possible in a denaturing way and due to the lack of genomic markers, chromosomal tagging of Shy1 or Cox15 was not possible in the cox $1^{-}$ and cox2- background. We therefore had to pursue a different experimental strategy. The mutagen ethidium bromide has been used to reduce the mitochondrial copy number (Diaz et al. 2002). By growth of Shy1 $1^{\text {ProtA }}$ cells on media containing EtBr, we generated a strain lacking mitochondrial DNA $\left(r h o^{0}\right)$ and as a consequence also Cox1 and all proteins encoded by the mitochondrial genome. Cox2 however, as previously shown, had no significant effect on the association of Shy1 and Cox15 with COA complexes (FIG 3.22). We isolated mitochondria from wild type, Shy $1^{\text {ProtA }}$ and Shy ${ }^{\text {ProtA, }}$ lacking mitochondrial DNA, grown on YPGal. Solubilized mitochondria were subjected to IgG-chromatography and proteins, eluted with acidic buffer, were analysed by SDSPAGE and Western blotting (FIG 3.23; A).
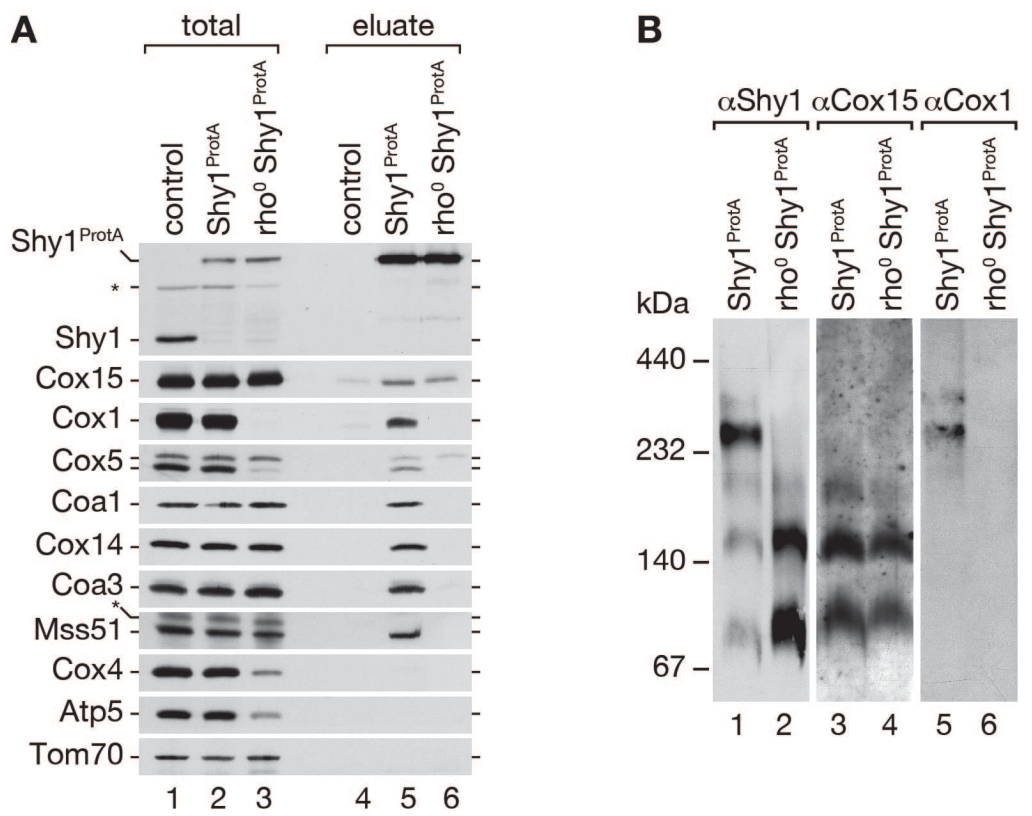

FIG 3.23 Interaction of Shy1 and Cox15 in the absence of mitochondrial DNA (rho $\left.{ }^{\circ}\right)$

(A) Purification of Shy1 ProtA containing complexes by IgG-chromatography from wild type mitochondria, compared to mitochondria lacking mitochondrial DNA $\left(r h o^{\circ}\right)$. Total samples and acidic elution were analysed by SDS-PAGE, followed by Western blotting. Amounts of protein loaded in the total samples correspond to 3\% of the elution. Asterisk indicates a cross-reactive signal (B) Purification of Shy1 ${ }^{\text {ProtA }}$ containing complexes by IgGchromatography from strains as in (A). TEV cleaved native eluates were separated by $4-13 \%$ BN-PAGE, followed by Western blotting. 
For analysis of native protein complexes, proteins were cleaved off the resin by TEV protease and subsequently analysed by BN-PAGE and Western blotting (FIG 3.23; B). In the absence of mitochondrial DNA and mitochondria-encoded proteins, Cox15 was still detectable after Shy $1^{\text {ProtA }}$ isolation. As expected, in contrast to wild type, no components of COA complexes were copurified from $r h o^{\circ}$ cells. When the native eluate of IgGchromatography was analysed by BN-PAGE, we observed Shy1 in distinct complexes and Cox15 in complex with Shy1, predominantly at smaller size but also in higher molecular weight COA complexes, as previously seen (FIG 3. 13). In contrast in Shy1 $1^{\text {ProtA, }}$, lacking mitochondrial DNA, only the two smaller complexes were isolated and contained Cox15.

Taken together, the association of Shy1 and Cox15 occurred independently of Cox1 (or mitochondrial DNA). In addition, Cox15 was able to bind Coa3 and Coa1 in the absence of Shy1 and Cox1. 


\section{DISCUSSION}

In this study, different stages in the early cytochrome $c$ oxidase assembly steps were analysed. We started with the membrane insertion of the central subunit Cox1 by the Oxa1 insertase. Next, isolations of interaction partners of Mss51 were used to address the feedback regulation mechanism of COX1 translation. Furthermore, the dual functionality of Shy1 was dissected with the help of a Leigh syndrome patient SURF1 mutation, transferred to yeast Shy1. Finally assembly intermediates containing the assembly factor Shy1 were characterized in an unbiased manner. This revealed the unexpected interaction of the heme $a$ synthase Cox15 with early COX intermediates that allowed speculations on the heme transfer mechanism and timing during Cox1 maturation.

\subsection{Oxa1-Ribosome complexes coordinate the assembly of cytochrome $c$ oxidase}

0xa1 plays an early role in cytochrome c oxidase biogenesis: The three core subunits of cytochrome c oxidase (Cox1, $\operatorname{Cox} 2$ and $\operatorname{Cox} 3$ ) are synthesized on mitochondrial ribosomes and inserted into the inner membrane in a co-translational reaction facilitated by the Oxa1 insertase (He \& Fox 1997; Hell et al. 2001). In this study, we characterize Oxa1 variants with flexible linker regions of 100 or 200 amino acids, inserted between the $\mathrm{N}$-terminal insertase domain and the C-terminal ribosomebinding region. These mutants, in the combination with the absence of the ribosome receptor Mba1, show reduced cytochrome $c$ oxidase activity, caused by the reduction of Cox1 steady-state levels and cytochrome $c$ oxidase-containing supercomplexes (Keil et al. 2012). As membrane insertion of mitochondrial translation products remained unaffected we concluded that the defects observed in the linker mutants are not caused by a block in membrane insertion but rather occur further downstream in the assembly process of cytochrome $c$ oxidase. 
Interestingly, several factors involved in cytochrome $c$ oxidase assembly or biogenesis have previously been shown to bind to mitochondrial ribosomes:

Cox11, the protein required for copper delivery to newly synthesized Cox1, associates with the mitochondrial translation machinery (Khalimonchuk et al. 2005).

Mdm38, an inner membrane protein, cooperates with Mba1 in ribosome binding. Moreover, Mdm38 is a critical regulatory factor of the synthesis and assembly of mitochondrial translation products (Frazier et al. 2006; Bauerschmitt et al. 2010). Therefore, simultaneous loss of Mba1 and Mdm38 leads to severe defects in the biogenesis of respiratory chain complexes III and IV, not caused by disturbed membrane binding of the mitochondrial ribosome but rather by misregulation of synthesis of cytochrome $b$ (Cob) or Cox1, respectively (Bauerschmitt et al. 2010).

Mss51, a protein loosely associated with the matrix side of the inner membrane, interacts with mitochondrial ribosomes and initiates COX1 translation (Perez-Martinez et al. 2003; Barrientos et al. 2004). Additionally, Mss51 serves as a chaperone binding directly to newly synthesized Cox1 and to the assembly factors Cox14, Coa1, Coa3, and Shy1 (Pierrel et al. 2007; Mick et al. 2010; Fontanesi et al. 2011). This dual functionality of Mss51 directly couples synthesis of Cox1 to assembly of cytochrome $c$ oxidase (PerezMartinez et al. 2009). These examples illustrate that the mitochondrial ribosome is able to serve as a binding platform for COX assembly factors. In yeast, several feedback mechanism regulating translation of mitochondria-encoded subunits of respiratory chain complexes have been described (Fontanesi 2013). In the case of cytochrome $c$ oxidase, translation of the central subunit Cox1 is regulated by Mss51 in dependence of the assembly state of newly synthesized Cox1 (Perez-Martinez et al. 2003; Barrientos et al. 2004; Perez-Martinez et al. 2009). In this context, it is reasonable to tether the ribosome close to the site of cytochrome $c$ oxidase assembly, the mitochondrial inner membrane.

In mba1 $1 \Delta$ cells expressing $0 x 1^{100}$ or $0 x a 1^{200}$, the tight contact of ribosomes with the membrane is lost. This may impair the function of different assembly factors and therefore efficiency of cytochrome c oxidase assembly is decreased. Thus, the close proximity of Oxa1 to the ribosome is not only important for the membrane insertion but also for cytochrome $c$ oxidase biogenesis. We speculate that the spatial proximity of mitochondrial ribosomes to the insertion site in the inner membrane, mediated by Oxa1, allows the binding of assembly factors required for biogenesis of cytochrome $c$ oxidase (FIG 4.1). 

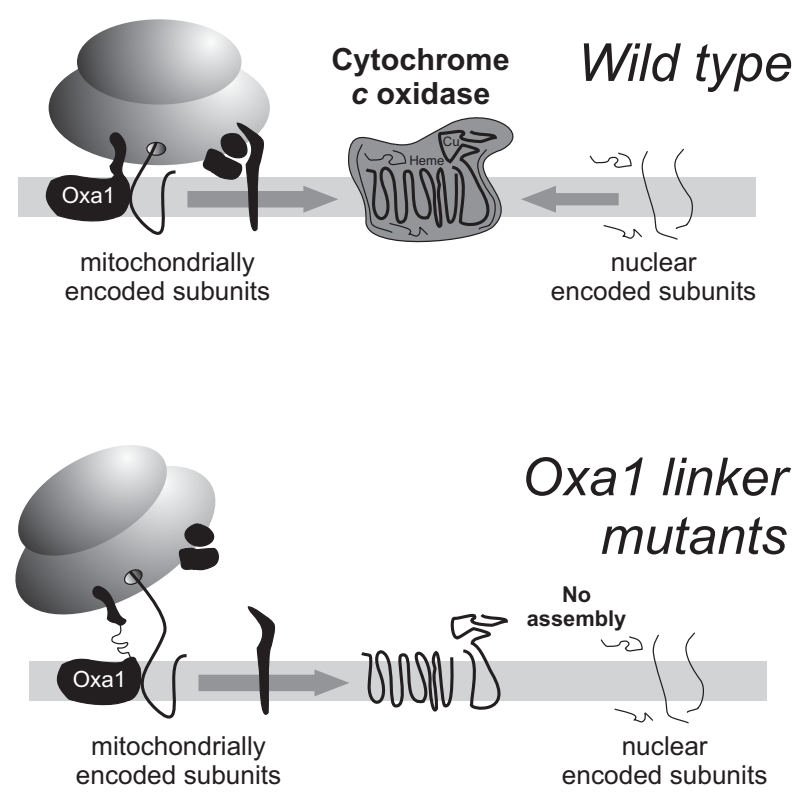

FIG 4.1 Mitochondrial ribosomes serving as a binding platform for COX assembly factors

Spatial proximity of mitochondrial ribosome with the inner membrane enables assembly factors to bind to the sites at which newly synthesized proteins are inserted into the membrane by 0xa1 (upper panel). In the lower panel, the membrane-embedded insertase region and the ribosome-binding region of Oxa1 are spatially separated by a flexible linker. The proposed coordination of assembly factors is interfered and therefore assembly of cytochrome $c$ oxidase is disturbed. Figure adapted from (Keil et al. 2012).

In contrast to Oxa1, YidC, the bacterial member of the conserved YidC/Alb3/Oxa1 protein family, lacks a ribosome-binding domain (Wang \& Dalbey 2011). According to our results, one might speculate that this region has been added to Oxa1 during evolution for two distinct reasons. First, the additional ribosome-binding domain evolved to improve co-translational insertion of membrane proteins. Second, the positioning of the mitochondrial ribosome to the site of protein insertion enables the ribosome to serve as binding platform for assembly factors. As mitochondrial ribosomes, compared to their bacterial counterparts, only have a very limited number of proteins to synthesize (8 in S. cerevisiae, 13 in human), they were potentially able to adopt this additional function.

Taken together, we report Oxa1-Ribosome complexes to coordinate the assembly of cytochrome $c$ oxidase (Keil et al. 2012). Further characterization of the structure and function of these mitochondrial ribosome assembly complexes, e.g. by identification of exact interaction surfaces using crosslinking techniques, will be exciting. 


\subsection{Interaction partners of Mss51 in $\operatorname{coa} 1 \Delta$}

Mss51 is a protein that fulfills dual functions. On the one hand it acts as a translational activator (Perez-Martinez et al. 2003; Zambrano et al. 2007). On the other hand, Mss51 is involved in cytochrome $c$ oxidase assembly, together with other assembly factors. By these dual activities, Mss51 is able to regulate COX1 translation in response to the assembly state of Cox1 via a feedback regulatory mechanism (Barrientos et al. 2004; Perez-Martinez et al. 2009). Furthermore, the protein associates with newly synthesized Cox1 and early assembly factors, such as Coa3, Cox14, Coa1 and Shy1 (Barrientos et al. 2004; Mick et al. 2007; Pierrel et al. 2007; Perez-Martinez et al. 2009; Mick et al. 2010; Reinhold et al. 2011; Fontanesi et al. 2011). The latter interaction transfers Mss51 into an sequestered inactive state. In coa1s mitochondria, Mss51 accumulates in one prominent assembly intermediate complex with Cox1 (around $220 \mathrm{kDa}$, compare FIG 3.6; B) in its sequestered, but active form. As a consequence, Mss51 is not able to shut down COX1 translation despite a defect in the cytochrome $c$ oxidase assembly process (Khalimonchuk et al. 2010; Mick et al. 2010). In order to identify interaction partners of Mss51 in the described complex, Mss51 ${ }^{\mathrm{SF}}$ was purified from coa1 $\Delta$ mitochondria under native conditions. Yeast cells were grown under SILAC conditions (see section 2.9) in order to obtain quantitative data. Mass spectrometry analysis reveals Cox1, Ssc1, Shy1, Cox5a, Cox6 and Oms1 to interact with the bait protein. An interaction of the small COX assembly factors Coa3 and Cox14 was not revealed with the SILAC analysis. However, Coa3 and Cox14 were confirmed to interact with Mss51 by Western blot analysis.

Cox1 is found to interact with Mss51 which is in line with the current model of cytochrome $c$ oxidase assembly, where Cox 1 forms the core of assembly intermediates (e.g. reviewed in Mick et al. 2011). Also in accordance with this model, Mss51 is known to be associated with Cox1 during early assembly steps in order to sequester Mss51 (especially before Coa1 is associated to the assembly intermediate). In coa1s mitochondria, Mss51 can not be released from the $220 \mathrm{kDa}$ complex and assembly intermediates accumulate (Khalimonchuk et al. 2010; Mick et al. 2010). 
It is very much under debate whether Shy1 and Mss51 can be found in one complex. Several publication disclaimed this fact by density gradient experiments (Barrientos et al. 2002; Fontanesi et al. 2010; Khalimonchuk et al. 2010). However, other publications applying other methods could show a clear copurification of Mss51 and Shy1 (Mick et al. 2007; Mick et al. 2010). With this SILAC analysis, we present further evidence to support the fact that Shy1 and Mss51 indeed are found in one complex. Nevertheless, it is very surprising to find Shy1 in a complex with Mss51, isolated from coa1s mitochondria: Coa1 was believed to promote the recruitment of Shy1 to Cox1, contributing to the release of Mss51 from the COA complex (Pierrel et al. 2007; Khalimonchuk et al. 2010). In contrast to this model, our results suggest that Shy1 is able to associate with early assembly intermediates independent of the presence of Coa1.

Ssc1 was described before to interact with Mss51 (Krogan et al. 2006) and Mss51containing complexes (Fontanesi et al. 2010) which is confirmed by the presented results. However, Fontanesi and coworkers (2010) found Ssc1 to be in one complex with Mss51 alone and in a further complex with Mss51, Cox14 and Cox1. In contrast, our data suggest that Ssc1 is present in an additional complex with Cox1 and early assembly factors (Coa3 and Cox14, as confirmed by Western blotting) that also contains Shy1, Cox5a and Cox6. 


\subsection{New order in the assembly process of COX: Cox5a and Cox6}

To our surprise we find Cox5a and Cox6 as part of the $220 \mathrm{kDa}$ complex isolated by Mss51 from coa1 $\Delta$ mitochondria. Although it is known that Cox5a and Cox6 are the first nuclear-encoded subunits that are added to the maturing cytochrome $c$ oxidase, it was believed that this event takes place at a downstream stage of the assembly process. Cox5a and Cox6 form a complex which is necessary for the stabilization of Cox5a. In cox6 6 , only minor amounts of Cox5a remain detectable (Glerum \& Tzagoloff 1997; Church et al. 2005). According to our findings, the Cox5a/Cox6 module is added to the cytochrome $c$ oxidase assembly complex even before Coa1 and the release of Mss51. Analysis of import and assembly of radiolabeled Cox5a into coa1s mitochondria confirms that Cox $5 \mathrm{a}$ is a component of the accumulating COA complex of about $220 \mathrm{kDa}$ (FIG 3.6).

Therefore, we propose a novel order in the COX assembly process: after newly synthesized Cox1 is bound by Coa3, Cox14 and Mss51, Shy1 and the Cox5a/Cox6 module is recruited to the complex, independent of the action of Coa1. Moreover, Coa1 is not sufficient but rather requires the presence of the Cox $5 \mathrm{a} / \operatorname{Cox} 6$ module in order to recruit Shy1 to COX assembly intermediates. The finding that the addition of Cox6 is a key step in the release of Mss51 from assembling complexes is further supported by a comparison of Cox1 synthesis rates in a set of cytochrome $c$ oxidase mutants. ShinguVazquez and co-workers (2010) showed that deletion of the nuclear COX6 gene causes the greatest reduction of mitochondrial translation of Cox1, compared to other assembly factors, such as Coa1 (Shingú-Vázquez et al. 2010). 


\subsection{0ms1: a new player in CoX assembly?}

Oms1 is an integral inner mitochondrial membrane protein with a conserved methyltransferase motif, exposed to the IMS. Oms1 has been implicated in respiratory chain function as it was found to act as a multicopy suppressor of respiratory defects, caused by OXA1 mutations (Lemaire et al. 2004). However, we identified an interaction of Oms1 with the translational activator Mss51. The interaction of 0ms1 with Mss51 could be confirmed by Oms1 $1^{\text {FLAG }}$ isolation (data not shown). In initial growth tests, oms $1 \Delta$ cells show no respiratory defect on non-fermentable full media, but if challenged by growth on minimal media, growth defects of oms $1 \Delta$ become visible at $24{ }^{\circ} \mathrm{C}$ and $37^{\circ} \mathrm{C}$. If mitochondria prepared from oms $1 \Delta$ cells (grown on YPGal at $30^{\circ} \mathrm{C}$ ) are analysed on a molecular level, reorganization of the respiratory chain supercomplexes, containing COX can be observed (data not shown). For a long time, Mss51 was speculated to undergo some still undefined modification for regulation of its activity. It is therefore tempting to speculate that 0ms1 might be involved in the modification of Mss51. However, several facts argue against this theory. First of all, the nature of the modification has to be elucidated. As Oms1 contains a conserved methyltransferase domain, methylation of Mss51 is most likely. However, Mss51 is so far not shown to be modified, neither by methylation nor by another type of modification. Furthermore, the methyltransferase domain of Oms1 is localized to the IMS (Lemaire et al. 2004), but Mss51 is found in the mitochondrial matrix (Barrientos et al. 2004). However, the topology of 0ms1 awaits confirmation. If 0ms1 would be responsible for the modification of the translational activator Mss51, a change in mitochondrial translation, particularly in Cox1, would be expected. However, mitochondrial translation remains unaltered upon deletion of $O M S 1$ (at $30^{\circ} \mathrm{C}$, data not shown). In conclusion, most evidence speaks against Oms1 being the factor responsible for the regulation of Mss51 activity by methylation. Nevertheless, taken the genetic interaction with OXA1 and the physical interaction with Mss51, a role of Oms1 in the biogenesis of cytochrome $c$ oxidase in the widest sense is highly likely. More evidence is required to understand the exact molecular function of Oms1. In order to clarify this, mitochondria isolated from oms $1 \Delta$ cells grown on minimal media at non-permissive temperature are interesting to analyse. 
In addition, an antibody against $0 \mathrm{~ms} 1$ has to be generated to be able to detect the endogenous protein by Western blot analysis and to perform immunoprecipitation or antibody-shift assays. In parallel, import of radiolabeled Oms1 into isolated mitochondria and assembly into potential assembly intermediates or even the mature oxidase can be analysed. Furthermore, epitope tagged 0ms1 can be isolated in a more preparative way in order to identify interacting proteins by a combination of mass spectrometry and Western blotting, as it was done for Shy1-containing complexes in this study.

\subsection{Accumulation of COA complexes in a $S H Y 1$ mutant}

Leigh syndrome, a fatal encephalomyopathy, is often associated with cytochrome $c$ oxidase deficiency. Multiple mutations in SURF1, an assembly factor of COX, are described to cause Leigh syndrome (Péquignot et al. 2001; Shoubridge 2001). In this study, we use the yeast homolog of SURF1, Shy1, to study defects in COX assembly observed in Leigh syndrome patients. Therefore, a missense mutation of a conserved amino acid that is reported from Leigh syndrome patients was transferred to the yeast

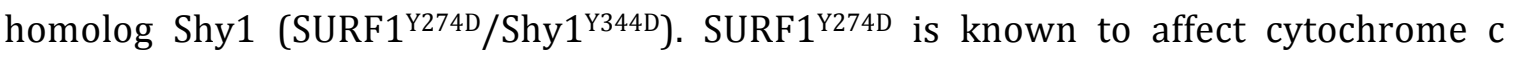
oxidase biogenesis (Teraoka et al. 1999). Yeast strains expressing the corresponding mutant Shy $1^{\mathrm{YD}}$ display a cold sensitive growth phenotype on non-fermentable media. As a consequence of defective COX assembly, levels of mature COX and of mitochondriaencoded subunits Cox 1 and Cox 2 are severely reduced. Synthesis of Cox1 occurs at same rates in wild type and mutant strains, however newly synthesized Cox1 undergoes rapid degradation in the strain, expressing Shy $1^{\mathrm{YD}}$ (Reinhold et al. 2011). The degradation of misfolded or in this case, unassembled proteins is mediated by mitochondrial inner membrane m-AAA proteases (Guzélin et al. 1996; Arlt et al. 1998). Therefore, we conclude that in yeast cells, expressing Shy1 ${ }^{\mathrm{YD}}$, COX1 translational regulation is uncoupled from cytochrome $c$ oxidase assembly. Similarly, the Y274D exchange in SURF1 alters the assembly process of cytochrome $c$ oxidase, leading to the accumulation of an COX assembly intermediate of $200 \mathrm{kDa}$ (Reinhold et al. 2011). In order to elucidate the reason behind this uncoupling phenomenon in yeast, COA complexes were isolated by Coa3-immunoprecipitation. By analysis of co-purified proteins by SDS-PAGE, no accumulation of components of COA complexes was observed (Reinhold et al. 2011). However, in this study, COA complexes are isolated and analysed in their native state by BN-PAGE. 
In contrast to previous analysis, accumulation of a complex, containing Shy $1^{\mathrm{YD}}$, Cox1 and Mss51 was observed. Similar assembly intermediates containing Mss51 and Cox1 accumulate in shy1 $\Delta$ mitochondria (Pierrel et al. 2007). Shy1 fulfills a dual role in COX assembly by releasing the COX1 translational block and as an assembly factor, potentially in heme insertion into newly synthesized Cox1 (Mashkevick et al. 1997; Mick et al. 2007; Bundschuh et al. 2009). The cooperation of Shy1 and Mss51 is of importance for the negative feedback regulation mechanism of COX1 translation (Perez-Martinez et al. 2009). Under regular circumstances, this mechanism prevents the accumulation of unassembled Cox1 species that can potentially act as pro-oxidants, generating reactive oxygen species (Khalimonchuk et al. 2007). Taken together, Shy $1^{\mathrm{YD}}$ is obviously still able to conduct one of its functions as mitochondrial translation rates are comparable to wild type mitochondria. On the other hand, Shy $1^{\mathrm{YD}}$ is no longer able to promote Cox1 maturation. We speculate that Mss51 indeed is sequestered in COA complexes, containing Shy1 ${ }^{\mathrm{YD}}$. Despite the successful sequestration, the mutant form of Shy1 is not able to transform Mss51 into its inactive form. Thus, Mss51 is still able to initiate further rounds of Cox1 synthesis although COX assembly can not progress. These results also explain the molecular mechanism behind the defects leading to Leigh syndrome in patients carrying the analysed Y274D mutant version of SURF1. SURF1 ${ }^{\mathrm{YD}}$ is efficiently imported into mitochondria and, in contrast to other described missense mutations, remains stable. Despite these facts, cells expressing SURF1 ${ }^{\mathrm{YD}}$ exhibit severe defects in COX assembly that lead to the subsequent accumulation of SURF1 ${ }^{\mathrm{YD}}$ in assembly intermediates (Reinhold et al. 2011). However, even in the absence of SURF1, up to $20 \%$ residual cytochrome $c$ oxidase activity is observed in Leigh syndrome patients (Tiranti et al. 1998), indicating that assembly of the cytochrome $c$ oxidase is still possible to some extent. 


\subsection{The heme $a$ synthase Cox15 associates with cytochrome $c$ oxidase assembly intermediates during Cox1 maturation}

During assembly of cytochrome c oxidase, prosthetic groups have to be inserted into Cox1 and Cox2 (Tsukihara et al. 1995; Soto et al. 2012). Although these non-protein cofactors are essential for the catalytic activity of the enzyme, little is known about at which step of the assembly process the insertion occurs. Cox1, the core subunit of the cytochrome $c$ oxidase, receives two heme a cofactors during its maturation. After combined mass spectrometry and Western blot analysis of Shy1-containing assembly intermediates, we unexpectedly found the heme $a$ synthase Cox15 to form complexes with Shy1 and to associate with cytochrome c oxidase assembly intermediates (Bareth et al. 2013). The heme $a$ synthase Cox15 catalyzes the last step of heme $a$ synthesis from heme $o$ (Tzagoloff et al. 1993). Shy1/SURF1 is involved in Cox1 maturation, serving as an assembly factor of cytochrome $c$ oxidase (Nijtmans et al. 2001; Mick et al. 2007). A recent in vitro study with Surf1 (a homolog of yeast Shy1) from the bacterium P. denitrificans indicates its ability to bind heme $a$. This finding linked Surf1 function to the heme incorporation into Cox1 during cytochrome $c$ oxidase biogenesis (Bundschuh et al. 2009). Therefore, Surf1 is proposed to act as a mobile heme carrier between the heme $a$ synthase and Cox1. In contrast, our results in yeast indicate that Cox15 is able to associate with Cox1-containing complexes independent of Shy1. We conclude that Shy1 does not act as a mobile heme carrier, as proposed earlier, but rather teams up with Cox15 in order to transfer and insert heme $a$ into maturing Cox1 in early assembly intermediates (FIG 4.2).

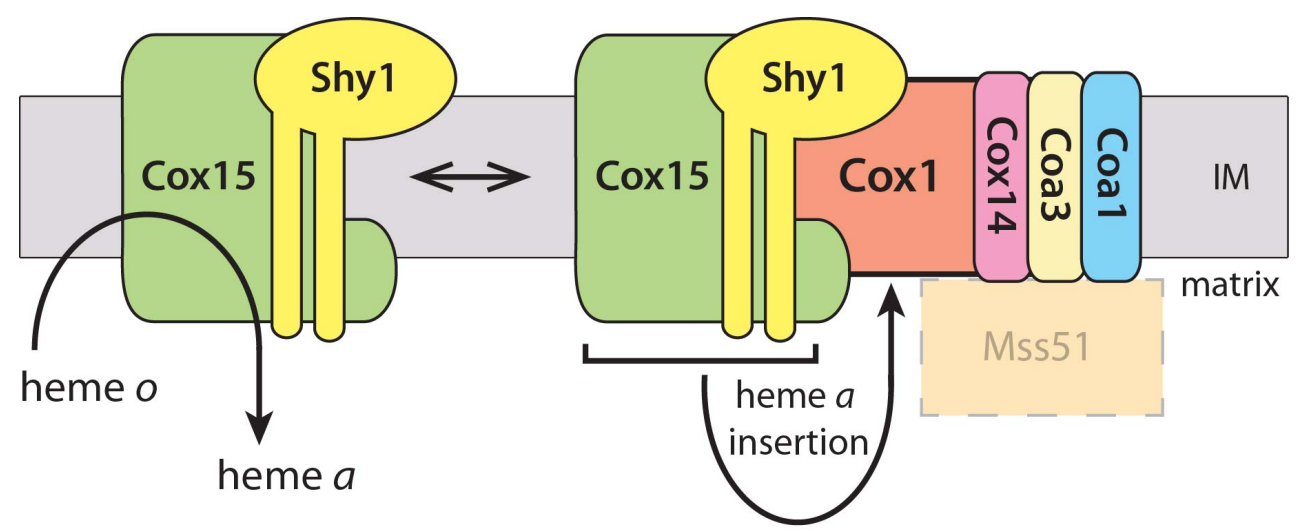

FIG 4.2 The heme $a$ synthase Cox15 forms complexes with Shy1 and associates with COA complexes Cox15 catalyzes the last step of the biogenesis of heme $a$, an essential cofactor of COX. Cox15 is found in native complexes with Shy1 alone but also in COA complexes, together with assembly factors Coa3, Cox14, Coa1 and Shy1. This complex reflects the potential stage of heme insertion into maturing Cox1. Association of Mss51 with Cox15-containing COA complexes was not specifically addressed. Inner mitochondrial membrane (IM). 
In addition, the presence of Shy1 and Cox15 in COA complexes allows to conclude that the last step of heme $a$ synthesis occurs in close proximity to the site of insertion into the target protein, Cox1. This coupling would minimize the risk of damages to the cell caused by the release of a highly reactive free heme moiety. In this context, not only a spatial but even a timed coordination of both processes is imaginable.

\subsection{Association of Shy1 and Cox15 with COA complexes}

Surprisingly, the association of Cox15 with COA complexes is independent of Shy1. In shy1s, Cox15 is in complex together with Coa3, Cox14, Coa1 and with Cox1, the heme receiving protein. This finding is especially interesting as it explains residual COX activity found not only in shy1 $1 \Delta$ yeast mitochondria but also in Leigh syndrome patients with SURF1 mutations (Tiranti et al. 1998; Zhu et al. 1998; Barrientos et al. 2002; Mick et al. 2007; Bestwick, Jeong, et al. 2010a;). In order to display COX activity, the essential heme groups have to be inserted into Cox1. Our results allow us to speculate that Cox15, in the absence of Shy1/SURF1 is able to perform this insertion, however at drastically reduced efficiency. In contrast, disruption of COX15 or mutations leading to a catalytic inactive Cox15 result in a complete loss of mature cytochrome $c$ oxidase (FIG 3.18 and Glerum et al. 1997; Antonicka et al. 2003). This is due to a lack of the essential cofactor heme $a$ (FIG 4.3). The presented results indicate that enzymatically compromised Cox15 still forms complexes with Shy1 and is associated with COA complexes, indistinguishable from wild type Cox15. Therefore complex formation is not linked to the heme $a$ synthase activity of Cox15. The same is true in the absence of heme $o$, the substrate of the heme $a$ synthase. If the heme $o$ synthase Cox10 is deleted, Cox15 is not able to synthesize heme $a$ but is still found in COA complexes. Taken together, our results indicate that complex formation of Cox15 with COA complexes and therefore the role of Cox15 in COX assembly is not linked to its catalytic activity. Therefore we propose an additional chaperone-like function of Cox15 which is independent of its role in the synthesis of heme $a$. We speculate that Cox15 might not act as heme $a$ synthase but in addition as heme $a$ insertase. 


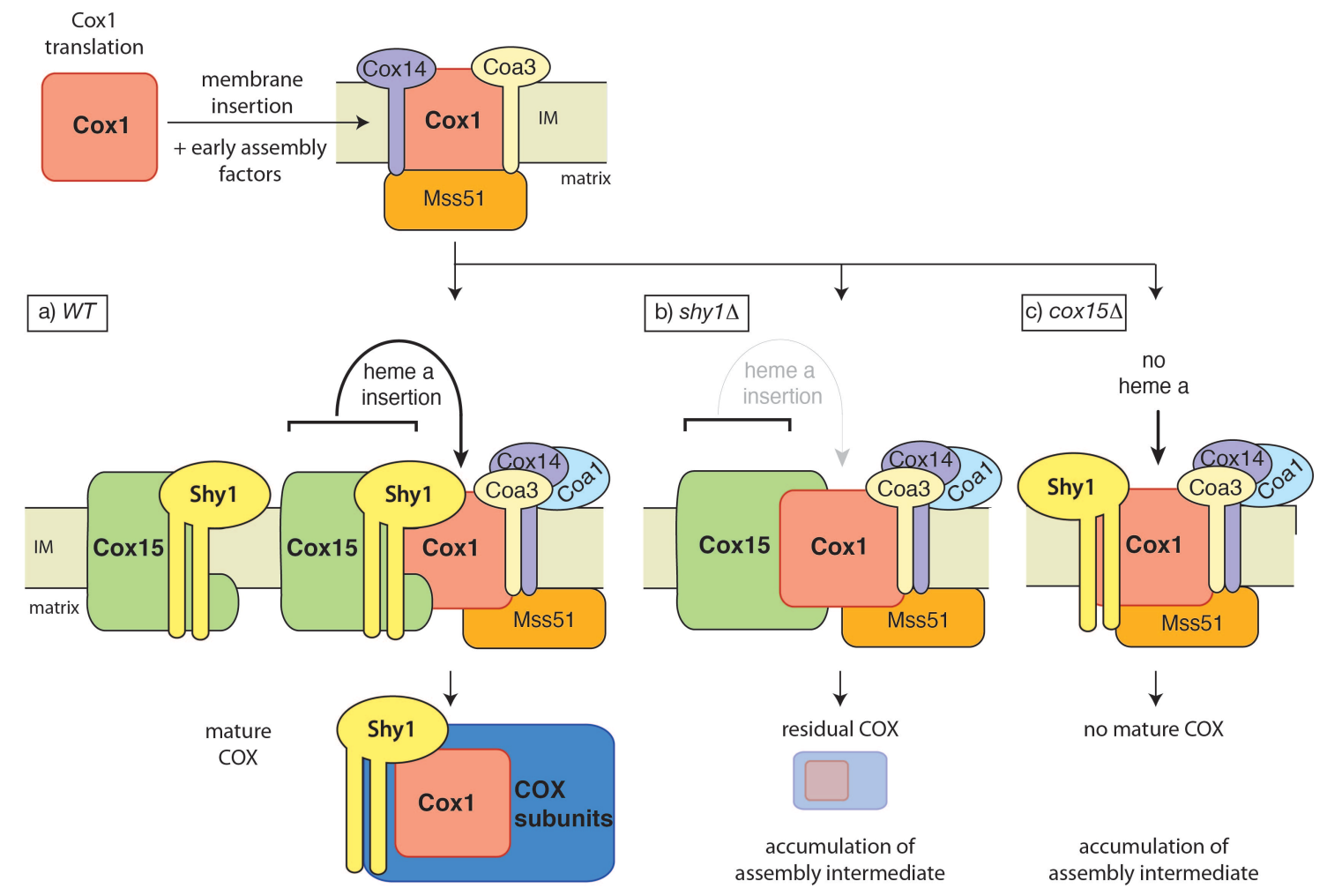

FIG 4.3 Interaction of Cox15 and Shy1 with COA complexes

In wild type, Cox15 interacts with Shy1 and COA complexes, formed after membrane insertion of newly synthesized Cox1. In the absence of Shy1 (b), Cox15 is still found in COA complexes, potentially inserting heme a into Cox1 without the cooperation with Shy1, accounting for residual amounts of active COX. In the absence of Cox15 (c), mature COX is absent due to a lack of essential cofactor heme $a$. 


\subsection{Conclusion}

The results presented in this study add novel aspects that help for a more precise understanding of early steps of cytochrome $c$ oxidase assembly.

i) Oxa1 is involved not only in the membrane insertion of Cox1 but acts also in downstream processes of cytochrome $c$ oxidase assembly. Oxa1 ensures the spatial proximity of mitochondrial ribosomes to the site of membrane insertion at the inner membrane, important for membrane insertion of newly synthesized proteins. Moreover, this Oxa1-ribosome complex coordinates cytochrome $c$ oxidase assembly by providing a platform for binding of assembly factors (Keil et al. 2012).

ii) After membrane insertion of Cox1 by the 0xa1 insertase, early assembly factors such as Coa3 and Cox14 as well as the translational regulator Mss51 associate to Cox1 and form COA complexes. Subsequent binding of Coa1 is believed to recruit Shy1 to the assembly intermediate and Mss51 is sequestered and inactivated in this complex (Mick et al. 2007; Pierrel et al. 2007). However our results propose a novel order in the COX assembly process: after binding of Cox1 by Coa3, Cox14 and Mss51, Shy1 and the Cox5a/Cox6 module are recruited to the complex, independent of the action of Coa1. Moreover, Coa1 is not sufficient for the recruitment of Shy1 to assembly intermediates but rather requires the presence of $\operatorname{Cox} 5 \mathrm{a} / \operatorname{Cox} 6$.

iii) Oms1, a novel interaction partner of Mss51 is identified. Oms1 was originally described as a multicopy suppressor of respiratory defects, caused by a OXA1 mutation (Lemaire at al. 2004). Taken this genetic interaction and the physical interaction with Mss51, Oms1 is an interesting potential new candidate involved in cytochrome $c$ oxidase assembly. Initial results suggest respiratory defects and specific alteration of cytochrome $c$ oxidase containing supercomplexes upon deletion of OMS1, providing direction for future studies. 
iv) Shy1 couples Cox1 translational regulation to COX assembly by a regulatory feedback mechanism in yeast mitochondria (Barrientos et al. 2002; Mick et al. 2007). Defects in cytochrome $c$ oxidase assembly observed in Leigh syndrome patients are studied using use the yeast homolog of SURF1, Shy1. In yeast cells expressing the corresponding patient mutation form (Shy $1^{\mathrm{YD}}$ ), cytochrome $c$ oxidase assembly is disturbed but Cox1 translation rates are comparable to wild type, indicates uncoupling of translational regulation from cytochrome $c$ oxidase assembly (Reinhold et al. 2011). This uncoupling phenomenon can be explained by the accumulation of an assembly intermediate, containing Cox1, Mss51 and Shy $1^{\mathrm{YD}}$. Based on this finding we propose that Shy $1^{\mathrm{YD}}$ is indeed able to sequester but not to transform Mss51 into its inactive state.

v) Biogenesis of mature, catalytic active cytochrome $c$ oxidase requires the incorporation of prosthetic groups into the core subunits Cox1 and Cox2. Upon isolation of Shy1-containing complexes, we find the heme $a$ synthase Cox15 associated with Shy1 and with cytochrome $c$ oxidase assembly intermediates, containing Cox1. This notion allows the speculation that the transfer and insertion of heme $a$ into maturing Cox 1 is facilitated by the cooperated function of Shy1 and Cox15. In addition, the presence of Shy1 and Cox15 in COA complexes suggests that the last step of heme $a$ synthesis occurs in close proximity to the site of insertion into the target protein, Cox1 (Bareth et al. 2013).

vi) The reported interaction of Cox15 with COA complexes persists upon deletion of SHY1. In shy1 $\Delta$ yeast mitochondria as well as in Leigh syndrome patients with SURF1 mutations residual COX activity is reported (Tiranti et al. 1998; Zhu et al. 1998; Barrientos et al. 2002; Mick et al. 2007; Bestwick, Jeong, et al. 2010a;). Therefore we conclude from our results that Cox15, in the absence of Shy1/SURF1 is able to perform insertion of heme $a$ into Cox1, however at drastically reduced efficiency (Bareth et al. 2013). 


\section{BIBLIOGRAPHY}

Acín-Pérez, R. et al., 2008. Respiratory active mitochondrial supercomplexes. Molecular cell, 32(4), pp.529-539.

Adams, K.L. \& Palmer, J.D., 2003. Evolution of mitochondrial gene content: gene loss and transfer to the nucleus. Molecular phylogenetics and evolution, 29(3), pp.380-395.

Alfadhel, M. et al., 2011. Infantile cardioencephalopathy due to a COX15 gene defect: report and review. American journal of medical genetics, Part A, 155A(4), pp.840-844.

Alkhaja, A.K. et al., 2012. MINOS1 is a conserved component of mitofilin complexes and required for mitochondrial function and cristae organization. Molecular biology of the cell, 23(2), pp.247-257.

Altamura, N. et al., 1996. The Saccharomyces cerevisiae OXA1 gene is required for the correct assembly of cytochrome c oxidase and oligomycin-sensitive ATP synthase. FEBS Letters, 382(1-2), pp.111-115.

Antonicka, H. et al., 2003. Mutations in COX15 produce a defect in the mitochondrial heme biosynthetic pathway, causing early-onset fatal hypertrophic cardiomyopathy. The American Journal of Human Genetics, 72(1), pp.101-114.

Arlt, H. et al., 1998. The formation of respiratory chain complexes in mitochondria is under the proteolytic control of the m-AAA protease. The EMBO Journal, 17(16), pp.48374847.

Arnold, I. et al., 1998. Yeast mitochondrial F1F0-ATP synthase exists as a dimer: identification of three dimer-specific subunits. The EMBO Journal, 17(24), pp.71707178.

Bareth, B. et al., 2013. The heme a synthase Cox15 associates with cytochrome c oxidase assembly intermediates during Cox1 maturation. Molecular and Cellular Biology, 33(20), pp.4128-4137

Barrientos, A., Korr, D. \& Tzagoloff, A., 2002. Shy1p is necessary for full expression of mitochondrial COX1 in the yeast model of Leigh's syndrome. The EMBO Journal, 21(12), pp.43-52.

Barrientos, A., Zambrano, A. \& Tzagoloff, A., 2004. Mss51p and Cox14p jointly regulate mitochondrial Cox1p expression in Saccharomyces cerevisiae. The EMBO Journal, 23(17), pp.3472-3482.

Barros, M.H. et al., 2001. Involvement of mitochondrial ferredoxin and Cox15p in hydroxylation of heme 0. FEBS Letters, 492(1-2), pp.133-138.

Barros, M.H. \& Tzagoloff, A., 2002. Regulation of the heme A biosynthetic pathway in Saccharomyces cerevisiae. FEBS Letters, 516(1-3), pp.119-123. 
Barth, P.G. et al., 2004. X-linked cardioskeletal myopathy and neutropenia (Barth syndrome): an update. American journal of medical genetics. Part A, 126A(4), pp.349354.

Bauerschmitt, H. et al., 2010. Ribosome-binding proteins Mdm38 and Mba1 display overlapping functions for regulation of mitochondrial translation. Molecular biology of the cell, 21(12), pp.1937-1944.

Becker, T., Böttinger, L. \& Pfanner, N., 2012. Mitochondrial protein import: from transport pathways to an integrated network. Trends in biochemical sciences, 37(3), pp.85-91.

Beers, J., Glerum, D.M. \& Tzagoloff, A., 1997. Purification, characterization, and localization of yeast Cox17p, a mitochondrial copper shuttle. The Journal of biological chemistry, 272(52), pp.33191-33196.

Bestwick, M., Jeong, M.-Y., et al., 2010a. Analysis of Leigh Syndrome Mutations in the Yeast SURF1 Homolog Reveals a New Member of the Cytochrome Oxidase Assembly Factor Family. Molecular and Cellular Biology, 30(18), pp.4480-4491.

Bestwick, M., Khalimonchuk, O., et al., 2010b. The Role of Coa2 in Hemylation of Yeast Cox1 Revealed by Its Genetic Interaction with Cox10. Molecular and Cellular Biology, 30(1), pp.172-185.

Bohnert, M. et al., 2010. Cooperation of stop-transfer and conservative sorting mechanisms in mitochondrial protein transport. Current biology, 20(13), pp.1227-1232.

Bonnefoy, N. et al., 2009. Roles of Oxa1-related inner-membrane translocases in assembly of respiratory chain complexes. Biochimica et Biophysica Acta - Molecular Cell Research, 1793(1), pp.60-70.

Bowles, E.J., Campbell, K.H.S. \& St John, J.C., 2007. Nuclear transfer: preservation of a nuclear genome at the expense of its associated mtDNA genome(s). Current topics in developmental biology, 77, pp.251-290.

Bradford, M.M., 1976. A rapid and sensitive method for the quantitation of microgram quantities of protein utilizing the principle of protein-dye binding. Analytical Biochemistry, 72, pp.248-254.

Brandner, K. et al., 2005. Taz1, an outer mitochondrial membrane protein, affects stability and assembly of inner membrane protein complexes: implications for Barth Syndrome. Molecular biology of the cell, 16(11), pp.5202-5214.

Brown, K.R. et al., 2002. Identification of novel hemes generated by heme A synthase: evidence for two successive monooxygenase reactions. Biochemistry, 41(36), pp.1090610913.

Brunori, M. et al., 1987. Cytochrome-c oxidase. Subunit structure and proton pumping. European journal of biochemistry / FEBS, 169(1), pp.1-8.

Bundschuh, F.A. et al., 2009. Surf1, associated with Leigh syndrome in humans, is a hemebinding protein in bacterial oxidase biogenesis. Journal of Biological Chemistry, 284(38), pp.25735-25741.

Carr, H.S. \& Winge, D.R., 2003. Assembly of cytochrome c oxidase within the mitochondrion. Accounts of chemical research, 36(5), pp.309-316. 
Castresana, J. et al., 1994. Evolution of cytochrome oxidase, an enzyme older than atmospheric oxygen. The EMBO Journal, 13(11), pp.2516-2525.

Caughey, W.S. et al., 1975. Heme A of cytochrome c oxicase. Structure and properties: comparisons with hemes B, C, and S and derivatives. The Journal of biological chemistry, 250(19), pp.7602-7622.

Chacinska, A. et al., 2009. Importing mitochondrial proteins: machineries and mechanisms. Cell, 138(4), pp.628-644.

Chen, X. et al., 1995. Rearranged mitochondrial genomes are present in human oocytes. The American Journal of Human Genetics, 57(2), pp.239-247.

Chen, Y.-C. et al., 2012. Identification of a protein mediating respiratory supercomplex stability. Cell Metabolism, 15(3), pp.348-360.

Choquet, Y. et al., 2001. Assembly-controlled regulation of chloroplast gene translation. Biochemical Society transactions, 29(4), pp.421-426.

Church, C. et al., 2005. A role for Pet100p in the assembly of yeast cytochrome c oxidase: interaction with a subassembly that accumulates in a pet100 mutant. The Journal of biological chemistry, 280(3), pp.1854-1863.

Clemente, P. et al., 2013. hCOA3 stabilizes COX1 and promotes cytochrome c oxidase assembly in human mitochondria. Journal of Biological Chemistry, 288(12), pp.83218331

Cobine, P.A., Pierrel, F. \& Winge, D.R., 2006. Copper trafficking to the mitochondrion and assembly of copper metalloenzymes. Biochimica et Biophysica acta, 1763(7), pp.759772.

de Brito, O.M. \& Scorrano, L., 2010. An intimate liaison: spatial organization of the endoplasmic reticulum-mitochondria relationship. The EMBO Journal, 29(16), pp.27152723.

Diaz, F. et al., 2002. Human mitochondrial DNA with large deletions repopulates organelles faster than full-length genomes under relaxed copy number control. Nucleic Acids Research, 30(21), pp.4626-4633.

Dimauro, S. et al., 2002. Clinical features and genetics of myoclonic epilepsy with ragged red fibers. Advances in neurology, 89, pp.217-229.

Dimauro, S. \& Davidzon, G., 2005. Mitochondrial DNA and disease. Annals of medicine, 37(3), pp.222-232.

Dimauro, S. \& Schon, E.A., 2008. Mitochondrial disorders in the nervous system. Annual review of neuroscience, 31, pp.91-123.

Dudek, J., Rehling, P. \& Van Der Laan, M., 2013. Mitochondrial protein import: common principles and physiological networks. Biochimica et Biophysica acta, 1833(2), pp.274285.

Dyall, S.D., Brown, M.T. \& Johnson, P.J., 2004. Ancient invasions: from endosymbionts to organelles. Science, 304(5668), pp.253-257. 
Endo, T. \& Yamano, K., 2010. Transport of proteins across or into the mitochondrial outer membrane. Biochimica et Biophysica acta, 1803(6), pp.706-714.

Finsterer, J., 2008. Leigh and Leigh-like syndrome in children and adults. Pediatric neurology, 39(4), pp.223-235.

Fontanesi, F. et al., 2006. Assembly of mitochondrial cytochrome c-oxidase, a complicated and highly regulated cellular process. American journal of physiology. Cell physiology, 291(6), pp.C1129-47.

Fontanesi, F. et al., 2010. Mss51 and Ssc1 facilitate translational regulation of cytochrome c oxidase biogenesis. Molecular and Cellular Biology, 30(1), pp.245-259.

Fontanesi, F., Clemente, P. \& Barrientos, A., 2011. Cox25 Teams Up with Mss51, Ssc1, and Cox14 to Regulate Mitochondrial Cytochrome c Oxidase Subunit 1 Expression and Assembly in Saccharomyces cerevisiae. Journal of Biological Chemistry, 286(1), pp.555566.

Fontanesi, F., 2013. Mechanisms of mitochondrial translational regulation. IUBMB Life, 65(5), pp.397-408.

Frazier, A.E. et al., 2006. Mdm38 interacts with ribosomes and is a component of the mitochondrial protein export machinery. The Journal of Cell Biology, 172(4), pp.553564.

Frey, T.G., Renken, C.W. \& Perkins, G.A., 2002. Insight into mitochondrial structure and function from electron tomography. Biochimica et Biophysica acta, 1555(1-3), pp.196203.

Gakh, O., Cavadini, P. \& Isaya, G., 2002. Mitochondrial processing peptidases. Biochimica et Biophysica acta, 1592(1), pp.63-77.

Gietz, R.D. \& Schiestl, R.H., 2007. Quick and easy yeast transformation using the LiAc/SS carrier DNA/PEG method. Nature Protocols, 2(1), pp.35-37.

Glerum, D.M. \& Tzagoloff, A., 1994. Isolation of a human cDNA for heme A:farnesyltransferase by functional complementation of a yeast cox10 mutant. Proceedings of the National Academy of Sciences of the United States of America, 91(18), pp.8452-8456.

Glerum, D.M., Koerner, T.J. \& Tzagoloff, A., 1995. Cloning and characterization of COX14, whose product is required for assembly of yeast cytochrome oxidase. The Journal of biological chemistry, 270(26), pp.15585-15590.

Glerum, D.M., Shtanko, A. \& Tzagoloff, A., 1996. Characterization of COX17, a yeast gene involved in copper metabolism and assembly of cytochrome oxidase. The Journal of biological chemistry, 271(24), pp.14504-14509.

Glerum, D.M. \& Tzagoloff, A., 1997. Submitochondrial distributions and stabilities of subunits 4,5 , and 6 of yeast cytochrome oxidase in assembly defective mutants. FEBS Letters, 412(3), pp.410-414.

Glerum, D.M. et al., 1997. COX15 codes for a mitochondrial protein essential for the assembly of yeast cytochrome oxidase. The Journal of biological chemistry, 272(30), pp.19088-19094. 
Golik, P. et al., 2003. The Rieske FeS protein encoded and synthesized within mitochondria complements a deficiency in the nuclear gene. Proceedings of the National Academy of Sciences of the United States of America, 100(15), pp.8844-8849.

Grandier-Vazeille, X. et al., 2001. Yeast mitochondrial dehydrogenases are associated in a supramolecular complex. Biochemistry, 40(33), pp.9758-9769.

Gray, M.W., Burger, G. \& Lang, B.F., 2001. The origin and early evolution of mitochondria. Genome Biology, 2(6), REVIEWS1018

Guzélin, E., Rep, M. \& Grivell, L.A., 1996. Afg3p, a mitochondrial ATP-dependent metalloprotease, is involved in degradation of mitochondrially-encoded Cox1, Cox3, Cob, Su6, Su8 and Su9 subunits of the inner membrane complexes III, IV and V. FEBS Letters, 381(1-2), pp.42-46.

Hackenbrock, C.R., Chazotte, B. \& Gupte, S.S., 1986. The random collision model and a critical assessment of diffusion and collision in mitochondrial electron transport. Journal of bioenergetics and biomembranes, 18(5), pp.331-368.

Hannappel, A., Bundschuh, F.A. \& Ludwig, B., 2011. Characterization of heme-binding properties of Paracoccus denitrificans Surf1 proteins. FEBS Journal, 278(10), pp.1769-1778.

Hannappel, A., Bundschuh, F.A. \& Ludwig, B., 2012. Role of Surf1 in heme recruitment for bacterial COX biogenesis. Biochimica et Biophysica Acta - Bioenergetics, 1817(6), pp.928-937.

He, S. \& Fox, T.D., 1997. Membrane translocation of mitochondrially coded Cox2p: distinct requirements for export of $\mathrm{N}$ and $\mathrm{C}$ termini and dependence on the conserved protein Oxa1p. Molecular biology of the cell, 8(8), pp.1449-1460.

Hederstedt, L., Lewin, A. \& Throne-Holst, M., 2005. Heme A Synthase Enzyme Functions Dissected by Mutagenesis of Bacillus subtilis CtaA. Journal of Bacteriology, 187(24), pp.8361-8369.

Heijne, von, G., 1986. Mitochondrial targeting sequences may form amphiphilic helices. The EMBO Journal, 5(6), pp.1335-1342.

Hell, K. et al., 1998. Oxa1p, an essential component of the N-tail protein export machinery in mitochondria. Proceedings of the National Academy of Sciences of the United States of America, 95(5), pp.2250-2255.

Hell, K., Neupert, W. \& Stuart, R.A., 2001. Oxa1p acts as a general membrane insertion machinery for proteins encoded by mitochondrial DNA. The EMBO Journal, 20(6), pp.1281-1288.

Hill, K. et al., 1998. Tom40 forms the hydrophilic channel of the mitochondrial import pore for preproteins. Nature, 395(6701), pp.516-521.

Hoch, F.L., 1992. Cardiolipins and biomembrane function. Biochimica et Biophysica acta, 1113(1), pp.71-133.

Hoppins, S., Lackner, L. \& Nunnari, J., 2007. The machines that divide and fuse mitochondria. Annual review of biochemistry, 76, pp.751-780. 
Horn, D. \& Barrientos, A., 2008. Mitochondrial copper metabolism and delivery to cytochrome c oxidase. IUBMB Life, 60(7), pp.421-429.

Horng, Y.-C. et al., 2004. Specific copper transfer from the Cox17 metallochaperone to both Sco1 and Cox11 in the assembly of yeast cytochrome $\mathrm{C}$ oxidase. The Journal of biological chemistry, 279(34), pp.35334-35340.

Hosler, J.P., Ferguson-Miller, S. \& Mills, D.A., 2006. Energy transduction: proton transfer through the respiratory complexes. Annual review of biochemistry, 75, pp.165-187.

Hutu, D.P. et al., 2008. Mitochondrial protein import motor: differential role of Tim44 in the recruitment of Pam 17 and J-complex to the presequence translocase. Molecular biology of the cell, 19(6), pp.2642-2649.

Ito, H. et al., 1983. Transformation of intact yeast cells treated with alkali cations. Journal of Bacteriology, 153(1), pp.163-168.

Janke, C. et al., 2004. A versatile toolbox for PCR-based tagging of yeast genes: new fluorescent proteins, more markers and promoter substitution cassettes. Yeast, 21(11), pp.947-962.

Jia, L. et al., 2003. Yeast Oxa1 interacts with mitochondrial ribosomes: the importance of the C-terminal region of Oxa1. The EMBO Journal, 22(24), pp.6438-6447.

Jia, L., Dienhart, M.K. \& Stuart, R.A., 2007. Oxa1 directly interacts with Atp9 and mediates its assembly into the mitochondrial F1Fo-ATP synthase complex. Molecular biology of the cell, 18(5), pp.1897-1908.

Jia, L., Kaur, J. \& Stuart, R.A., 2009. Mapping of the Saccharomyces cerevisiae Oxa1mitochondrial ribosome interface and identification of MrpL40, a ribosomal protein in close proximity to Oxa1 and critical for oxidative phosphorylation complex assembly. Eukaryotic cell, 8(11), pp.1792-1802.

Keil, M. et al., 2012. Oxa1-ribosome complexes coordinate the assembly of cytochrome C oxidase in mitochondria. Journal of Biological Chemistry, 287(41), pp.34484-34493.

Khalimonchuk, O., Ostermann, K. \& Rödel, G., 2005. Evidence for the association of yeast mitochondrial ribosomes with $\operatorname{Cox} 11 \mathrm{p}$, a protein required for the $\mathrm{Cu}(\mathrm{B})$ site formation of cytochrome c oxidase. Current genetics, 47(4), pp.223-233.

Khalimonchuk, O., Bird, A. \& Winge, D.R., 2007. Evidence for a pro-oxidant intermediate in the assembly of cytochrome oxidase. The Journal of biological chemistry, 282(24), pp.17442-17449.

Khalimonchuk, O. et al., 2010. Formation of the Redox Cofactor Centers during Cox1 Maturation in Yeast Cytochrome Oxidase. Molecular and Cellular Biology, 30(4), pp.1004-1017.

Khalimonchuk, O. et al., 2012. Oligomerization of heme o synthase in cytochrome oxidase biogenesis is mediated by cytochrome oxidase assembly factor Coa2. The Journal of biological chemistry, 287(32), pp.26715-26726.

Kispal, G. et al., 2005. Biogenesis of cytosolic ribosomes requires the essential iron-sulphur protein Rli1p and mitochondria. The EMBO Journal, 24(3), pp.589-598. 
Knop, M. et al., 1999. Epitope Tagging of Yeast Genes using a PCR-based Strategy: More Tags and Improved Practical Routines. Yeast, 15, pp.963-972.

Kohler, R. et al., 2009. YidC and 0xa1 form dimeric insertion pores on the translating ribosome. Molecular cell, 34(3), pp.344-353.

Koppen, M. \& Langer, T., 2007. Protein degradation within mitochondria: versatile activities of AAA proteases and other peptidases. Critical reviews in biochemistry and molecular biology, 42(3), pp.221-242.

Krogan, N.J. et al., 2006. Global landscape of protein complexes in the yeast Saccharomyces cerevisiae. Nature, 440(7084), pp.637-643.

Kruszewska, A., Szczeniak, B. \& Clalsse, M., 1980. Recombinational Analysis of OXI1 Mutants and Preliminary Analysis of Their Translation Products in S. cerevisiae. Current Genetics, 2(1), pp.45-51.

Kulik, W. et al., 2008. Bloodspot assay using HPLC-tandem mass spectrometry for detection of Barth syndrome. Clinical chemistry, 54(2), pp.371-378.

Künkele, K.P. et al., 1998. The preprotein translocation channel of the outer membrane of mitochondria. Cell, 93(6), pp.1009-1019.

Laemmli, U.K., 1970. Cleavage of structural proteins during the assembly of the head of bacteriophage T4. Nature, 227(5259), pp.680-685.

Langer, T. et al., 2001. AAA proteases of mitochondria: quality control of membrane proteins and regulatory functions during mitochondrial biogenesis. Biochemical Society transactions, 29(4), pp.431-436.

Lapuente-Brun, E. et al., 2013. Supercomplex assembly determines electron flux in the mitochondrial electron transport chain. Science, 340(6140), pp.1567-1570.

Leigh, D., 1951. Subacute necrotizing encephalomyelopathy in an infant. Journal of neurology, neurosurgery, and psychiatry, 14(3), pp.216-221.

Lemaire, C. et al., 2004. A Yeast Mitochondrial Membrane Methyltransferase-like Protein Can Compensate for oxa1 Mutations. Journal of Biological Chemistry, 279(46), pp.47464-47472.

Lill, R., 2009. Function and biogenesis of iron-sulphur proteins. Nature, 460(7257), pp.831838.

Luirink, J., Samuelsson, T. \& de Gier, J.W., 2001. YidC/Oxa1p/Alb3: evolutionarily conserved mediators of membrane protein assembly. FEBS Letters, 501(1), pp.1-5.

Martinou, J.-C. \& Youle, R.J., 2011. Mitochondria in apoptosis: Bcl-2 family members and mitochondrial dynamics. Developmental cell, 21(1), pp.92-101.

Mashkevich, G. et al., 1997. SHY1, the yeast homolog of the mammalian SURF-1 gene, encodes a mitochondrial protein required for respiration. The Journal of biological chemistry, 272(22), pp.14356-14364.

Meisinger, C., Pfanner, N. \& Truscott, K.N., 2006. Isolation of yeast mitochondria. Methods in Molecular Biology, (313), pp.33-39. 
Merz, S. \& Westermann, B., 2009. Genome-wide deletion mutant analysis reveals genes required for respiratory growth, mitochondrial genome maintenance and mitochondrial protein synthesis in Saccharomyces cerevisiae. Genome Biology, 10(9), R95.

Mick, D.U. et al., 2007. Shy1 couples Cox1 translational regulation to cytochrome c oxidase assembly. The EMBO Journal, 26(20), pp.4347-4358.

Mick, D.U. et al., 2010. Coa3 and Cox14 are essential for negative feedback regulation of COX1 translation in mitochondria. The Journal of Cell Biology, 191(1), pp.141-154.

Mick, D.U., Fox, T.D. \& Rehling, P., 2011. Inventory control: cytochrome c oxidase assembly regulates mitochondrial translation. Nature Reviews Molecular Cell Biology, 12(1), pp.14-20.

Mick, D.U. et al., 2012. MITRAC links mitochondrial protein translocation to respiratorychain assembly and translational regulation. Cell, 151(7), pp.1528-1541.

Mitchell, P. \& Moyle, J., 1968. Proton translocation coupled to ATP hydrolysis in rat liver mitochondria. European journal of biochemistry / FEBS, 4(4), pp.530-539.

Model, K., Meisinger, C. \& Kühlbrandt, W., 2008. Cryo-electron microscopy structure of a yeast mitochondrial preprotein translocase. Journal of molecular biology, 383(5), pp.1049-1057.

Moraes, C.T., Diaz, F. \& Barrientos, A., 2004. Defects in the biosynthesis of mitochondrial heme $\mathrm{c}$ and heme a in yeast and mammals. Biochimica et Biophysica acta, 1659(2-3), pp.153-159.

Mossmann, D., Meisinger, C. \& Vögtle, F.N., 2012. Processing of mitochondrial presequences. Biochimica et Biophysica acta, 1819(9-10), pp.1098-1106.

Nargang, F.E. et al., 2002. The 0xa1 protein forms a homooligomeric complex and is an essential part of the mitochondrial export translocase in Neurospora crassa. The Journal of biological chemistry, 277(15), pp.12846-12853.

Nett, J.H. \& Trumpower, B.L., 1996. Dissociation of import of the Rieske iron-sulfur protein into Saccharomyces cerevisiae mitochondria from proteolytic processing of the presequence. The Journal of biological chemistry, 271(43), pp.26713-26716.

Netter, P. et al., 1982. The cytochrome oxidase subunit I split gene in Saccharomyces cerevisiae: genetic and physical studies of the mtDNA segment encompassing the "cytochrome b-homologous" intron. Molecular \& general genetics, 188(1), pp.51-59.

Neupert, W. \& Herrmann, J.M., 2007. Translocation of proteins into mitochondria. Annual review of biochemistry, 76, pp.723-749.

Nijtmans, L. et al., 2001. Shy1p occurs in a high molecular weight complex and is required for efficient assembly of cytochrome c oxidase in yeast. FEBS Letters, 498, pp.46-51.

Nikolov, M. et al., 2011. Chromatin affinity purification and quantitative mass spectrometry defining the interactome of histone modification patterns. Molecular \& cellular proteomics, 10(11), M110.005371. 
Nobrega, M.P., Nobrega, F.G. \& Tzagoloff, A., 1990. COX10 codes for a protein homologous to the ORF1 product of Paracoccus denitrificans and is required for the synthesis of yeast cytochrome oxidase. The Journal of biological chemistry, 265(24), pp.14220-14226.

Oquendo, C.E., 2004. Functional and genetic studies demonstrate that mutation in the C0X15 gene can cause Leigh syndrome. Journal of Medical Genetics, 41(7), pp.540-544.

Ott, M. et al., 2006. Mba1, a membrane-associated ribosome receptor in mitochondria. The EMBO Journal, 25(8), pp.1603-1610.

Pan, Y., 2011. Mitochondria, reactive oxygen species, and chronological aging: a message from yeast. Experimental gerontology, 46(11), pp.847-852.

Papadopoulou, L.C. et al., 1999. Fatal infantile cardioencephalomyopathy with COX deficiency and mutations in SCO2, a COX assembly gene. Nature Genetics, 23(3), pp.333337.

Paumard, P. et al., 2002. Two ATP synthases can be linked through subunits $i$ in the inner mitochondrial membrane of Saccharomyces cerevisiae. Biochemistry, 41(33), pp.10390-10396.

Perez-Martinez, X., ABroadley, S. \& DFox, T., 2003. Mss51p promotes mitochondrial Cox1p synthesis and interacts with newly synthesized Cox1p. The EMBO Journal, 22(21), pp.5951-5961.

Perez-Martinez, X. et al., 2009. Dual functions of Mss51 couple synthesis of Cox1 to assembly of cytochrome c oxidase in Saccharomyces cerevisiae mitochondria. Molecular biology of the cell, 20(20), pp.4371-4380.

Péquignot, M.O. et al., 2001. Mutations in the SURF1 gene associated with Leigh syndrome and cytochrome c oxidase deficiency. Human mutation, 17(5), pp.374-381.

Pierrel, F. et al., 2007. Coa1 links the Mss51 post-translational function to Cox1 cofactor insertion in cytochrome c oxidase assembly. The EMBO Journal, 26(20), pp.4335-4346.

Pierrel, F. et al., 2008. Coa2 is an assembly factor for yeast cytochrome c oxidase biogenesis that facilitates the maturation of Cox1. Molecular and Cellular Biology, 28(16), pp.49274939.

Premsler, T. et al., 2009. Recent advances in yeast organelle and membrane proteomics. Proteomics, 9(20), pp.4731-4743.

Preuss, M. et al., 2001. Mba1, a novel component of the mitochondrial protein export machinery of the yeast Saccharomyces cerevisiae. The Journal of Cell Biology, 153(5), pp.1085-1096.

Rahman, S. et al., 1996. Leigh syndrome: clinical features and biochemical and DNA abnormalities. Annals of neurology, 39(3), pp.343-351.

Rehling, P. et al., 2003. Protein insertion into the mitochondrial inner membrane by a twinpore translocase. Science, 299(5613), pp.1747-1751.

Reif, S. et al., 2005. Conserved mechanism of Oxa1 insertion into the mitochondrial inner membrane. Journal of molecular biology, 354(3), pp.520-528. 
Reinders, J. et al., 2006. Toward the complete yeast mitochondrial proteome: multidimensional separation techniques for mitochondrial proteomics. Journal of proteome research, 5(7), pp.1543-1554.

Reinhold, R. et al., 2011. Mimicking a SURF1 allele reveals uncoupling of cytochrome c oxidase assembly from translational regulation in yeast. Human Molecular Genetics, 20(12), pp.2379-2393.

Rimessi, A. et al., 2008. The versatility of mitochondrial calcium signals: from stimulation of cell metabolism to induction of cell death. Biochimica et Biophysica acta, 1777(7-8), pp.808-816.

Schaefer, A.M. et al., 2004. The epidemiology of mitochondrial disorders-past, present and future. Biochimica et Biophysica acta, 1659(2-3), pp.115-120.

Scharfe, C. et al., 2009. Mapping gene associations in human mitochondria using clinical disease phenotypes. PLoS computational biology, 5(4), p.e1000374.

Schägger, H. \& Jagow, von, G., 1991. Blue native electrophoresis for isolation of membrane protein complexes in enzymatically active form. Analytical Biochemistry, 199(2), pp.223-231.

Schägger, H. \& Pfeiffer, K., 2000. Supercomplexes in the respiratory chains of yeast and mammalian mitochondria. The EMBO Journal, 19(8), pp.1777-1783.

Schiestl, R.H. \& Gietz, R.D., 1989. High efficiency transformation of intact yeast cells using single stranded nucleic acids as a carrier. Current genetics, 16(5-6), pp.339-346.

Schon, E.A. \& Manfredi, G., 2003. Neuronal degeneration and mitochondrial dysfunction. Journal of Clinical Investigation, 111(3), pp.303-312.

Shevchenko, A. et al., 2006. In-gel digestion for mass spectrometric characterization of proteins and proteomes. Nature Protocols, 1(6), pp.2856-2860.

Shingú-Vázquez, M. et al., 2010. The carboxyl-terminal end of Cox1 is required for feedback assembly regulation of Cox1 synthesis in Saccharomyces cerevisiae mitochondria. Journal of Biological Chemistry, 285(45), pp.34382-34389.

Shoubridge, E.A., 2001. Cytochrome c oxidase deficiency. American journal of medical genetics, 106(1), pp.46-52.

Sicheritz-Pontén, T., Kurland, C.G. \& Andersson, S.G., 1998. A phylogenetic analysis of the cytochrome $\mathrm{b}$ and cytochrome $\mathrm{c}$ oxidase I genes supports an origin of mitochondria from within the Rickettsiaceae. Biochimica et Biophysica acta, 1365(3), pp.545-551.

Sickmann, A. et al., 2003. The proteome of Saccharomyces cerevisiae mitochondria. Proceedings of the National Academy of Sciences of the United States of America, 100(23), pp.13207-13212.

Sikorski, R.S. \& Hieter, P., 1989. A system of shuttle vectors and yeast host strains designed for efficient manipulation of DNA in Saccharomyces cerevisiae. Genetics, 122(1), pp.1927. 
Simon, M. \& Faye, G., 1984. Steps in processing of the mitochondrial cytochrome oxidase subunit I pre-mRNA affected by a nuclear mutation in yeast. Proceedings of the National Academy of Sciences of the United States of America, 81(1), pp.8-12.

Soto, I.C. et al., 2012. Biogenesis and assembly of eukaryotic cytochrome c oxidase catalytic core. Biochimica et Biophysica Acta - Bioenergetics, 1817(6), pp.883-897.

Stoldt, S. et al., 2012. The inner-mitochondrial distribution of Oxa1 depends on the growth conditions and on the availability of substrates. Molecular biology of the cell, 23(12), pp.2292-2301.

Strogolova, V. et al., 2012. Rcf1 and Rcf2, members of the hypoxia-induced gene 1 protein family, are critical components of the mitochondrial cytochrome bc1-cytochrome c oxidase supercomplex. Molecular and Cellular Biology, 32(8), pp.1363-1373.

Szklarczyk, R., Wanschers, B.F., Cuypers, T.D., et al., 2012a. Iterative orthology prediction uncovers new mitochondrial proteins and identifies C12orf62 as the human ortholog of COX14, a protein involved in the assembly of cytochrome c oxidase. Genome Biology, 13(2), R12.

Szklarczyk, R., Wanschers, B.F.J., Nijtmans, L.G., et al., 2012b. A mutation in the FAM36A gene, the human ortholog of COX20, impairs cytochrome c oxidase assembly and is associated with ataxia and muscle hypotonia. Human Molecular Genetics, 22(4), pp.656667

Taylor, A.B. et al., 2001. Crystal structures of mitochondrial processing peptidase reveal the mode for specific cleavage of import signal sequences. Structure, 9(7), pp.615-625.

Teixeira, P.F. \& Glaser, E., 2013. Processing peptidases in mitochondria and chloroplasts. Biochimica et Biophysica acta, 1833(2), pp.360-370.

Teraoka, M. et al., 1999. Two novel mutations of SURF1 in Leigh syndrome with cytochrome c oxidase deficiency. Human genetics, 105(6), pp.560-563.

Thakar, K. et al., 2013. Identification of CRM1-dependent Nuclear Export Cargos Using Quantitative Mass Spectrometry. Molecular \& cellular proteomics, 12(3), pp.664-678.

Thomas, B.J. \& Rothstein, R., 1989. Elevated recombination rates in transcriptionally active DNA. Cell, 56(4), pp.619-630.

Tiranti, V. et al., 1998. Mutations of SURF-1 in Leigh disease associated with cytochrome c oxidase deficiency. Am. J. Hum. Genet., 63(6), pp.1609-1621.

Towpik, J., 2005. Regulation of mitochondrial translation in yeast. Cellular \& molecular biology letters, 10(4), pp.571-594.

Tsukihara, T. et al., 1995. Structures of metal sites of oxidized bovine heart cytochrome c oxidase at 2.8 A. Science, 269(5227), pp.1069-1074.

Tsukihara, T. et al., 1996. The whole structure of the 13-subunit oxidized cytochrome c oxidase at 2.8 A. Science, 272(5265), pp.1136-1144.

Tzagoloff, A. et al., 1993. On the functions of the yeast COX10 and COX11 gene products. Biochemistry and molecular biology international, 31(3), pp.593-598. 
Valnot, I., Kleist-Retzow, von, J.C., et al., 2000a. A mutation in the human heme A:farnesyltransferase gene (COX10) causes cytochrome c oxidase deficiency. Human Molecular Genetics, 9(8), pp.1245-1249.

Valnot, I., Osmond, S., et al., 2000b. Mutations of the SCO1 gene in mitochondrial cytochrome c oxidase deficiency with neonatal-onset hepatic failure and encephalopathy. American journal of medical genetics, 67(5), pp.1104-1109.

Van Der Laan, M., Rissler, M. \& Rehling, P., 2006. Mitochondrial preprotein translocases as dynamic molecular machines. FEMS yeast research, 6(6), pp.849-861.

Van Der Laan, M., Hutu, D.P. \& Rehling, P., 2010. On the mechanism of preprotein import by the mitochondrial presequence translocase. Biochimica et Biophysica acta, 1803(6), pp.732-739.

Vögtle, F.N. et al., 2009. Global analysis of the mitochondrial N-proteome identifies a processing peptidase critical for protein stability. Cell, 139(2), pp.428-439.

Vreken, P. et al., 2000. Defective remodeling of cardiolipin and phosphatidylglycerol in Barth syndrome. Biochemical and biophysical research communications, 279(2), pp.378382.

Vukotic, M. et al., 2012. Rcf1 Mediates Cytochrome Oxidase Assembly and Respirasome Formation, Revealing Heterogeneity of the Enzyme Complex. Cell Metabolism, 15(3), pp.336-47

Wallace, D.C. et al., 1988. Mitochondrial DNA mutation associated with Leber's hereditary optic neuropathy. Science, 242(4884), pp.1427-1430.

Wallace, D.C., 2007. Why do we still have a maternally inherited mitochondrial DNA? Insights from evolutionary medicine. Annual review of biochemistry, 76, pp.781-821.

Wang, P. \& Dalbey, R.E., 2011. Inserting membrane proteins: the YidC/Oxa1/Alb3 machinery in bacteria, mitochondria, and chloroplasts. Biochimica et Biophysica acta, 1808(3), pp.866-875.

Weraarpachai, W. et al., 2009. Mutation in TACO1, encoding a translational activator of COX $\mathrm{I}$, results in cytochrome c oxidase deficiency and late-onset Leigh syndrome. Nature Genetics, 41(7), pp.833-837.

Weraarpachai, W. et al., 2012. Mutations in C12orf62, a factor that couples COX I synthesis with cytochrome c oxidase assembly, cause fatal neonatal lactic acidosis. The American Journal of Human Genetics, 90(1), pp.142-151.

Wiedemann, N., Pfanner, N. \& Rehling, P., 2006. Import of precursor proteins into isolated yeast mitochondria. Methods in molecular biology, 313, pp.373-383.

Wittig, I., Braun, H.-P. \& Schägger, H., 2006. Blue native PAGE. Nature Protocols, 1(1), pp.418-428.

Yaffe, M.P. \& Schatz, G., 1984. Two nuclear mutations that block mitochondrial protein import in yeast. Proceedings of the National Academy of Sciences of the United States of America, 81(15), pp.4819-4823. 
Yoshida, M., Muneyuki, E. \& Hisabori, T., 2001. ATP synthase-a marvellous rotary engine of the cell. Nature Reviews Molecular Cell Biology, 2(9), pp.669-677.

Yoshikawa, S., Muramoto, K. \& Shinzawa-Itoh, K., 2011. Proton-pumping mechanism of cytochrome C oxidase. Annual review of biophysics, 40, pp.205-223.

Young, J.C., Barral, J.M. \& Ulrich Hartl, F., 2003. More than folding: localized functions of cytosolic chaperones. Trends in biochemical sciences, 28(10), pp.541-547.

Zambrano, A. et al., 2007. Aberrant translation of cytochrome c oxidase subunit 1 mRNA species in the absence of Mss51p in the yeast Saccharomyces cerevisiae. Molecular biology of the cell, 18(2), pp.523-535.

Zhang, M., Mileykovskaya, E. \& Dowhan, W., 2002. Gluing the respiratory chain together. Cardiolipin is required for supercomplex formation in the inner mitochondrial membrane. The Journal of biological chemistry, 277(46), pp.43553-43556.

Zhang, Y. et al., 2007. Clinical and molecular survey in 124 Chinese patients with Leigh or Leigh-like syndrome. Journal of Inherited Metabolic Disease, 30(2), p.265.

Zhu, Z. et al., 1998. SURF1, encoding a factor involved in the biogenesis of cytochrome c oxidase, is mutated in Leigh syndrome. Nature Genetics, 20(4), pp.337-343.

Zick, M., Rabl, R. \& Reichert, A.S., 2009. Cristae formation-linking ultrastructure and function of mitochondria. Biochimica et Biophysica acta, 1793(1), pp.5-19. 


\section{ACKNOWLEDGMENTS}

First and foremost, I would like to thank my supervisor Prof. Dr. Peter Rehling for giving me the opportunity to work on this interesting project. I would like to express my gratitude for his full support, for his guidance and wise advice throughout the past years.

I am indebted to my thesis committee members, Prof. Stefan Jakobs and Prof. Henning Urlaub, for helpful discussions and suggestions during our meetings.

I also have to thank Dr. Miroslav Nikolov, Monika Raabe and Uwe Pleßmann from the lab of Prof. Urlaub for performing all mass spec analyses of my isolations.

I am especially grateful to Dr. David Mick and Dr. Sven Dennerlein for all their help, advices and discussions and for being always ready to answer my questions.

Moreover, I would like to thank all coauthors who contributed to the publication of our results in Bareth et al. (2013)!

I am very grateful to all current and former colleagues in the department of Cellular Biochemistry (previously known as Biochemistry II) for always being helpful and providing a stimulating and friendly working atmosphere during all these years. Thank you all for your support, for discussions and tips but also for the fun during and after work. I want to especially thank David, Robert, Christian and Markus for introduction into many methods in the beginning of this project.

I want to thank the Göttingen Graduate School for Neurosciences, Biophysics und Molecular Biosciences (DFG Grants GSC 226/1, GSC 226/2) for financial support of this work. I would also like to thank the staff of the GGNB office as well as Petra Engelmann for administrative support.

I thank Christian Schulz, Sven Dennerlein, David Mick and Sylvie Callegari for critical reading and revision of this work.

I am grateful for the love and support of my family, not just during the period of my $\mathrm{PhD}$, but through all my life. I would also like to thank my friends in Göttingen, Balingen or wherever they are for their friendship throughout years and decades. And of course, I thank Hartmut for his love, his constant support and for making me smile! 